\title{
Nano Aluminium Nitride as a Solid Source of Ammonia for the Preparation of Hantzsch 1,4-Dihydropyridines and Bis-(1,4-dihydropyridines) in Water via One Pot Multicomponent Reaction
}

\author{
Arash Ghorbani-Choghamarani, ${ }^{, a}$ Mohammad Ali Zolfigol, ${ }^{b}$ Maryam Hajjami, ${ }^{b}$ Hamid \\ Goudarziafshar, ${ }^{a}$ Mohsen Nikoorazm, ${ }^{a}$ Somaieh Yousefi ${ }^{a}$ and Bahman Tahmasbi ${ }^{a}$ \\ ${ }^{a}$ Department of Chemistry, Faculty of Science, Ilam University, P.O. Box 69315516, Ilam, Iran \\ ${ }^{b}$ Faculty of Chemistry, Bu-Ali Sina University, P.O. Box 6517838683, Hamadan, Iran
}

\begin{abstract}
O trabalho mostra a atuação de nanopartículas de nitreto de alumínio em presença da água agindo como a fonte geradora de amônia, empregada na preparação de 1,4-diidropiridinas e bis(1,4-diidropiridinas). Um procedimento eficiente e simples, “one-pot", é apresentado para síntese de 1,4-diidropiridina e dos derivados de bis-(1,4-diidropiridina), obtidos pela reação do acetoacetato de metila ou acetoacetato de etila com os aldeídos ou dialdeídos e nitreto de alumínio em água a $80^{\circ} \mathrm{C}$, com elevada pureza e com bons rendimentos.
\end{abstract}

Nano aluminium nitride in the presence of water acts as solid source of ammonia, which is used for the preparation of 1,4-dihydropyridines and bis-(1,4-dihydropyridines). An efficient and simple procedure for the one-pot synthesis of 1,4-dihydropyridine and bis-(1,4-dihydropyridine) derivatives was achieved by combination of methyl acetoacetate or ethyl acetoacetate with aldehydes or dialdehydes and aluminium nitride at $80^{\circ} \mathrm{C}$ in water in high purity and good yields.

Keywords: 1,4-dihydropyridine, bis-(1,4-dihydropyridine), ethyl acetoacetate, methyl acetoacetate, nano aluminium nitride

\section{Introduction}

One of the most attractive synthetic strategies favored by organic chemists is the multi-component coupling reactions (MCRs), which allow the creation of several bonds in a single operation. ${ }^{1-6}$ Numerous heterocyclic compounds have the ability to mimic structures of peptides and to bind reversibly to proteins. ${ }^{7}$ An interesting example of useful scaffold is the 1,4-dihydropyridine (DHP) system, because of its ability to act as $\mathrm{NAD}(\mathrm{P}) \mathrm{H}$ analogue of 1,4-dihydronicotinamide. ${ }^{8}$ It is the most important class of calcium-channel modulators ${ }^{9,10}$ and has been introduced for the treatment of cardiovascular diseases such as nifedipine, nicradipine and amlodipine.

Recent studies have revealed that 1,4-dihydropyridines exhibit several other medicinal applications including neuroprotectant and platet anti-aggregation activity in addition to acting as cerebral antiischemic agents in the treatment of Alzheimer's disease and as a

*e-mail: arashghch58@yahoo.com chemosensitizer in tumor therapy. ${ }^{11-13}$ The classical method for the synthesis of these compounds is the Hantzsch reaction involving a multicomponent condensation of an aldehyde with a 1,3-dicarbonyl compound and $\mathrm{NH}_{3}{ }^{14}$ Recently, much effort has been devoted to developing more efficient methods for the synthesis of 1,4-dihydropyridines. ${ }^{15-20}$

\section{Results and Discussion}

Recently we have reported a new procedure for the preparation of 1,4-dihydropyridines by one-pot threecomponent condensation of an aliphatic or aromatic aldehyde, methyl acetoacetate or ethyl propiolate, and $\mathrm{NH}_{4} \mathrm{OAc}$ under neat conditions (Scheme 1). ${ }^{19}$

In continuation of this investigation we decided to disclose a novel synthetic protocol for the preparation of 1,4-dihydropyridine and bis-(1,4-dihydropyridine) derivatives by combination of nano aluminium nitride, aldehyde and methyl acetoacetate or ethyl acetoacetate in water as solvent at $80^{\circ} \mathrm{C}$. 


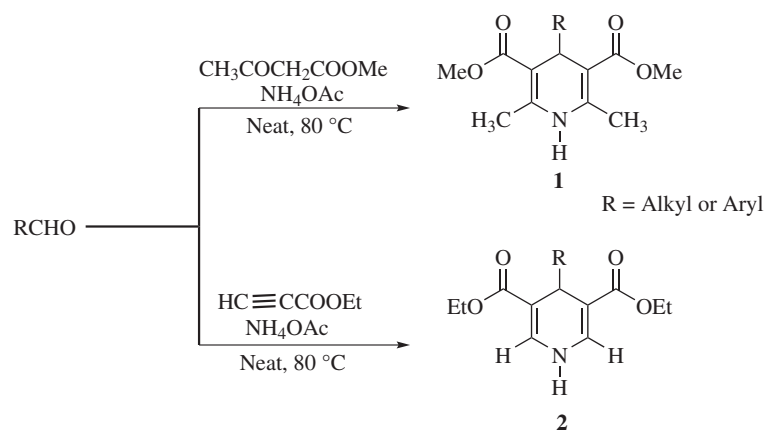

Scheme 1. Synthesis of 1,4-dihydropyridines with $\mathrm{NH}_{4} \mathrm{OAc}$ as source of ammonia

Aluminium nitride serves to generate a solution of ammonia via its reaction with water (equation 1), which is known reaction..$^{20-22}$

$\mathrm{AlN}+3 \mathrm{H}_{2} \mathrm{O} \rightarrow \mathrm{Al}(\mathrm{OH})_{3}+3 \mathrm{NH}_{3}$

Initially, to find optimal amount of nano aluminium nitride, 2,6-dimethyl-4-(4-chlorophenyl)-3,5dicarbomethoxy-1,4-dihydropyridine and 2,6-dimethyl4-(4-chlorophenyl)-3,5-dicarboethoxy-1,4-dihydropyridine were prepared via combination of 4-chlorobenzaldehyde $(1 \mathrm{mmol})$, methyl acetoacetate $(3 \mathrm{mmol})$ or ethyl acetoacetate $(3 \mathrm{mmol})$ and different amounts of nano aluminium nitride in water $(3 \mathrm{~mL})$ in a sealed tube at $80{ }^{\circ} \mathrm{C}$ (Scheme 2). The results of the preparation of 2,6-dimethyl-4-(4-chlorophenyl)-3,5-dicarbomethoxy1,4-dihydropyridine and 2,6-dimethyl-4-(4-chlorophenyl)3,5-dicarboethoxy-1,4-dihydropyridine as a function of the amounts of aluminium nitride are shown in Figure 1. The results showed $3 \mathrm{mmol}$ as the optimal amount of aluminium nitride, which was used in all reactions.

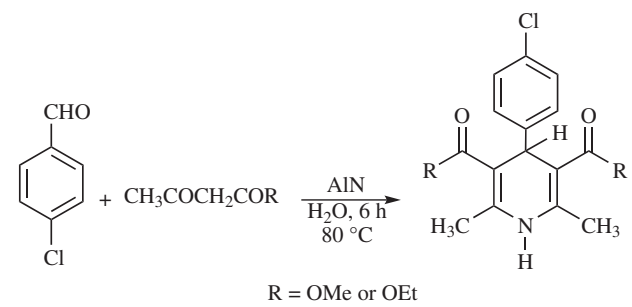

Scheme 2. Synthesis of 2,6-dimethyl-4-(4-chlorophenyl)-3,5dicarboalkoxy-1,4-dihydropyridines.

With optimal conditions in hand, a wide variety of 1,4-dihydropyridines were synthesized via combination of an aldehyde (or dialdehyde), alkyl acetoacetate and nano aluminium nitride, as a solid source of ammonia, in water at $80{ }^{\circ} \mathrm{C}$ (Scheme 3 and Table 1).

All of Hantzsch 1,4-dihydropyridines were prepared easily by mixing of the aldehyde, alkyl acetoacetate,

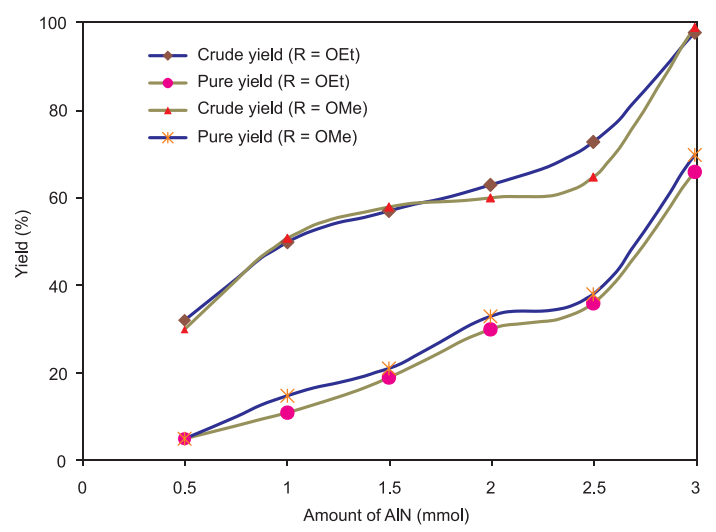

Figure 1. Optimization of the amount of aluminium nitride for the synthesis of 1,4-dihydropyridine derivatives.
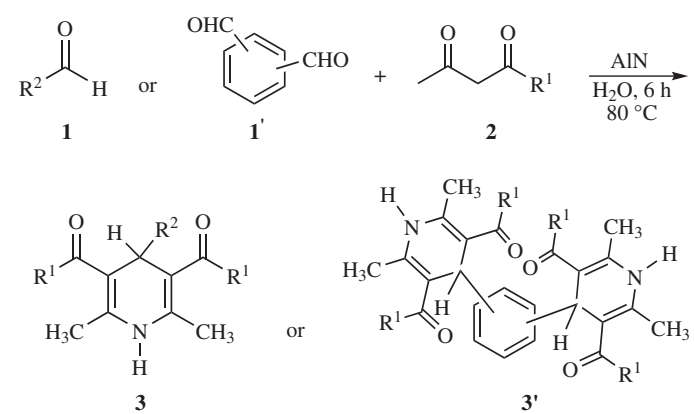

Scheme 3. Synthesis of 1,4-dihydropyridines using nano aluminium nitride as solid source.

aluminium nitride and water in a sealed tube at $80^{\circ} \mathrm{C}$. After $6 \mathrm{~h}$, sealed tube was cooled down to room temperature and the crude product extracted by dichloromethane. Dichloromethane was removed by evaporation. High pure 4-substituted 1,4-dihydro-2,6-dimethyl3,5-bis(alkoxycarbonyl)pyridines were obtained by recrystallization in the mixture of $\mathrm{H}_{2} \mathrm{O}$ and EtOH. Figure 2 shows scanning electron microscope (SEM) of aluminium nitride (a) and of the reaction mixture (b), after completion of the reaction of the transformation of entry 22 .

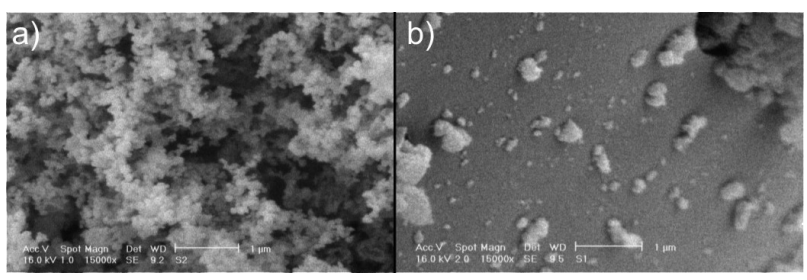

Figure 2. (a) SEM image of nano aluminium nitride; (b) SEM image of reaction mixture after reaction completion (entry 22).

To investigate the efficiency of this synthetic protocol in comparison with common reported procedures, where ammonium acetate was used instead of nano aluminium nitride, we prepared 2,6-dimethyl-4-(4-chlorophenyl)3,5-dicarbomethoxy-1,4-dihydropyridine by our new 
Table 1. Synthesis of 1,4-dihydropyridine derivatives via combination of aldehydes, alkyl acetoacetate and aluminium nitride at $80{ }^{\circ} \mathrm{C}$ in water

\begin{tabular}{|c|c|c|c|c|c|c|c|}
\hline entry & Compound & $\mathrm{R}^{1}$ & 1 or $\mathbf{1}^{\prime}$ & Product & Yield $A^{b}(B)^{c} \%$ & $\mathrm{mp}\left({ }^{\circ} \mathrm{C}\right)$ & Ref. \\
\hline 1 & $3 \mathbf{a}$ & $\mathrm{OMe}$ & $\left(\mathrm{CH}_{3}\right)_{2} \mathrm{CH}-\mathrm{CHO}$ & & $80(50)$ & $162.2-163.4$ & -- \\
\hline 2 & $3 \mathbf{b}$ & $\mathrm{OMe}$ & $\mathrm{CH}_{3}(\mathrm{CH})_{2}-\mathrm{CHO}$ & & $73(49)$ & $133-135$ & 19 \\
\hline 3 & $3 c$ & $\mathrm{OMe}$ & $\mathrm{C}_{6} \mathrm{H}_{5} \mathrm{CH}=\mathrm{CH}-\mathrm{CHO}$ & & $90(39)$ & $172-173.5$ & 17 \\
\hline 4 & 3d & $\mathrm{OMe}$ & $\mathrm{C}_{6} \mathrm{H}_{5}-\mathrm{CHO}$ & & $84(64)$ & $195.5-196.5$ & 19 \\
\hline 5 & $3 e$ & $\mathrm{OMe}$ & $4-\mathrm{Cl}-\mathrm{C}_{6} \mathrm{H}_{4}-\mathrm{CHO}$ & & $99(70)$ & 192.8-194 & -- \\
\hline 6 & $3 f$ & $\mathrm{OMe}$ & $4-\mathrm{Br}-\mathrm{C}_{6} \mathrm{H}_{4}-\mathrm{CHO}$ & & $80(61)$ & $197.8-198.9$ & 19 \\
\hline 7 & $3 g$ & $\mathrm{OMe}$ & $4-\mathrm{OCH}_{3}-\mathrm{C}_{6} \mathrm{H}_{4}-\mathrm{CHO}$ & & $84(62)$ & $187-187.6$ & 26 \\
\hline 8 & $3 \mathbf{h}$ & $\mathrm{OMe}$ & $4-\mathrm{F}-\mathrm{C}_{6} \mathrm{H}_{4}-\mathrm{CHO}$ & & $63(40)$ & $171-172$ & 25 \\
\hline 9 & $3 \mathbf{i}$ & $\mathrm{OMe}$ & $3,4-\left(\mathrm{OCH}_{3}\right)_{2}-\mathrm{C}_{6} \mathrm{H}_{3}-\mathrm{CHO}$ & & $92(63)$ & $148.5-149.5$ & 26 \\
\hline 10 & $3 \mathbf{j}$ & $\mathrm{OMe}$ & $3-\mathrm{NO}_{2}-\mathrm{C}_{6} \mathrm{H}_{4}-\mathrm{CHO}$ & & $99(60)$ & 204.1-205.9 & 19 \\
\hline
\end{tabular}


Table 1. Continuation

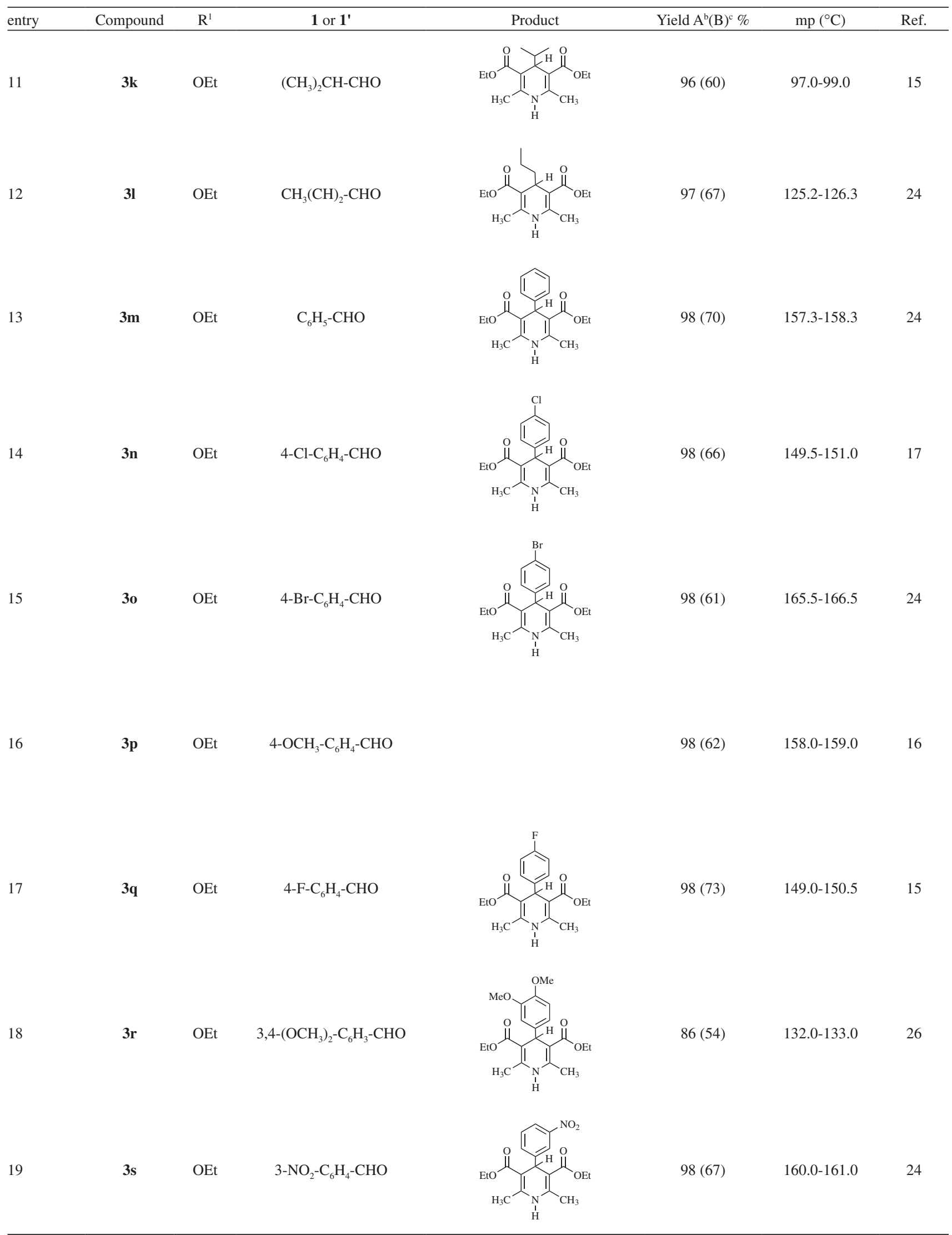


Table 1. Continuation

\begin{tabular}{|c|c|c|c|c|c|c|c|}
\hline entry & Compound & $\mathrm{R}^{1}$ & 1 or $1^{\prime}$ & Product & Yield $\mathrm{A}^{\mathrm{b}}(\mathrm{B})^{\mathrm{c}} \%$ & $\mathrm{mp}\left({ }^{\circ} \mathrm{C}\right)$ & Ref. \\
\hline 20 & 3'a & OEt & & & $86(75)$ & $181-185$ & -- \\
\hline 21 & 3'b & OEt & & & $63(48)$ & $289-293$ & -- \\
\hline 22 & $3 ' c$ & $\mathrm{OMe}$ & & & $87(71)$ & $118-121$ & -- \\
\hline 23 & 3'd & $\mathrm{OMe}$ & & & $75(51)$ & $175.0-179.0$ & -- \\
\hline
\end{tabular}

alkyl acetoacetate / aldehyde / aluminium nitride for entries 1-19 (3 mmol / $1 \mathrm{mmol} / 3 \mathrm{mmol})$; for entries 20-23 (6 mmol / $1 \mathrm{mmol} / 6 \mathrm{mmol}) .{ }^{\mathrm{b}} \mathrm{Crude}$ isolated yield. ${ }^{c}$ Yield of pure product after recrystallization in EtOH/ $\mathrm{H}_{2} \mathrm{O}$.

reported procedure (Scheme 4). ${ }^{19}$ As indicated in Scheme 4, 2,6-dimethyl-4-(4-chlorophenyl)-3,5-dicarbomethoxy-1,4dihydropyridine was obtained in 38\% yield (crude yield was $96 \%$ ). Consequently, this new procedure produces 1,4-dihydropyridines with high purity and yield in comparison with common procedures.<smiles>CC(=O)CC(=O)ONCc1ccc(Cl)cc1</smiles><smiles>COC(=O)C1=C(C)NC(C)=C(C(=O)OC)C1c1ccc(Cl)cc1</smiles>

Crude yield: $96 \%$ Pure isolated yield: $38 \%$

Scheme 4. Synthesis of 2,6-dimethyl-4-(4-chlorophenyl)-3,5dicarbomethoxy-1,4- dihydropyridine employing $\mathrm{NH}_{4} \mathrm{OAc}$.

\section{Conclusion}

In conclusion, we have demonstrated that the combination of different mono or di-aldehydes, alkyl acetoacetate and nano aluminium nitride in water allows a rapid and practical preparation of Hantzch 1,4-dihydropyridines. The reaction does not require the use of harsh conditions as well as harmful metal catalysts. Thus, this is an ecofriendly and environmentally friendly procedure for the synthesis of 1,4-dihydropyridine derivatives.

\section{Experimental}

\section{Apparatus}

${ }^{1} \mathrm{H}$ and ${ }^{13} \mathrm{C}$ NMR spectra were recorded on a Bruker 
DPX400 spectrometer $\left({ }^{1} \mathrm{H}\right.$ NMR, $400 \mathrm{MHz} ;{ }^{13} \mathrm{C}$ NMR, $100 \mathrm{MHz})$. Chemical shifts $(\delta)$ are reported in ppm and are referenced to the solvent, i.e., $7.26 / 77.1$ for $\mathrm{CDCl}_{3}$ and $2.49 / 39.5$ for DMSO- $d_{6}$. Multiplicities are described as br (broadened), s (singlet), $\mathrm{d}$ (doublet), $\mathrm{t}$ (triplet), $\mathrm{q}$ (quartet), $\mathrm{m}$ (multiplet). Coupling constants $(J)$ are reported in Hertz (Hz). IR spectra were measured with a Bomem (FT-IR) spectrometer. Thin layer chromatography (TLC) was performed on Merck Kieselgel 60 TLC plates. Purity and homogeneity of all materials was determined from TLC, ${ }^{1} \mathrm{H}$ NMR, and ${ }^{13} \mathrm{C}$ NMR.

Synthesis of 2,6-dimethyl-4-(3-nitrophenyl)-3,5dicarbomethoxy-1,4-dihydropyridine (3j), as a typical procedure

Aluminium nitride $(0.123 \mathrm{~g}, 3 \mathrm{mmol})$ was added to a stirring mixture of 3-nitrobenzaldehyde $(0.151 \mathrm{~g}$, $1 \mathrm{mmol})$ and methyl acetoacetate $(0.348 \mathrm{~g}, 3 \mathrm{mmol})$ at room temperature. The reaction vessel was sealed and allowed to warm to $80^{\circ} \mathrm{C}$ over $6 \mathrm{~h}$. Then reaction mixture cooled down to room temperature and the crude product extracted by dichloromethane. Dichloromethane was removed by simple evaporation. Finally crude product $(0.343 \mathrm{~g}, 99 \%)$ recrystallized from $\mathrm{EtOH} / \mathrm{H}_{2} \mathrm{O}$ to afford pure 2,6-dimethyl-4-(3-nitrophenyl)-3,5-dicarbomethoxy-1,4dihydropyridine in $60 \%$ yield $(0.207 \mathrm{~g})$ as yellow crystalline solid; mp: 204.1-205.9 ${ }^{\circ} \mathrm{C}$; IR (KBr) $v_{\max } / \mathrm{cm}^{-1}: 3349(\mathrm{~N}-\mathrm{H})$, $1704(\mathrm{C}=\mathrm{O}), 1650,1641,1529,1464,1377,1344,1219$; ${ }^{1} \mathrm{H}$ NMR $\left(400 \mathrm{MHz}, \mathrm{CDCl}_{3}\right) \delta 8.11(\mathrm{~s}, 1 \mathrm{H}), 8.00-8.02(\mathrm{~d}$, $1 \mathrm{H}, J 8.8 \mathrm{~Hz}), 7.63-7.65(\mathrm{~d}, 1 \mathrm{H}, J 8.0 \mathrm{~Hz}), 7.39-7.41(\mathrm{t}, 1 \mathrm{H}$, $J 8.0 \mathrm{~Hz}), 5.72(\mathrm{~s}, 1 \mathrm{H}), 5.11(\mathrm{~s}, 1 \mathrm{H}), 3.66(\mathrm{~s}, 6 \mathrm{H}), 2.38(\mathrm{~s}$, $6 \mathrm{H}) ;{ }^{13} \mathrm{C}$ NMR (100 MHz, $\left.\mathrm{CDCl}_{3}\right) \delta 167.5,149.6,148.4$, 145.0, 134.2, 128.7, 122.7, 121.4, 103.1, 52.9, 39.6, 19.6 .

\section{2,6-Dimethyl-4-(iso-propyl)-3,5-dicarbomethoxy-1,4-} dihydropyridine (3a)

Pale yellow crystalline solid; mp 162.2-163.4 ${ }^{\circ} \mathrm{C}$; IR $(\mathrm{KBr}) v_{\max } / \mathrm{cm}^{-1}: 3336(\mathrm{~N}-\mathrm{H}), 1706(\mathrm{C}=\mathrm{O}), 1652,1463$, 1435, 1377, 1219; ${ }^{1} \mathrm{H}$ NMR (400 MHz, $\left.\mathrm{CDCl}_{3}\right) \delta 5.64(\mathrm{~s}$, $1 \mathrm{H}), 3.90-3.89(\mathrm{~d}, 1 \mathrm{H}, J 5.2 \mathrm{~Hz}), 3.71(\mathrm{~s}, 6 \mathrm{H}), 2.31(\mathrm{~s}, 6 \mathrm{H})$, 1.58-1.62 (sep, 1H, J 6.8 Hz), 0.75-0.73 (d, 6H, J 6.8 Hz); ${ }^{13} \mathrm{C}$ NMR $\left(100 \mathrm{MHz}, \mathrm{CDCl}_{3}\right) \delta 169.2,145.1,101.3,50.8$, $38.8,35.4,19.2,18.2$.

2,6-Dimethyl-4-(4-chlorophenyl)-3,5-dicarbomethoxy-1,4dihydropyridine $(3 \boldsymbol{e})$

Pale yellow crystalline solid; mp $192.8-194.0^{\circ} \mathrm{C}$; IR $(\mathrm{KBr}) v_{\max } / \mathrm{cm}^{-1}: 3323(\mathrm{~N}-\mathrm{H}), 1702(\mathrm{C}=\mathrm{O}), 1650,1488$, $1465,1377,1219 ;{ }^{1} \mathrm{H}$ NMR $\left(400 \mathrm{MHz}, \mathrm{CDCl}_{3}\right) \delta$ 7.26-7.19 $(\mathrm{m}, 4 \mathrm{H}), 5.64(\mathrm{~s}, 1 \mathrm{H}), 4.98(\mathrm{~s}, 1 \mathrm{H}), 3.65(\mathrm{~s}, 6 \mathrm{H}), 2.35(\mathrm{~s}$,
$6 \mathrm{H}) ;{ }^{13} \mathrm{C} \mathrm{NMR}\left(100 \mathrm{MHz}, \mathrm{CDCl}_{3}\right) \delta 167.9,146.0,144.4$, 131.8, 129.1, 128.1, 103,6, 51.1, 39.0, 19.5.

1,3-Bis-(2,6-diethyl-3,5-dicarboethoxy-1,4-dihydropyridine) benzene (3'a)

Pale yellow crystalline solid; mp $181-185^{\circ} \mathrm{C} ;{ }^{1} \mathrm{H}$ NMR $\left(400 \mathrm{MHz}, \mathrm{DMSO}-d_{6}\right) \delta 8.75(\mathrm{~s}, 2 \mathrm{H}), 6.85-7.00(\mathrm{~m}, 4 \mathrm{H}), 4.79$ (s, 2H), 3.93-3.98 (q, 8H, J 6.8 Hz), 2.23 (s, 12H), 1.08-1.12 (t, $12 \mathrm{H}, J 6.8 \mathrm{~Hz}) ;{ }^{13} \mathrm{C}$ NMR $\left(100 \mathrm{MHz}\right.$, DMSO-d $\left.{ }_{6}\right) \delta 167.4$, 147.9, 145.7, 127.7, 126.7, 125.3, 102.3, 59.3, 18.6, 14.6.

\section{1,4-Bis-(2,6-diethyl-3,5-dicarboethoxy-1,4-dihydropyridine)} benzene (3'b)

Pale yellow crystalline solid; mp $289-293{ }^{\circ} \mathrm{C} ;{ }^{1} \mathrm{H}$ NMR $\left(400 \mathrm{MHz}, \mathrm{DMSO}-d_{6}\right) \delta 8.75(\mathrm{~s}, 2 \mathrm{H}), 6.95(\mathrm{~s}, 4 \mathrm{H}), 4.76$ (s, $2 \mathrm{H}), 3.93-4.00$ (q, 8H, J 7.2 Hz), 2.22 (s, 12H), 1.07-1.11 (t, $12 \mathrm{H}, J 7.2 \mathrm{~Hz}) ;{ }^{13} \mathrm{C}$ NMR (100 MHz, DMSO-d 6 ) $\delta 167.5$, 147.9, 146.1, 145.7, 127.3, 102.3, 59.4,18.7, 14.6.

1,3-Bis-(2,6-dimethyl-3,5-dicarboethoxy-1,4dihydropyridine) benzene $\left(\mathbf{3}^{\prime} \mathrm{c}\right)$

Pale yellow crystalline solid; mp $118-121^{\circ} \mathrm{C}$; ${ }^{1} \mathrm{H}$ NMR $\left(400 \mathrm{MHz}\right.$, DMSO- $\left.d_{6}\right) \delta 8.86(\mathrm{~s}, 2 \mathrm{H}), 6.84-7.01(\mathrm{~m}, 4 \mathrm{H}), 4.78$ (s, 2H), 3.51 (s, 12H), 2.24 (s, 12H); ${ }^{13} \mathrm{C}$ NMR $(100 \mathrm{MHz}$, DMSO-d $\left._{6}\right) \delta 167.9,147.8,146.1,128.1,126.3,125.0,102.0$, 51.0, 18.6.

1,4-Bis-(2,6-dimethyl-3,5-dicarboethoxy-1,4dihydropyridine) benzene $\left(\mathbf{3}^{\prime} \mathrm{d}\right)$

Pale yellow crystalline solid; mp $175-179^{\circ} \mathrm{C} ;{ }^{1} \mathrm{H}$ NMR $\left(400 \mathrm{MHz}, \mathrm{DMSO}-d_{6}\right) \delta 8.88(\mathrm{~s}, 2 \mathrm{H}), 6.93-7.12(\mathrm{~m}, 4 \mathrm{H}), 4.86$ (s, 2H), 3.54 (s, 12H), 2.24 (s, 12H); ${ }^{13} \mathrm{C}$ NMR (100 MHz, DMSO-d $\left._{6}\right) \delta 167.9,146.3,145.6,127.1,101.8,51.1,18.7$.

\section{Supplementary Information}

Supplementary data are available free of charge at http://jbcs.sbq.org.br, as PDF file.

\section{Acknowledgments}

The authors acknowledge Bu-Ali Sina University Research Council (Grant Number 32-1716), Center of Excellence in Development of Chemical Methods (CEDCM), National Foundation of Elites (BMN), and Ilam University, Ilam, Iran for financial support of this work.

\section{References}

1. Kantam, M. L.; Ramani, T.; Chakrapani, L.; Choudary, B. M.; Catal. Commun. 2009, 10, 370. 
2. Cui, S. L.; Wang, J.; Wang, Y. G.; Org. Lett. 2008, 10, 1267.

3. Li, L.; Zhang, G.; Zhu, A.; Zhang, L.; J. Org. Chem. 2008, 73, 3630 .

4. Ibrahim, M. A.; Abdel-Rahman, R. M.; Abdel-Halim, A. M.; Ibrahim, S. S.; Allimony H. A.; J. Braz. Chem. Soc. 2009, 20, 1275.

5. Navarrete-Encina, P. A.; Salazar, R.; Vega-Retter, C.; Perez, K.; Squella, J. A.; Nunez-Vergara, L. J.; J. Braz. Chem. Soc. 2010, $21,413$.

6. Suarez, M.; Molero, D.; Salfran, E.; Rodriguez, H.; Coro, J.; Saez, E.; Martinez-Alvarez, R.; Martin, N.; J. Braz. Chem. Soc. 2010 (in press).

7. Baskovc, J.; Bevk, D.; Stanovnik, B.; Svete, J.; J. Comb. Chem. 2009, 11, 500.

8. Zhu, X. Q.; Liu, Y.; Zhao, B. J.; Cheng, J. P.; J. Org. Chem. 2001, 66, 370.

9. Daryabari, N.; Akbarzadeh, T.; Amini, M.; Miri, R.; Mirkhani, H.; Shafiee, A.; J. Iran. Chem. Soc. 2007, 4, 30.

10. Shan, R.; Velazquez, C.; Knaus, E. E.; J. Med. Chem. 2004, 47, 254.

11. Suarez, M.; Verdecia, Y.; Illescas, B.; Martinez-Alvarez, R.; Avarez, A.; Ochoa, E.; Seoane, C.; Kayali, N.; Martin, N.; Tetrahedron 2003, 59, 9179.

12. Zarghi, A.; Sadeghi, H.; Fassihi, A.; Faizi, M.; Shafiee, A.; Il Farmaco 2003, 58, 1077.

13. Sabitha, G.; Reddy, G. S. K. K.; Reddy, C. S.; Yadav, J. S.; Tetrahedron Lett. 2003, 44, 4129.
14. Simon, C.; Constantieux, T.; Rodriguez, J.; Eur. J. Org. Chem. 2004, 4957

15. Sabitha, G.; Arundhathi, K.; Sudhakar, K.; Sastry, B. S.; Yadav, J. S.; Synth. Commun. 2009, 39, 2843.

16. Moghaddam, F. M.; Saeidian, H.; Mirjafary, Z.; Sadeghi, A.; J. Iran. Chem. Soc. 2009, 6, 317.

17. Rafiee, E.; Eavani, S.; Rashidzadeh, S.; Joshaghani, M.; Inorg. Chim. Acta 2009, 362, 3555.

18. Kumar, A.; Maurya, R. A.; Tetrahedron 2008, 64, 3477.

19. Ghorbani-Choghamarani, A.; Zolfigol, M. A.; Salehi, P.; Ghaemi, E.; Madrakian, E.; Nasr-Isfahanid, H.; Shahamirian, M.; Acta Chim. Slov. 2008, 55, 644.

20. Wu, L. Q.; Yang, C. G.; Yang, L. M.; Yang, L. J.; Heterocycles 2009, 78, 977.

21. Veitch, G. E.; Bridgwood, K. L.; Rands-Trevor, K.; Ley, S. V.; Synlett 2008, 2597.

22. Bridgwood, K. L.; Veitch, G. E.; Ley, S. V.; Org. Lett. 2008 , $10,3627$.

23. Wang, G. W.; Xia, J. J.; Miao, C. B.; Wu, X. L.; Bull. Chem. Soc. Jpn. 2006, 79, 454.

24. Zolfigol, M.A.; Safaiee, M.; Synlett 2004, 827.

25. Hernandez-Gallegos, Z.; Lehmann, P. A.; Hong, E.; Posadas, F.; Hernandez-Gallegos, E.; Eur. J. Med. Chem. 1995, 30, 355.

26. Sivamurugan, V.; Vinu, A.; Palanichamy, M.; Murugesan V.; Heteroat. Chem. 2006, 17, 267.

Submitted: August 18, 2010 Published online: November 4, 2010 


\title{
Nano Aluminium Nitride as a Solid Source of Ammonia for the Preparation of Hantzsch 1,4-Dihydropyridines and Bis-(1,4-dihydropyridines) in Water via One Pot Multicomponent Reaction
}

\author{
Arash Ghorbani-Choghamarani, ${ }^{*},{ }^{a}$ Mohammad Ali Zolfigol, ${ }^{b}$ Maryam Hajjami, ${ }^{b}$ Hamid \\ Goudarziafshar, ${ }^{a}$ Mohsen Nikoorazm, ${ }^{a}$ Somaieh Yousefi ${ }^{a}$ and Bahman Tahmasbi ${ }^{a}$ \\ ${ }^{a}$ Department of Chemistry, Faculty of Science, Ilam University, P.O. Box 69315516, Ilam, Iran \\ ${ }^{b}$ Faculty of Chemistry, Bu-Ali Sina University, P.O. Box 6517838683, Hamadan, Iran
}

\section{General methods}

${ }^{1} \mathrm{H}$ NMR and ${ }^{13} \mathrm{C}$ NMR spectra were recorded $400 \mathrm{MHz}$ $\left({ }^{1} \mathrm{H}\right)$ or $100.6 \mathrm{MHz}\left({ }^{13} \mathrm{C}\right)$. Chemical shifts are reported in $\operatorname{ppm}(\delta)$ and are referenced to the solvent, i.e., 7.26/77.1 for $\mathrm{CDCl}_{3}$ and 2.49/39.5 for DMSO- $d_{6}$. Multiplicities are described as br (broadened), s (singlet), $\mathrm{d}$ (doublet), $\mathrm{t}$ (triplet), q (quartet), m (multiplet). Coupling constants $(J)$ are reported in Hertz $(\mathrm{Hz})$. IR spectra were measured with a FT-IR spectrometer. Thin layer chromatography (TLC) was performed on Merck Kieselgel 60 TLC plates. Purity and homogeneity of all materials was determined from TLC, ${ }^{1} \mathrm{H}$ NMR, and ${ }^{13} \mathrm{C}$ NMR.

General procedure for the synthesis of 1,4-dihydropyridines

Aluminium nitride $(0.123 \mathrm{~g}, 3 \mathrm{mmol})$ was added to a stirring mixture of 3-nitrobenzaldehyde $(0.151 \mathrm{~g}$, $1 \mathrm{mmol})$ and methyl acetoacetate $(0.348 \mathrm{~g}, 3 \mathrm{mmol})$ at room temperature. The reaction vessel was sealed and allowed to warm to $80^{\circ} \mathrm{C}$ over $6 \mathrm{~h}$. Then reaction mixture cooled down to room temperature and the crude product extracted by dichloromethane. Dichloromethane was removed by simple evaporation. Finally crude product $(0.343 \mathrm{~g}, 99 \%)$ recrystallized from $\mathrm{EtOH} / \mathrm{H}_{2} \mathrm{O}$ to afford pure 1,4- dihydropyridine.

Synthesis of 2,6-dimethyl-4-(3-nitrophenyl)-3,5dicarbomethoxy-1,4- dihydropyridine (3j): as a typical procedure

Aluminium nitride $(0.123 \mathrm{~g}, 3 \mathrm{mmol})$ was added to the stirring mixture of 3- nitrobenzaldehyde $(0.151 \mathrm{~g}$, $1 \mathrm{mmol})$, methyl acetoacetate $(0.348 \mathrm{~g}, 3 \mathrm{mmol})$ at room temperature. The reaction vessel was sealed and allowed to warm to $80^{\circ} \mathrm{C}$ over $6 \mathrm{~h}$. Then reaction mixture cooled down to room temperature and the crude product extracted by dichloromethane. Dichloromethane was removed by simple evaporation. Finally crude product $(0.343 \mathrm{~g}$, 99\%) recrystallized from EtOH/ $\mathrm{H}_{2} \mathrm{O}$ to afford pure 2,6-dimethyl-4-(3-nitrophenyl)-3,5-dicarbomethoxy1,4-dihydropyridine in $60 \%$ yield $(0.207 \mathrm{~g})$ as yellow crystalline solid; mp 204.1-205.9 ${ }^{\circ} \mathrm{C}$; IR (KBr) $v_{\max }$ ' $\mathrm{cm}^{-1}: 3349(\mathrm{~N}-\mathrm{H}), 1704(\mathrm{C}=\mathrm{O}), 1650,1641,1529,1464$, 1377, 1344, 1219; ${ }^{1} \mathrm{H}$ NMR (400 MHz, $\mathrm{CDCl}_{3}$ ): $\delta 8.11$ $(\mathrm{s}, 1 \mathrm{H}), 8.00-8.02(\mathrm{~d}, 1 \mathrm{H}, J 8.8 \mathrm{~Hz}), 7.63-7.65(\mathrm{~d}, 1 \mathrm{H}, J$ $8.0 \mathrm{~Hz}), 7.39-7.41(\mathrm{t}, 1 \mathrm{H}, J 8.0 \mathrm{~Hz}), 5.72(\mathrm{~s}, 1 \mathrm{H}), 5.11$ (s, 1H), 3.66 (s, 6H), 2.38 (s, 6H) ppm; ${ }^{13} \mathrm{C}$ NMR $(100$ $\left.\mathrm{MHz}, \mathrm{CDCl}_{3}\right): \delta 167.5,149.6,148.4,145.0,134.2,128.7$, 122.7, 121.4, 103.1, 52.9, 39.6, $19.6 \mathrm{ppm}$.

2,6-Dimethyl-4-(iso-propyl)-3,5-dicarbomethoxy-1,4dihydropyridine (3a)

Pale yellow crystalline solid; $\mathrm{mp} 162.2-163.4^{\circ} \mathrm{C}$; IR $(\mathrm{KBr}) v_{\text {max }} / \mathrm{cm}^{-1}: 3336(\mathrm{~N}-\mathrm{H}), 1706(\mathrm{C}=\mathrm{O}), 1652,1463$, $1435,1377,1219 ;{ }^{1} \mathrm{H}$ NMR (400 MHz, $\left.\mathrm{CDCl}_{3}\right): \delta 5.64$ (s, 1H), 3.90-3.89 (d, 1H, J 5.2 Hz), $3.71(\mathrm{~s}, 6 \mathrm{H}), 2.31$ (s, 6H), 1.58-1.62 (sep, 1H, J 6.8 Hz), 0.75-0.73 (d, 6H, $J 6.8 \mathrm{~Hz}) \mathrm{ppm} ;{ }^{13} \mathrm{C}$ NMR $\left(100 \mathrm{MHz}, \mathrm{CDCl}_{3}\right): \delta 169.2$, $145.1,101.3,50.8,38.8,35.4,19.2,18.2 \mathrm{ppm}$.

2,6-Dimethyl-4-(n-propyl)-3,5-dicarbomethoxy-1,4dihydropyridine $(\mathbf{3 b})$

Pale yellow crystalline solid; mp $133-135^{\circ} \mathrm{C}$; IR $(\mathrm{KBr}) v_{\text {max }} / \mathrm{cm}^{-1}: 3343(\mathrm{~N}-\mathrm{H}), 1703(\mathrm{C}=\mathrm{O}), 1650,1492$, $1435,1329,1218 ;{ }^{1} \mathrm{H}$ NMR (400 MHz, $\left.\mathrm{CDCl}_{3}\right): \delta 5.54$ $(\mathrm{s}, 1 \mathrm{H}), 3.92(\mathrm{~m}, 1 \mathrm{H}), 3.72(\mathrm{~s}, 6 \mathrm{H}), 2.29(\mathrm{~s}, 6 \mathrm{H}), 1.28$ $1.20(\mathrm{~m}, 4 \mathrm{H}), 0.85-0.83(\mathrm{t}, 3 \mathrm{H}, J 6.8 \mathrm{~Hz}) \mathrm{ppm} ;{ }^{13} \mathrm{C} \mathrm{NMR}$ $\left(100 \mathrm{MHz}, \mathrm{CDCl}_{3}\right): \delta 168.6,145.0,103,50.9,39.2,32.8$, $19.4,17.9,14.3 \mathrm{ppm}$.

*e-mail: arashghch58@yahoo.com 
2,6-Dimethyl-4-(2-phenylethylene)-3,5-dicarbomethoxy-

1,4-dihydropyridine (3c)

Pale yellow crystalline solid; mp $172-173.5^{\circ} \mathrm{C}$; IR $(\mathrm{KBr}) \mathrm{v}_{\max } / \mathrm{cm}^{-1}: 3335(\mathrm{~N}-\mathrm{H}), 1698(\mathrm{C}=\mathrm{O}), 1647,1489$, $1465,1377,1226 ;{ }^{1} \mathrm{H} \mathrm{NMR}\left(400 \mathrm{MHz}, \mathrm{CDCl}_{3}\right): \delta 7.34-7.17$ $(\mathrm{m}, 5 \mathrm{H}), 6.20-6.17(\mathrm{~m}, 2 \mathrm{H}), 5.67(\mathrm{~s}, 1 \mathrm{H}), 4.63-4.62(\mathrm{~d}, 1 \mathrm{H}$, $J 5.2 \mathrm{~Hz}), 3.74$ (s, 6H), 2.35 (s, 6H) ppm; ${ }^{13} \mathrm{C}$ NMR $(100$ $\left.\mathrm{MHz}, \mathrm{CDCl}_{3}\right): \delta 168.0,145.4,137.7,131.8,128.4,128.0$, $126.9,126.3,101.2,51.2,36.2,19.4 \mathrm{ppm}$.

2,6-Dimethyl-4-(phenyl)-3,5-dicarbomethoxy-1,4dihydropyridine (3d)

Pale yellow crystalline solid; mp: $195.5-196.5^{\circ} \mathrm{C}$; IR $(\mathrm{KBr}) \mathrm{v}_{\max } / \mathrm{cm}^{-1}: 3342(\mathrm{~N}-\mathrm{H}), 1699(\mathrm{C}=\mathrm{O}), 1649,1464$, $1377,1344,1220 ;{ }^{1} \mathrm{H}$ NMR $\left(400 \mathrm{MHz}, \mathrm{CDCl}_{3}\right): \delta 7.26-$ $7.14(\mathrm{~m}, 5 \mathrm{H}), 5.64(\mathrm{~s}, 1 \mathrm{H}), 5.02(\mathrm{~s}, 1 \mathrm{H}), 3.66(\mathrm{~s}, 6 \mathrm{H}), 2.35$ (s, 6H) ppm; ${ }^{13} \mathrm{C} \mathrm{NMR} \mathrm{(100} \mathrm{MHz,} \mathrm{CDCl}_{3}$ ): $\delta$ 168.1, 147.4, 144.3, 128.0, 127.6, 126.2, 103.8, 51.0, 39.3, 19.5 ppm.

2,6-Dimethyl-4-(4-chlorophenyl)-3,5-dicarbomethoxy-1,4dihydropyridine $(\mathbf{3 e})$

Pale yellow crystalline solid; $\mathrm{mp} 192.8-194.0^{\circ} \mathrm{C}$; IR $(\mathrm{KBr}) v_{\max } / \mathrm{cm}^{-1}: 3323(\mathrm{~N}-\mathrm{H}), 1702(\mathrm{C}=\mathrm{O}), 1650,1488$, $1465,1377,1219 \mathrm{~cm}^{-1},{ }^{1} \mathrm{H} \mathrm{NMR}\left(400 \mathrm{MHz}, \mathrm{CDCl}_{3}\right): \delta 7.26-$ $7.19(\mathrm{~m}, 4 \mathrm{H}), 5.64(\mathrm{~s}, 1 \mathrm{H}), 4.98(\mathrm{~s}, 1 \mathrm{H}), 3.65(\mathrm{~s}, 6 \mathrm{H}), 2.35$ (s, 6H) ppm; ${ }^{13} \mathrm{C}$ NMR $\left(100 \mathrm{MHz}, \mathrm{CDCl}_{3}\right): \delta 167.9,146.0$, 144.4, 131.8, 129.1, 128.1, 103,6, 51.1, 39.0, 19.5 ppm.

2,6-Dimethyl-4-(4-bromophenyl)-3,5-dicarbomethoxy-1,4dihydropyridine ( $3 f$ )

Pale yellow crystalline solid; mp 197.8-198.9 ${ }^{\circ} \mathrm{C}$; IR $(\mathrm{KBr}) \mathrm{v}_{\max } / \mathrm{cm}^{-1}: 3319(\mathrm{~N}-\mathrm{H}), 1698(\mathrm{C}=\mathrm{O}), 1650,1483$, $1462,1435,1215$; ${ }^{1} \mathrm{H}$ NMR (400 MHz, $\mathrm{CDCl}_{3}$ ): $\delta 7.35-$ 7.33 (d, 2H, J 8.4), 7.16-7.14 (d, 2H, J 8.4), $5.71(\mathrm{~s}, 1 \mathrm{H})$, 4.97 (s, 1H), 3.65 (s, 6H), 2.34 (s, 6H) ppm; ${ }^{13} \mathrm{C}$ NMR (100 $\left.\mathrm{MHz}, \mathrm{CDCl}_{3}\right): \delta 167.9,146.5,144.4,131.1,129.5,120.0$, 103.5, 51.1, 39.0, $19.6 \mathrm{ppm}$.

2,6-Dimethyl-4-(4-methoxyphenyl)-3,5-dicarbomethoxy1,4-dihydropyridine (3g)

Pale yellow crystalline solid; mp 187.0-187.6 ${ }^{\circ} \mathrm{C}$; IR $(\mathrm{KBr}) v_{\text {max }} / \mathrm{cm}^{-1}: 3346(\mathrm{~N}-\mathrm{H}), 1697(\mathrm{C}=\mathrm{O}), 1649,1606$, 1465, 1377, 1302, 1216; ${ }^{1} \mathrm{H}$ NMR (400 MHz, $\left.\mathrm{CDCl}_{3}\right): \delta$ 7.20-7.18 (d, 2H, $J$ 8.8), 6.77-6.75 (d, 2H, $J$ 8.8), 5.58 (s, $1 \mathrm{H}), 4.95(\mathrm{~s}, 1 \mathrm{H}), 3.76(\mathrm{~s}, 3 \mathrm{H}), 3.66(\mathrm{~s}, 6 \mathrm{H}), 2.35(\mathrm{~s}, 6 \mathrm{H})$ $\mathrm{ppm} ;{ }^{13} \mathrm{C}$ NMR $\left(100 \mathrm{MHz}, \mathrm{CDCl}_{3}\right): \delta 168.1,158.0,143.9$, $139.9,128.6,113.4,104.2,55.1,51.0,38.4,19.6$ ppm.

2,6-Dimethyl-4-(4-flourophenyl)-3,5-dicarbomethoxy-1,4dihydropyridine (3h)

Pale yellow crystalline solid; mp 171.0-172.0 ${ }^{\circ} \mathrm{C}$; IR
$(\mathrm{KBr}) \mathrm{v}_{\max } / \mathrm{cm}^{-1}: 3349(\mathrm{~N}-\mathrm{H}), 1707(\mathrm{C}=\mathrm{O}), 1650,1465$, 1435, 1377, 1343, 1218; ${ }^{1} \mathrm{H}$ NMR (400 MHz, $\left.\mathrm{CDCl}_{3}\right): \delta$ 7.24-7.21 (m, 2H), 6.92-6.88 (m, 2H), $5.62(\mathrm{~s}, 1 \mathrm{H}), 4.99(\mathrm{~s}$, $1 \mathrm{H}), 3.66$ (s, 6H), 2.35 (s, 6H) ppm; ${ }^{13} \mathrm{C}$ NMR $(100 \mathrm{MHz}$, $\left.\mathrm{CDCl}_{3}\right): \delta 168.0,162.6,160.2,144.3,143.4,143.3,129.1$, 129, 114.8. 114.6, 103.8, 51.0, 38.7, $19.5 \mathrm{ppm}$.

2,6-Dimethyl-4-(3,4-dimethoxyphenyl)-3,5-dicarbomethoxy-1,4-dihydropyridine (3i)

Pale yellow crystalline solid; mp $148.5-149.5^{\circ} \mathrm{C}$; IR $(\mathrm{KBr}) \mathrm{v}_{\max } / \mathrm{cm}^{-1}: 3313(\mathrm{~N}-\mathrm{H}), 1698(\mathrm{C}=\mathrm{O}), 1649,1492$, 1463, 1377, 1267, 1213; ${ }^{1} \mathrm{H}$ NMR (400 MHz, $\mathrm{CDCl}_{3}$ ): $\delta$ $6.88(\mathrm{~s}, 1 \mathrm{H}), 6.79-6.72(\mathrm{q}, 2 \mathrm{H}, J 8 \mathrm{~Hz}), 5.62(\mathrm{~s}, 1 \mathrm{H}), 4.97$ (s, 1H), 3.85 (s, 3H), 3.83 (s 3H), 3.67 (s, 6H), 2.35 (s, $6 \mathrm{H}) \mathrm{ppm} ;{ }^{13} \mathrm{C}$ NMR $\left(100 \mathrm{MHz}, \mathrm{CDCl}_{3}\right): \delta 168.1,148.4$, 147.4, 144.0, 140.3, 119.4, 111.5, 111.0, 77.3, 55.8, 50.98, $38.8,19.6 \mathrm{ppm}$.

2,6-Dimethyl-4-(iso-propyl)-3,5-dicarboethoxy-1,4dihydropyridine $(\mathbf{3 k})$

Pale yellow crystalline solid; mp 97.0-99.0 ${ }^{\circ} \mathrm{C}$; IR $(\mathrm{KBr}) v_{\max } / \mathrm{cm}^{-1}: 3341(\mathrm{~N}-\mathrm{H}), 1696(\mathrm{C}=\mathrm{O}), 1652,1464$, 1377, 1216; ${ }^{1} \mathrm{H}$ NMR (400 MHz, $\left.\mathrm{CDCl}_{3}\right): \delta 5.55(\mathrm{~s}, 1 \mathrm{H})$, 4.25-4.12 (m, 4H), 3.94-3.92 (d, $1 \mathrm{H}, J 5.6 \mathrm{~Hz}), 2.31(\mathrm{~s}$, $6 \mathrm{H}), 1.32-1.29$ (t, 6H, J 7.2 Hz), 0.77-0.75 (d, 6H, J 6.8 $\mathrm{Hz}) \mathrm{ppm} ;{ }^{13} \mathrm{C} \mathrm{NMR}\left(100 \mathrm{MHz}, \mathrm{CDCl}_{3}\right): \delta 162.2,144.6$, $101.7,59.5,38.8,35.5,19.3,18.5,14.4 \mathrm{ppm}$.

2,6-Dimethyl-4-(n-propyl)-3,5-dicarboethoxy-1,4dihydropyridine (3l)

Pale yellow crystalline solid; $\mathrm{mp} 125.2-126.3^{\circ} \mathrm{C}$; IR $(\mathrm{KBr}) v_{\max } / \mathrm{cm}^{-1}: 3350(\mathrm{~N}-\mathrm{H}), 1699(\mathrm{C}=\mathrm{O}), 1465,1378$, 1299, $1213 ;{ }^{1} \mathrm{H}$ NMR (400 MHz, $\mathrm{CDCl}_{3}$ ): $\delta 5.49(\mathrm{~s}, 1 \mathrm{H})$, 4.25-4.12 (m, 4H), 3.96-3.93 (t, 1H, J 5.2 Hz), $2.29(\mathrm{~s}, 6 \mathrm{H})$, $1.32-1.29(\mathrm{t}, 6 \mathrm{H}, J 7.2 \mathrm{~Hz}), 1.25-1.23(\mathrm{~m}, 4 \mathrm{H}), 0.87-0.83(\mathrm{t}$, $3 \mathrm{H}, J 7.2 \mathrm{~Hz}) \mathrm{ppm} ;{ }^{13} \mathrm{C} \mathrm{NMR}\left(100 \mathrm{MHz}, \mathrm{CDCl}_{3}\right): \delta 168.2$, 144.7, 103.4, 59.6, 39.3, 32.7, 19.4, 18.0, 14.4, 14.3 ppm.

2,6-Dimethyl-4-(phenyl)-3,5-dicarboethoxy-1,4dihydropyridine (3m)

Pale yellow crystalline solid; mp $157.3-158.3{ }^{\circ} \mathrm{C}$; IR $(\mathrm{KBr}) \mathrm{v}_{\max } / \mathrm{cm}^{-1}: 3340(\mathrm{~N}-\mathrm{H}), 1687(\mathrm{C}=\mathrm{O}), 1650,1486$, 1464, 1374, 1247, 1211; ${ }^{1} \mathrm{H}$ NMR (400 MHz, $\mathrm{CDCl}_{3}$ ): $\delta$ 7.30-7.13 (m, 5H), $5.57(\mathrm{~s}, 1 \mathrm{H}), 5.00(\mathrm{~s}, 1 \mathrm{H}), 4.13-4.08$ (q, 4H, J 7.2 Hz), 2.34 (s, 6H), 1.25-1.21 (t, 6H, J 7.2 Hz) ppm; ${ }^{13} \mathrm{C}$ NMR (100 MHz, $\left.\mathrm{CDCl}_{3}\right): \delta 167.7,147.8,144.0$, 128.0, 127.8, 126.1, 104.0, 59.7, 39.6, 19.5, $14.3 \mathrm{ppm}$.

2,6-Dimethyl-4-(4-chlorophenyl)-3,5-dicarboethoxy-1,4dihydropyridine ( $3 \boldsymbol{n})$

Pale yellow crystalline solid; mp $149.5-151.0{ }^{\circ} \mathrm{C}$; IR 
$(\mathrm{KBr}) \mathrm{v}_{\max } / \mathrm{cm}^{-1}: 3357(\mathrm{~N}-\mathrm{H}), 1695(\mathrm{C}=\mathrm{O}), 1652,1486$, $1463,1377,1214 ;{ }^{1} \mathrm{H}$ NMR $\left(400 \mathrm{MHz}, \mathrm{CDCl}_{3}\right): \delta 7.23-$ $7.21(\mathrm{~d}, 2 \mathrm{H}, J 6.8 \mathrm{~Hz}), 7.18-7.16(\mathrm{~d}, 2 \mathrm{H}, J 8 \mathrm{~Hz}), 5.56(\mathrm{~s}$, $1 \mathrm{H}), 4.96(\mathrm{~s}, 1 \mathrm{H}), 4.10(\mathrm{~m}, 4 \mathrm{H}), 2.34(\mathrm{~s}, 6 \mathrm{H}), 1.24-1.21(\mathrm{t}$, $6 \mathrm{H}, J 6.4 \mathrm{~Hz}) \mathrm{ppm} ;{ }^{13} \mathrm{C} \mathrm{NMR}\left(100 \mathrm{MHz}, \mathrm{CDCl}_{3}\right): \delta 167.5$, $146.4,144.1,131.7,129.4,127.9,103.8,59.8,39.3,19.5$, $14.3 \mathrm{ppm}$.

2,6-Dimethyl-4-(4-bromophenyl)-3,5-dicarboethoxy-1,4dihydropyridine (3o)

Pale yellow crystalline solid; $\mathrm{mp} 165.5-166.5^{\circ} \mathrm{C}$; IR $(\mathrm{KBr}) v_{\max } / \mathrm{cm}^{-1}: 3360(\mathrm{~N}-\mathrm{H}), 1693(\mathrm{C}=\mathrm{O}), 1652,1486$, $1463,1377,1214 ;{ }^{1} \mathrm{H}$ NMR $\left(400 \mathrm{MHz}, \mathrm{CDCl}_{3}\right): \delta 7.34-$ $7.32(\mathrm{~d}, 2 \mathrm{H}, J 8.4 \mathrm{~Hz}), 7.18-7.15(\mathrm{~d}, 2 \mathrm{H}, J 8.4 \mathrm{~Hz}), 5.61$ (s, 1H), 4.95 (s, 1H), 4.11-4.08 (q, 4H, J 4.8 Hz), 2.33 (s, $6 \mathrm{H}), 1.25-1.21(\mathrm{t}, 6 \mathrm{H}, J 7.2 \mathrm{~Hz}) \mathrm{ppm} ;{ }^{13} \mathrm{C}$ NMR $(100 \mathrm{MHz}$, $\left.\mathrm{CDCl}_{3}\right): \delta 167.4,146.9,144.1,130.9,129.8,119.9,103.7$, $59.8,39.3,19.5,14.3 \mathrm{ppm}$.

2,6-Dimethyl-4-(4-methoxyphenyl)-3,5-dicarboethoxy-1,4dihydropyridine $(3 p)$

Pale yellow crystalline solid; mp $158.0-159.0^{\circ} \mathrm{C}$; IR $(\mathrm{KBr}) v_{\max } / \mathrm{cm}^{-1}: 3340(\mathrm{~N}-\mathrm{H}), 1689(\mathrm{C}=\mathrm{O}), 1649,1488$, 1464, 1373, 1301, 1211; ${ }^{1} \mathrm{H}$ NMR (400 MHz, $\left.\mathrm{CDCl}_{3}\right): \delta$ 7.22-7.19 (d, 2H, $J 8.8 \mathrm{~Hz}), 6.77-6.75(\mathrm{~d}, 2 \mathrm{H}, J 8.4 \mathrm{~Hz})$, $5.60(\mathrm{~s}, 1 \mathrm{H}), 4.94(\mathrm{~s}, 1 \mathrm{H}), 4.13-4.06(\mathrm{q}, 4 \mathrm{H}, J 5.2 \mathrm{~Hz}), 3.76$ (s, 3H), 2.33 (s, 6H), 1.25-1.22 (t, 6H, J $6.8 \mathrm{~Hz}) \mathrm{ppm} ;{ }^{13} \mathrm{C}$ NMR (100 MHz, $\left.\mathrm{CDCl}_{3}\right): \delta 167.8,143.7,140.4,128.9$, $113.2,104.3,59.7,55.1,38.8,19.5,14.3 \mathrm{ppm}$.

2,6-Dimethyl-4-(4-fluorophenyl)-3,5-dicarboethoxy-1,4dihydropyridine $(\mathbf{3 q})$

Pale yellow crystalline solid; mp $149.0-150.5^{\circ} \mathrm{C}$; IR $(\mathrm{KBr}) \mathrm{v}_{\max } / \mathrm{cm}^{-1}: 3342(\mathrm{~N}-\mathrm{H}), 1686(\mathrm{C}=\mathrm{O}), 1650,1488$, $1461,1373,1299,1211 ;{ }^{1} \mathrm{H}$ NMR (400 MHz, DMSO- $d_{6}$ ): $\delta 8.82(\mathrm{~s}, 1 \mathrm{H}), 7.16-7.00(\mathrm{~m}, 4 \mathrm{H}), 4.83(\mathrm{~s}, 1 \mathrm{H}), 3.99-3.96$ (q, 4H, J 7.2 Hz), $2.24(\mathrm{~s}, 6 \mathrm{H}), 1.13-1.10(\mathrm{t}, 6 \mathrm{H}, J 7.2 \mathrm{~Hz})$ ppm; ${ }^{13} \mathrm{C}$ NMR (100 MHz, DMSO- $\left.d_{6}\right): \delta 167.3,162.2$, $159.8,145.9,144.9,144.8,129.6,129.5,115.0,114.7$, $102.3,59.4,38.8,18.6,14.5 \mathrm{ppm}$.

2,6-Dimethyl-4-(3,4-dimethoxyphenyl)-3,5-dicarboethoxy1,4-dihydropyridine (3r)

Pale yellow crystalline solid; $\mathrm{mp} 132.0-133.0^{\circ} \mathrm{C}$; IR $(\mathrm{KBr}) v_{\max } / \mathrm{cm}^{-1}: 3352(\mathrm{~N}-\mathrm{H}), 1692(\mathrm{C}=\mathrm{O}), 1644,1623$, $1463,1377,1304,1122 ;{ }^{1} \mathrm{H}$ NMR (400 MHz, DMSO- $\left.d_{6}\right)$ : $\delta$ 6.89-6.72 (m, 3H), $5.57(\mathrm{~s}, 1 \mathrm{H}), 4.95(\mathrm{~s}, 1 \mathrm{H}), 4.15-4.08$ (m, 4H), $3.84(\mathrm{~s}, 3 \mathrm{H}), 3.83(\mathrm{~s}, 3 \mathrm{H}), 2.34(\mathrm{~s}, 6 \mathrm{H}), 1.26-1.23$ (t, $6 \mathrm{H}, J 6.8 \mathrm{~Hz}) \mathrm{ppm} ;{ }^{13} \mathrm{C}$ NMR $\left(100 \mathrm{MHz}\right.$, DMSO- $\left.d_{6}\right)$ : $\delta 167.7,148.1,147.3,143.8,140.8,119.8,111.8,110.9$, $104.1,59.7,55.8,55.7,39.0,19.5,14.3 \mathrm{ppm}$.
2,6-Dimethyl-4-(3-nitrophenyl)-3,5-dicarboethoxy-1,4dihydropyridine (3s)

Pale yellow crystalline solid; $\mathrm{mp} 160.0-161.0^{\circ} \mathrm{C}$; IR (KBr) $v_{\text {max }} / \mathrm{cm}^{-1}: 3342(\mathrm{~N}-\mathrm{H}), 1699(\mathrm{C}=\mathrm{O}), 1652,1464$, $1376,1348,1211 ;{ }^{1} \mathrm{H}$ NMR (400 MHz, DMSO- $\left.d_{6}\right): \delta 8.13$ (s, 1H), 8.02-8.00 (d, 1H, J 8.4 Hz), $5.68(\mathrm{~s}, 1 \mathrm{H}), 5.10(\mathrm{~s}$, $1 \mathrm{H}), 4.14-4.06$ (q, 4H, J 7.2 Hz), 2.38 (s, 6H), 1.25-1.21 (t, $6 \mathrm{H}, J 7.2 \mathrm{~Hz}) \mathrm{ppm} ;{ }^{13} \mathrm{C}$ NMR $\left(100 \mathrm{MHz}\right.$, DMSO- $\left.d_{6}\right)$ : $\delta 167.1,149.9,148.2,144.8,134.5,128.6,123.1,121.3$, 103.3, 60.0, 40.0, 19.6, $14.2 \mathrm{ppm}$.

1,3-Bis-(2,6-diethyl-3,5-dicarboethoxy-1,4-dihydropyridine) benzene (3'a)

Pale yellow crystalline solid; $\mathrm{mp} 181-185^{\circ} \mathrm{C}$; ${ }^{1} \mathrm{H}$ NMR (400 MHz, DMSO- $\left.d_{6}\right): \delta 8.75(\mathrm{~s}, 2 \mathrm{H}), 6.85-7.00(\mathrm{~m}, 4 \mathrm{H})$, 4.79 (s, 2H), 3.93-3.98 (q, 8H, J 6.8 Hz), 2.23 (s, 12H), $1.08-1.12(\mathrm{t}, 12 \mathrm{H}, J 6.8 \mathrm{~Hz}) \mathrm{ppm} ;{ }^{13} \mathrm{C}$ NMR $(100 \mathrm{MHz}$, DMSO- $\left.d_{6}\right): \delta 167.4,147.9,145.7,127.7,126.7,125.3$, 102.3, 59.3,18.6, $14.6 \mathrm{ppm}$.

1,4-Bis-(2,6-diethyl-3,5-dicarboethoxy-1,4-dihydropyridine) benzene (3'b)

Pale yellow crystalline solid; mp $289-293{ }^{\circ} \mathrm{C} ;{ }^{1} \mathrm{H}$ NMR (400 MHz, DMSO- $\left.d_{6}\right): \delta 8.75$ (s, 2H), $6.95(\mathrm{~s}, 4 \mathrm{H}), 4.76$ (s, 2H), 3.93-4.00 (q, 8H, J 7.2 Hz), 2.22 (s, 12H), 1.07-1.11 (t, $12 \mathrm{H}, J 7.2 \mathrm{~Hz})$ ppm. ${ }^{13} \mathrm{C}$ NMR $\left(100 \mathrm{MHz}\right.$, DMSO- $\left.d_{6}\right)$ : $\delta 167.5,147.9,146.1,145.7,127.3,102.3,59.4,18.7,14.6$ ppm.

1,3-Bis-(2,6-dimethyl-3,5-dicarboethoxy-1,4-dihydropyridine) benzene (3'c)

Pale yellow crystalline solid; mp $118-121{ }^{\circ} \mathrm{C} ;{ }^{1} \mathrm{H}$ NMR (400 MHz, DMSO- $\left.d_{6}\right): \delta 8.86(\mathrm{~s}, 2 \mathrm{H}), 6.84-7.01(\mathrm{~m}, 4 \mathrm{H})$, $4.78(\mathrm{~s}, 2 \mathrm{H}), 3.51(\mathrm{~s}, 12 \mathrm{H}), 2.24$ (s, 12H) ppm; ${ }^{13} \mathrm{C} \mathrm{NMR}$ (100 MHz, DMSO- $\left.d_{6}\right): \delta 167.9,147.8,146.1,128.1,126.3$, 125.0, 102.0, 51.0, $18.6 \mathrm{ppm}$.

1,4-Bis-(2,6-dimethyl-3,5-dicarboethoxy-1,4-dihydropyridine) benzene (3'd)

Pale yellow crystalline solid; mp $175-179^{\circ} \mathrm{C}$; ${ }^{1} \mathrm{H}$ NMR (400 MHz, DMSO- $\left.d_{6}\right): \delta 8.88(\mathrm{~s}, 2 \mathrm{H}), 6.93-7.12(\mathrm{~m}, 4 \mathrm{H})$, $4.86(\mathrm{~s}, 2 \mathrm{H}), 3.54(\mathrm{~s}, 12 \mathrm{H}), 2.24$ (s, 12H) ppm; ${ }^{13} \mathrm{C} \mathrm{NMR}$ (100 MHz, DMSO- $\left.d_{6}\right): \delta 167.9,146.3,145.6,127.1,101.8$, 51.1, 18.7 ppm. 


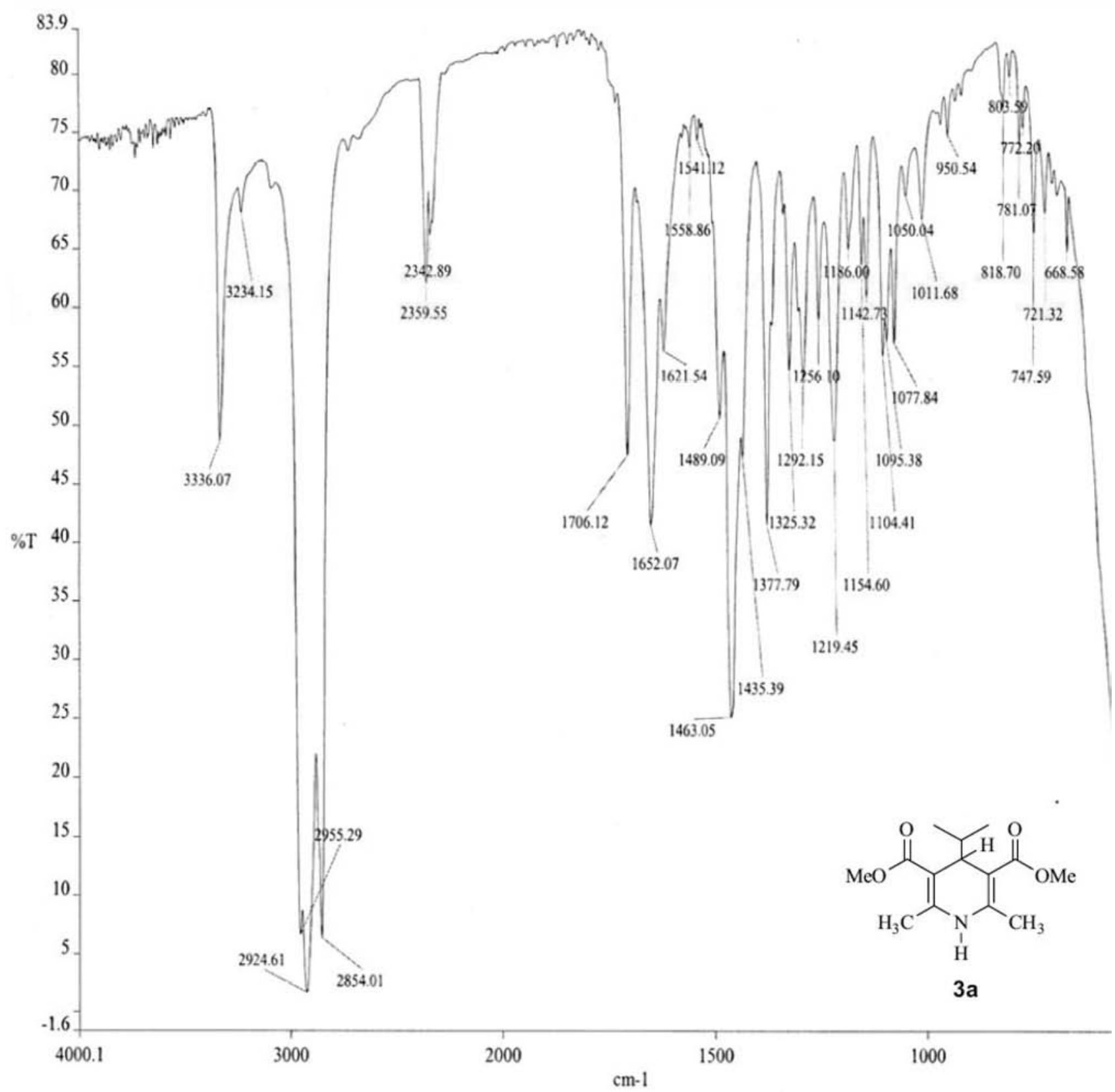

Figure S1. IR spectrum of 2,6-dimethyl-4-(iso-propyl)-3,5-dicarbomethoxy-1,4-dihydropyridine. 
<smiles>COC(=O)C1=C(C)NC(C)=C(C(=O)OC)C1(C)C(C)C</smiles>

$3 a$

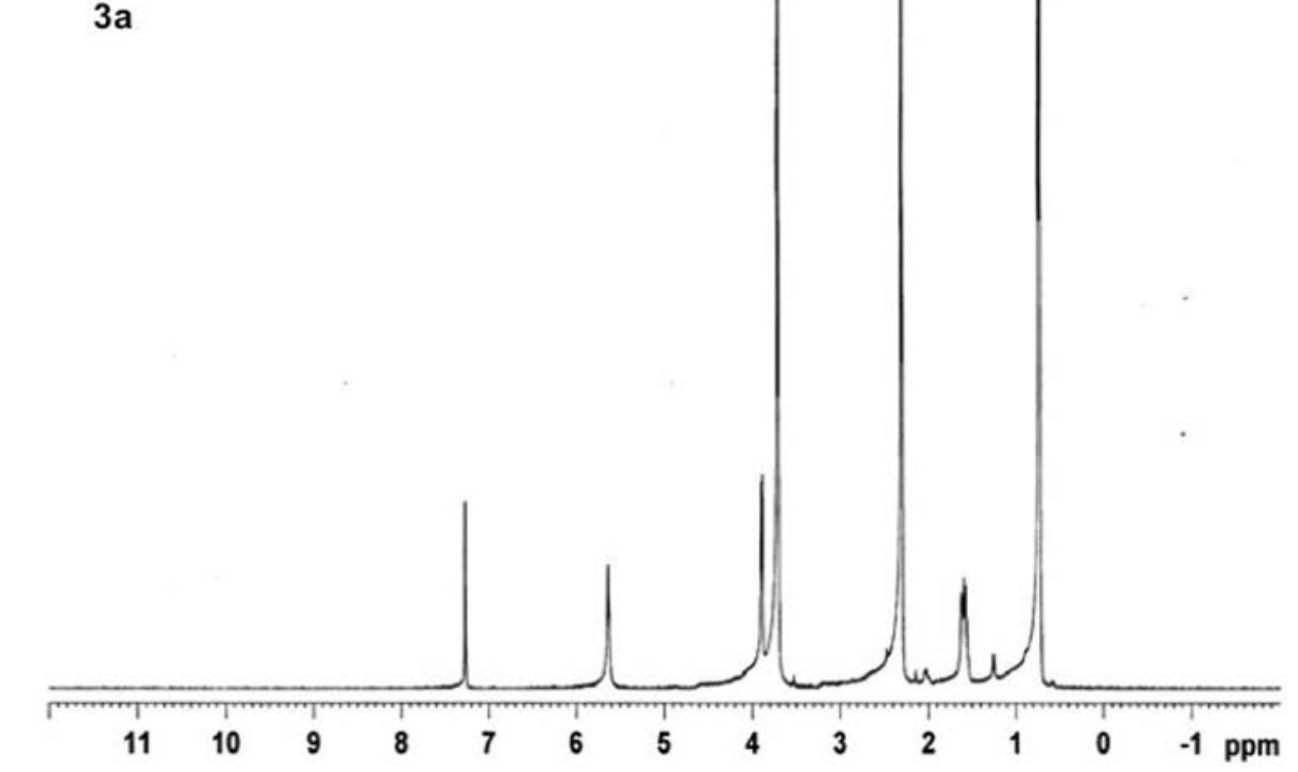

Figure S2. ${ }^{1} \mathrm{H}$ NMR spectrum (400 MHz, $\mathrm{CDCl}_{3}$ ) of 2,6-dimethyl-4-(iso-propyl)-3,5-dicarbomethoxy-1,4-dihydropyridine.<smiles>COC(=O)C1=C(C)NC(C)=C(C(=O)OC)C1(C)C(C)C</smiles>

$3 a$

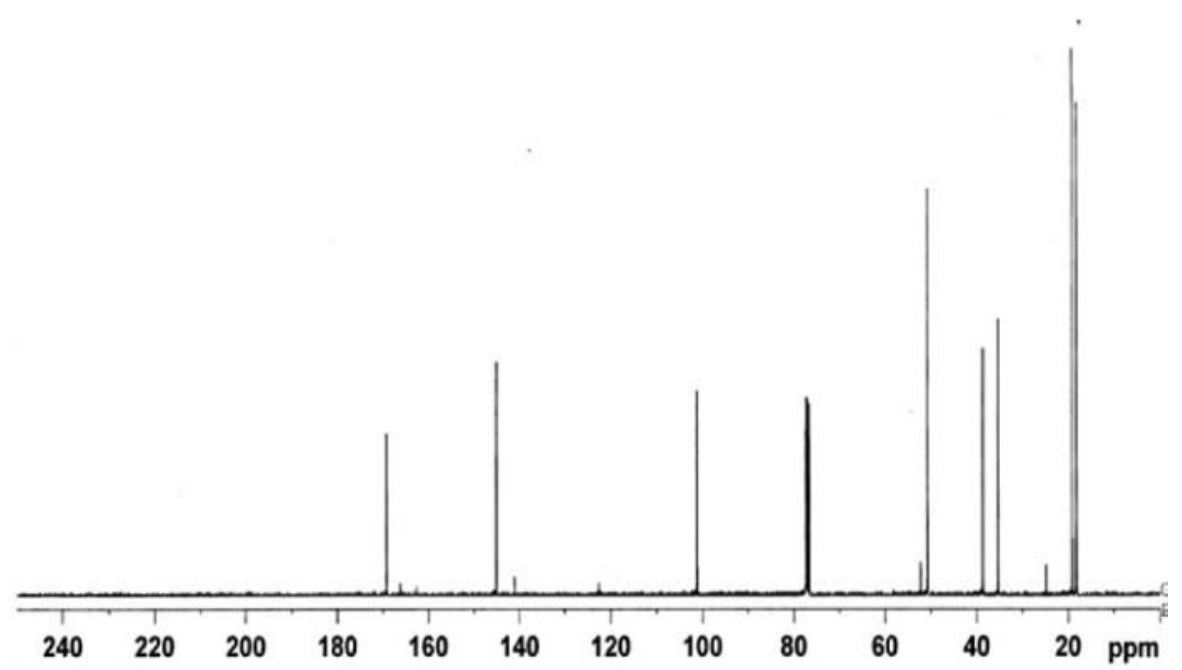

Figure S3. ${ }^{13} \mathrm{C}$ NMR spectrum (100 MHz, $\mathrm{CDCl}_{3}$ ) of 2,6-dimethyl-4-(iso-propyl)-3,5-dicarbomethoxy-1,4-dihydropyridine. 


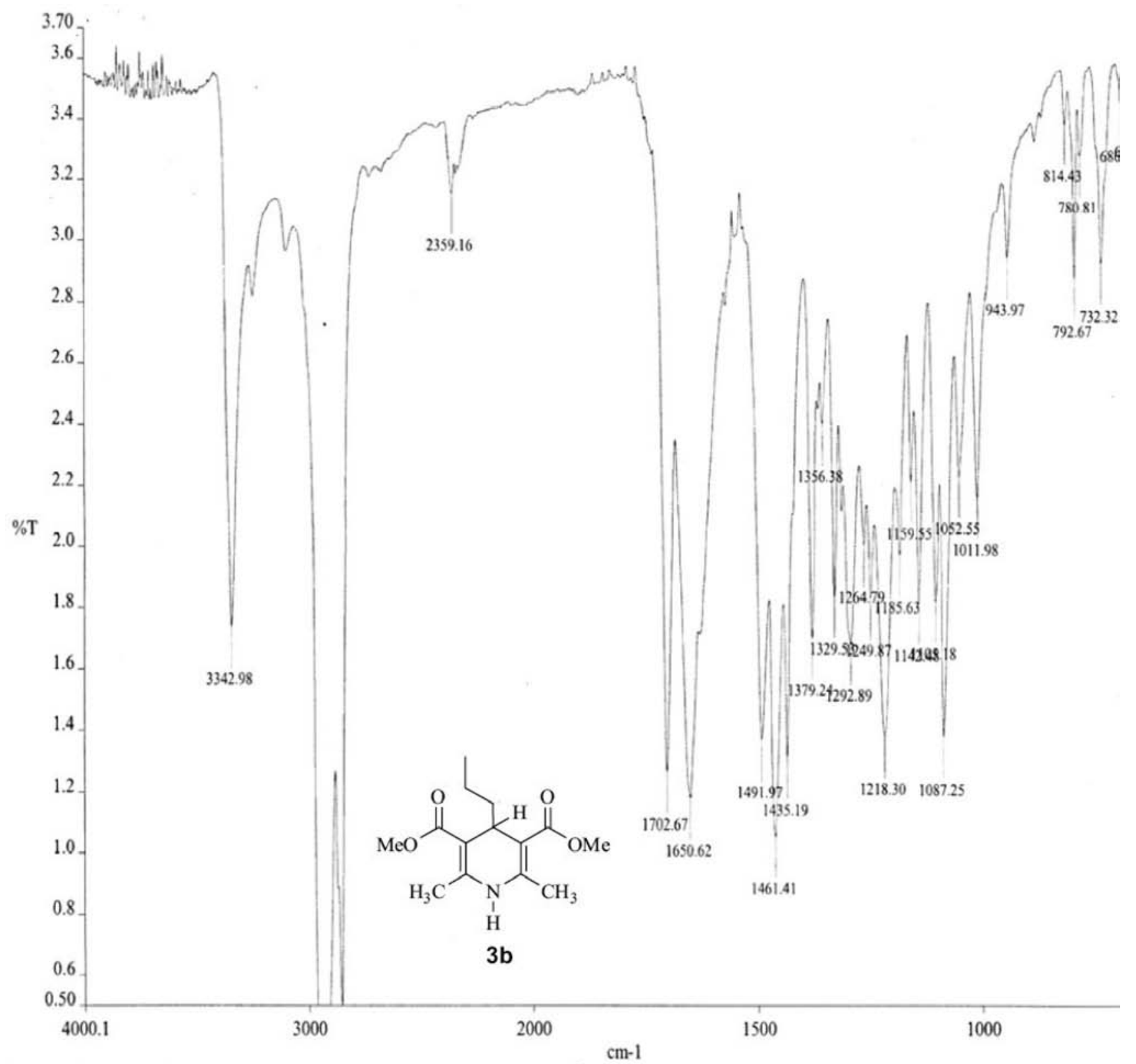

Figure S4. IR spectrum of 2,6-dimethyl-4-(n-propyl)-3,5-dicarbomethoxy-1,4-dihydropyridine. 
<smiles>CCCC1C(C(=O)OC)=C(C)NC(C)=C1C(=O)OC</smiles>

$3 b$

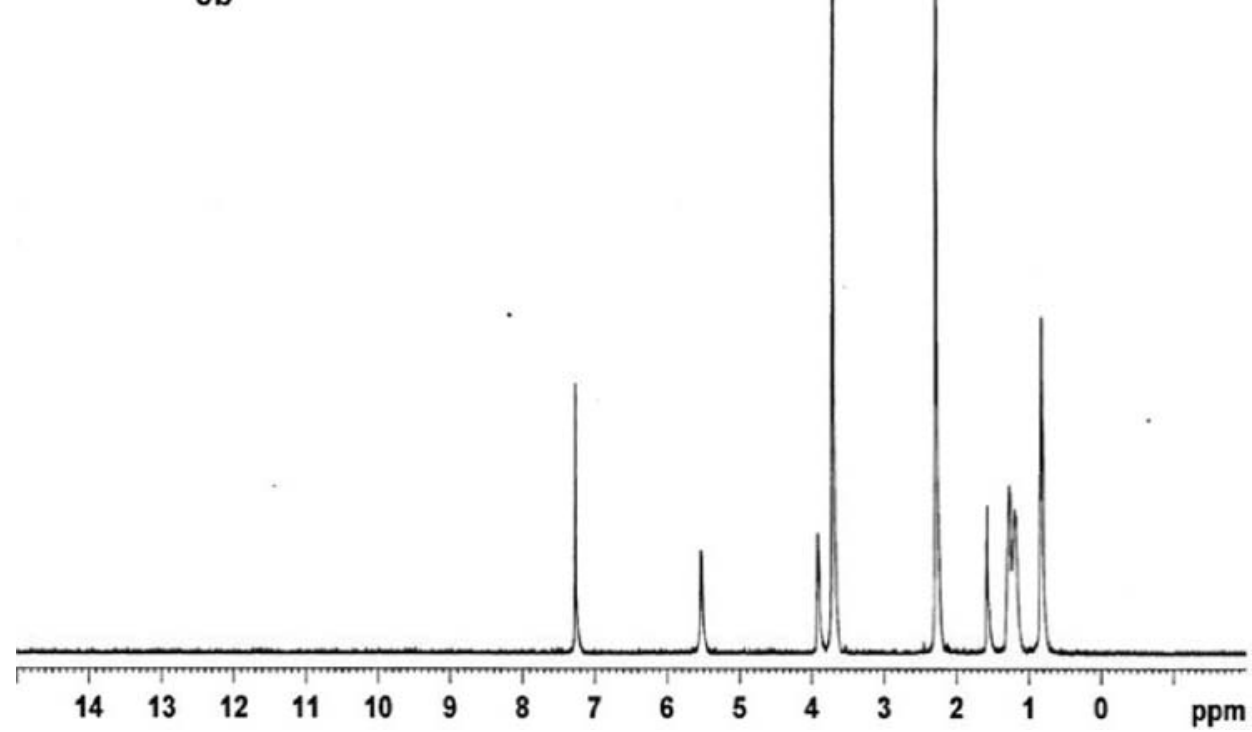

Figure S5. ${ }^{1} \mathrm{H}$ NMR spectrum (400 MHz, $\mathrm{CDCl}_{3}$ ) of 2,6-dimethyl-4-(n-propyl)-3,5-dicarbomethoxy-1,4-dihydropyridine.<smiles>CCCC1C(C(=O)OC)=C(C)NC(C)=C1C(=O)OC</smiles>

$3 \mathbf{b}$

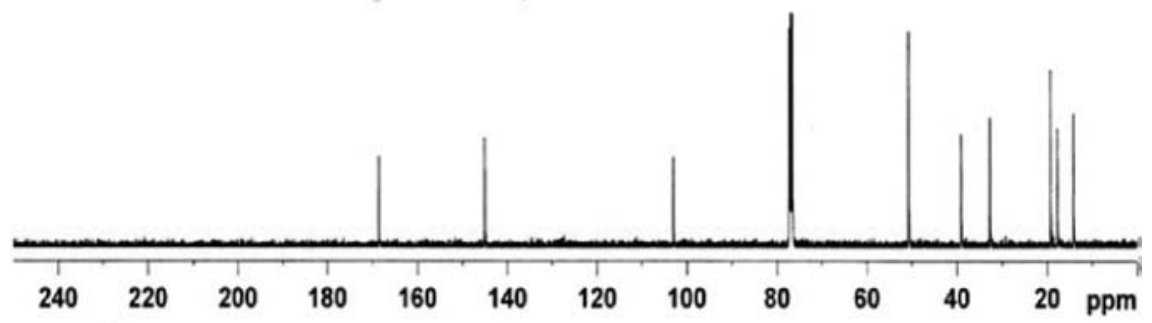

Figure S6. ${ }^{13} \mathrm{C}$ NMR spectrum (100 MHz, $\mathrm{CDCl}_{3}$ ) of 2,6-dimethyl-4-(n-propyl)-3,5-dicarbomethoxy-1,4-dihydropyridine. 


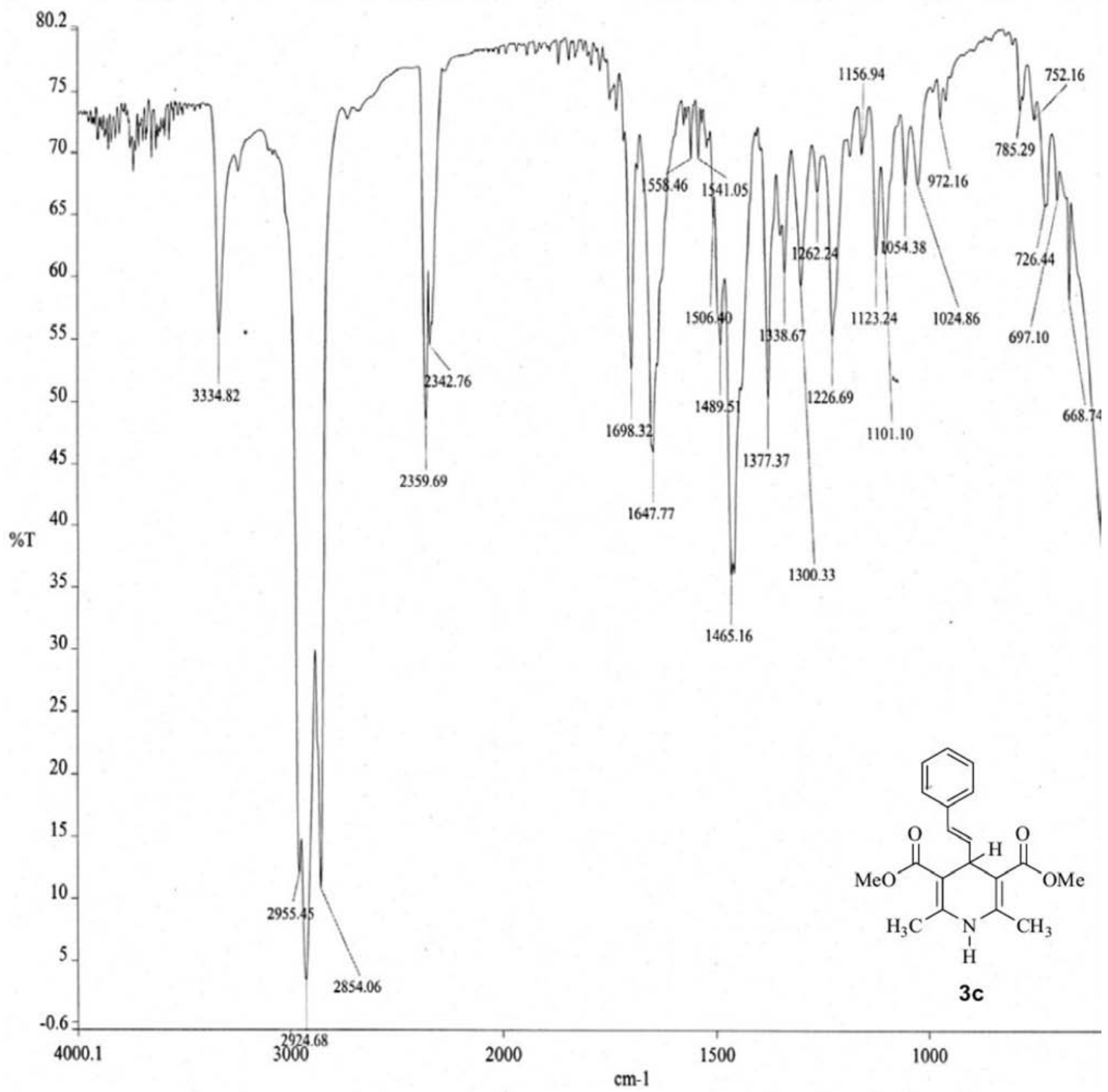

Figure S7. IR spectrum of 2,6-dimethyl-4-(2-phenylethylene)-3,5-dicarbomethoxy-1,4-dihydropyridine. 


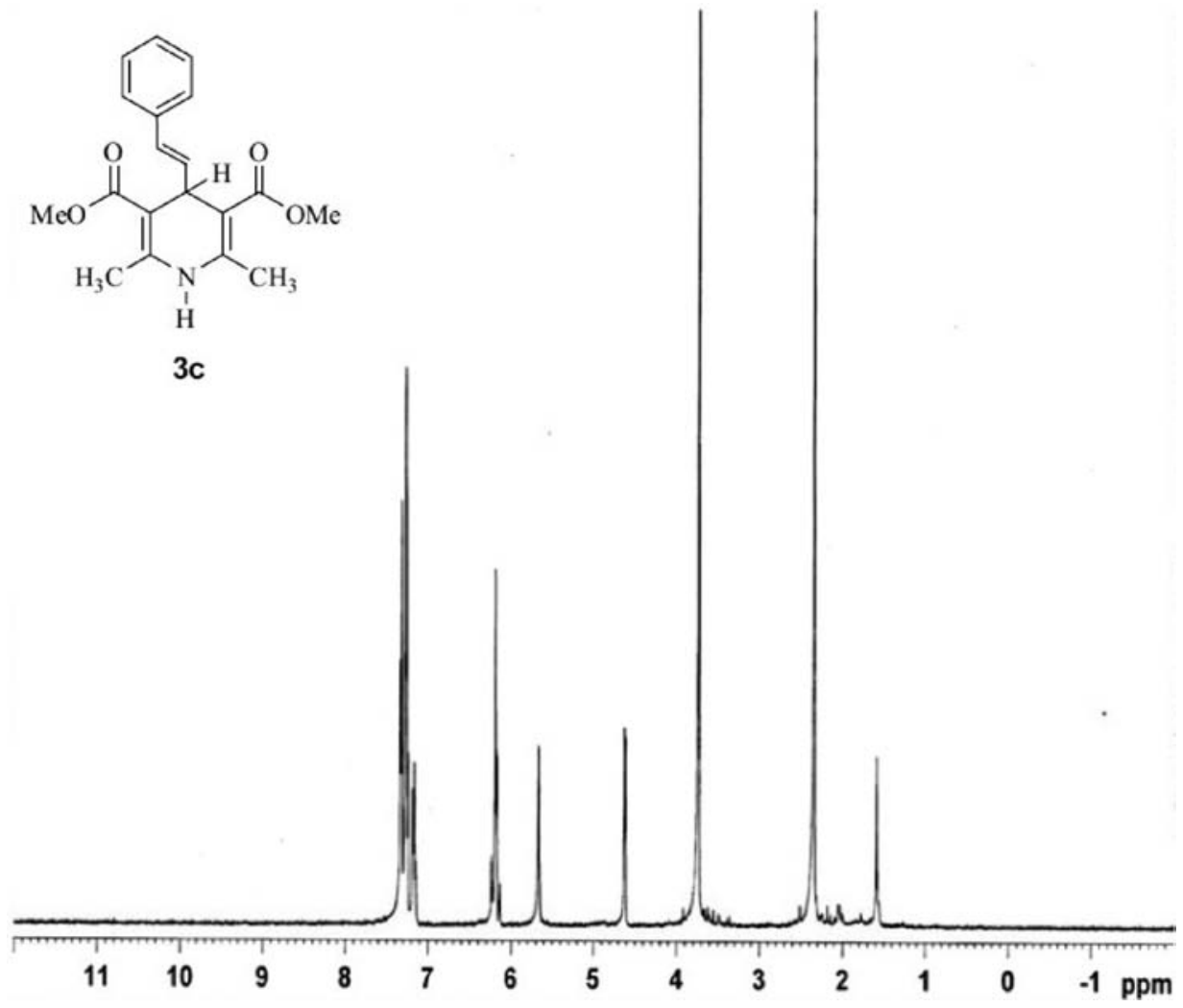

Figure S8. ${ }^{1} \mathrm{H}$ NMR spectrum (400 MHz, $\mathrm{CDCl}_{3}$ ) of 2,6-dimethyl-4-(2-phenylethylene)-3,5-dicarbomethoxy-1,4-dihydropyridine.<smiles>COC(=O)C1=C(C)NC(C)=C(C(C)=O)C1(/C=C/c1ccccc1)C(=O)OC</smiles>

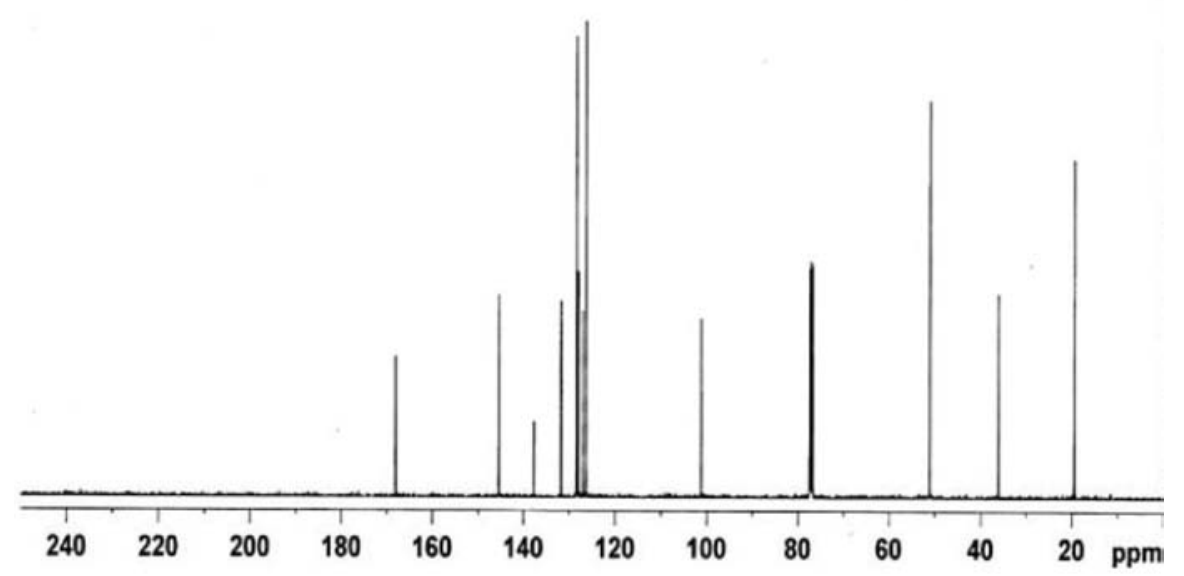

Figure S9. ${ }^{13} \mathrm{C}$ NMR spectrum (100 MHz, $\mathrm{CDCl}_{3}$ ) of 2,6-dimethyl-4-(2-phenylethylene)-3,5-dicarbomethoxy-1,4-dihydropyridine. 


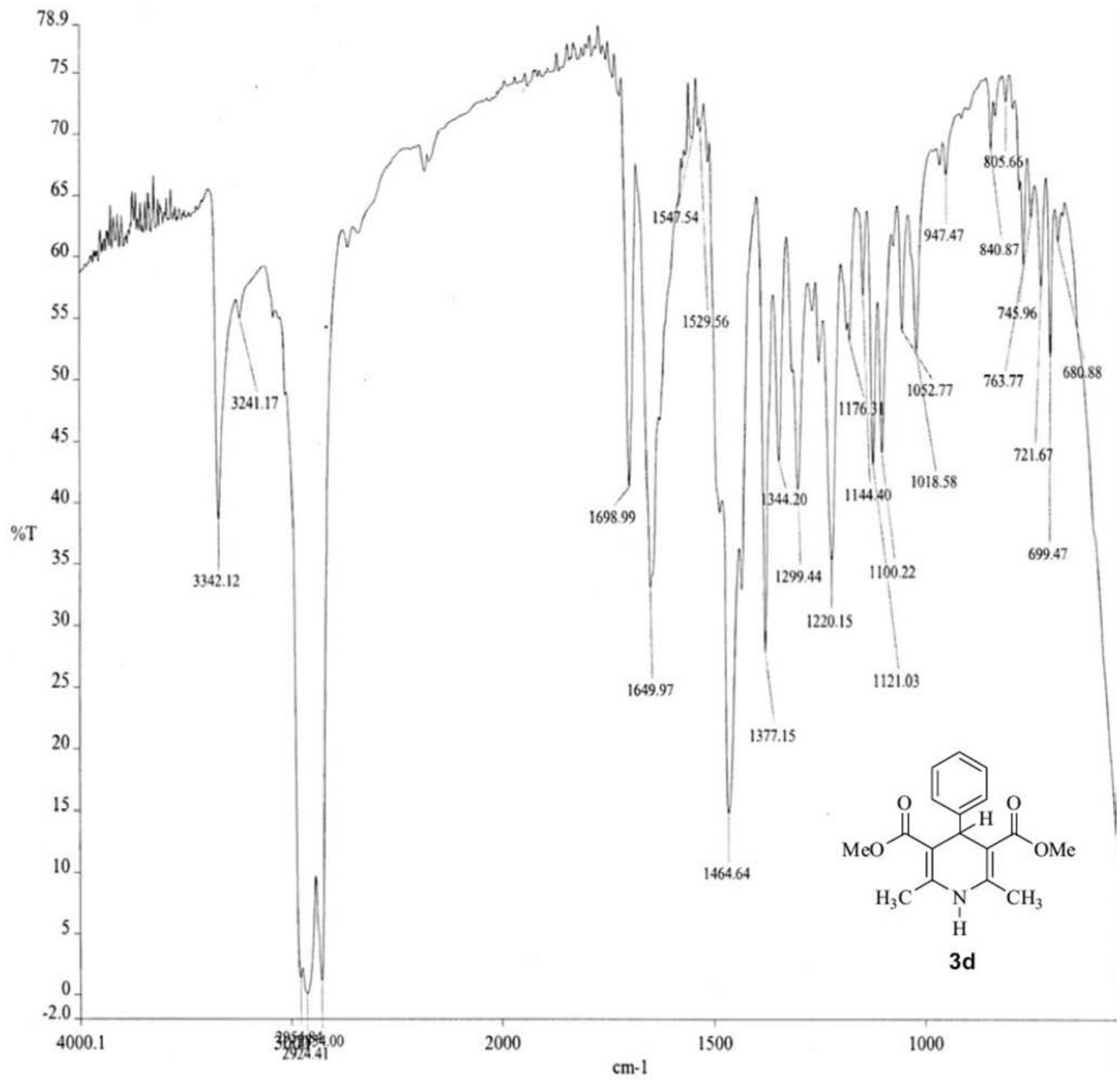

Figure S10. IR spectrum of 2,6-dimethyl-4-(phenyl)-3,5-dicarbomethoxy-1,4-dihydropyridine. 


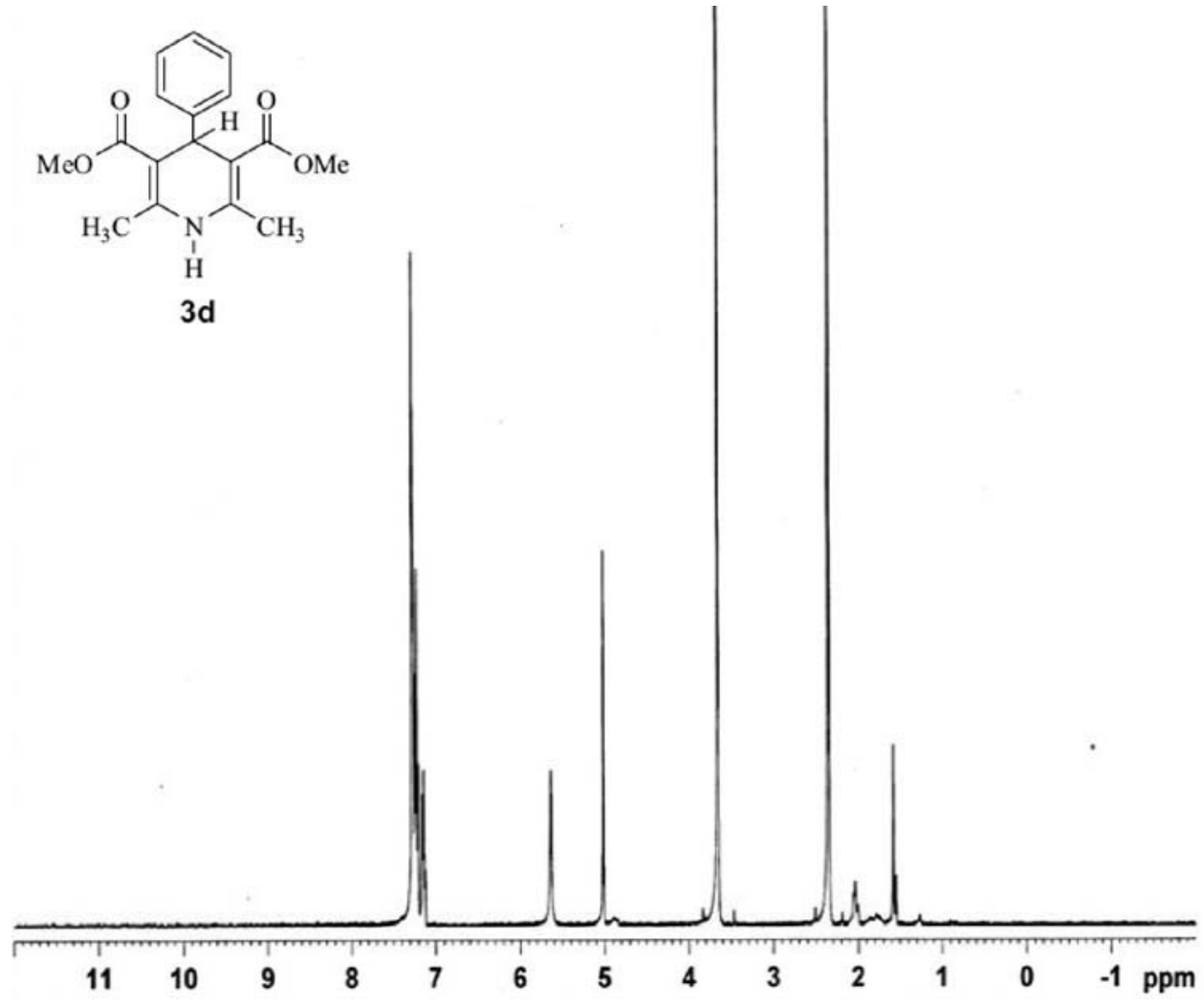

Figure S11. ${ }^{1} \mathrm{H}$ NMR spectrum (400 MHz, $\mathrm{CDCl}_{3}$ ) of 2,6-dimethyl-4-(phenyl)-3,5-dicarbomethoxy-1,4-dihydropyridine.<smiles>COC(=O)C1=C(C)N([13CH3])C(C)=C(C(C)=O)C1c1ccccc1</smiles>

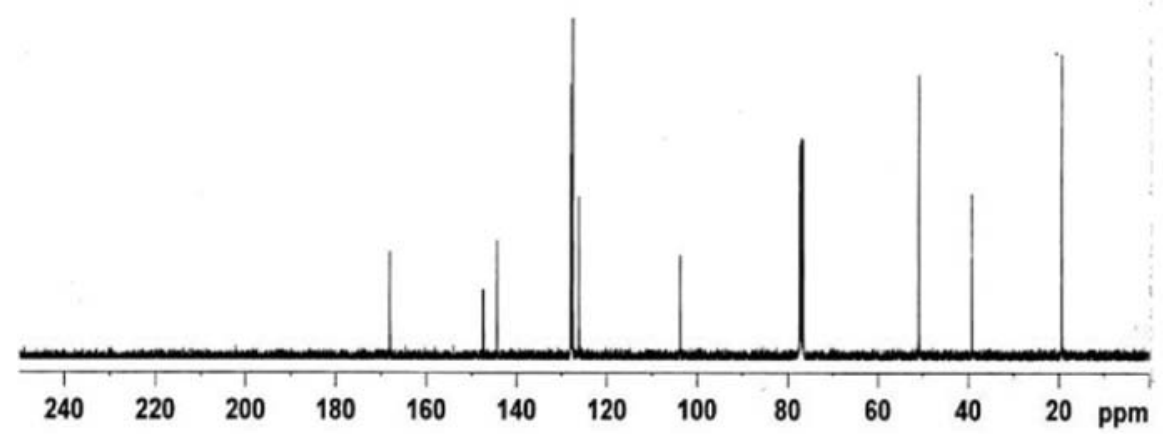

Figure S12. ${ }^{13} \mathrm{C}$ NMR spectrum (100 MHz, $\mathrm{CDCl}_{3}$ ) of 2,6-dimethyl-4-(phenyl)-3,5-dicarbomethoxy-1,4-dihydropyridine. 


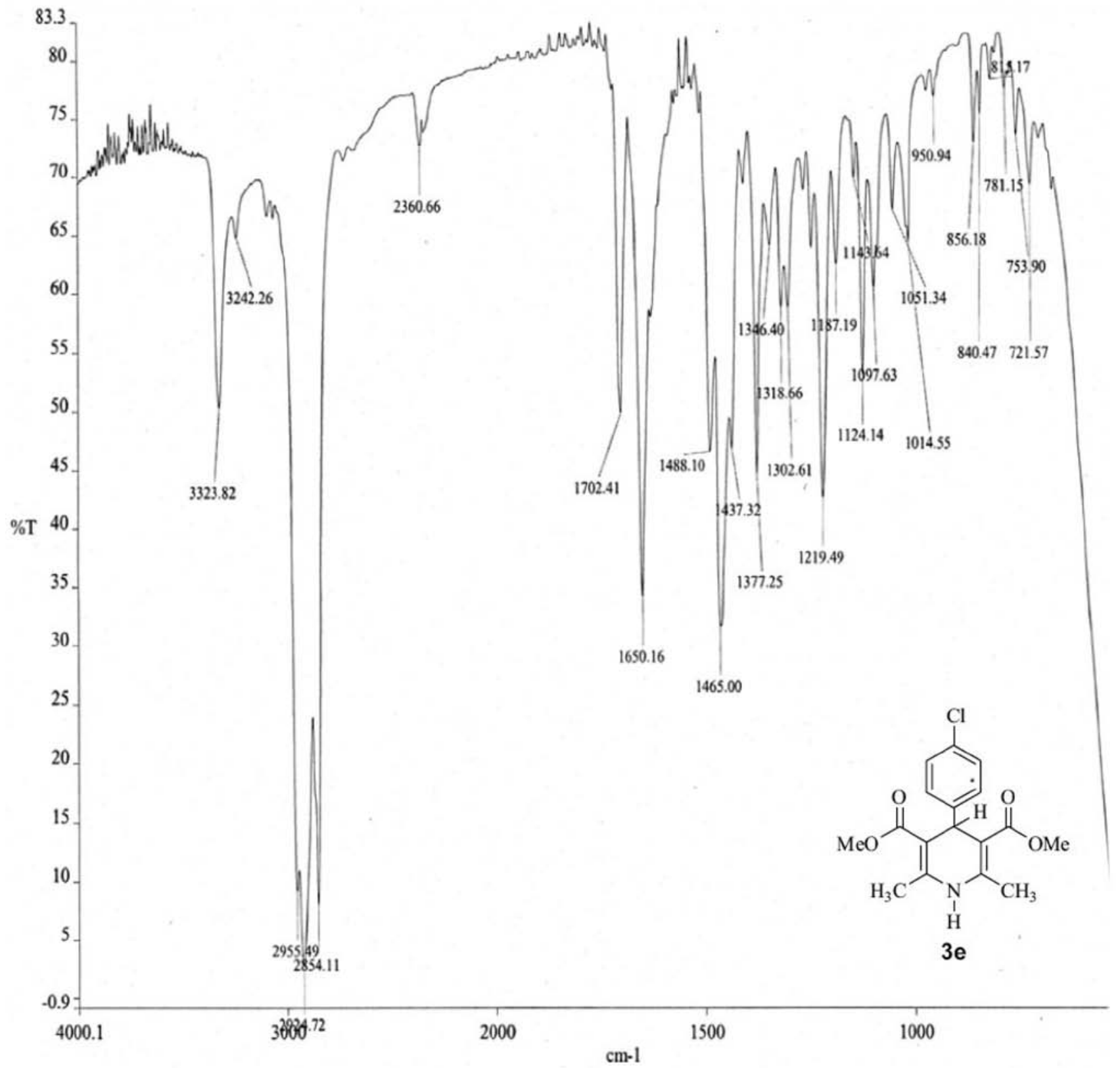

Figure S13. IR spectrum of 2,6-dimethyl-4-(4-chlorophenyl)-3,5-dicarbomethoxy-1,4-dihydropyridine. 
<smiles>COC(=O)C1=C(C)N(C)C(C)=C(C(=O)OC)C1c1ccc(Cl)cc1</smiles>

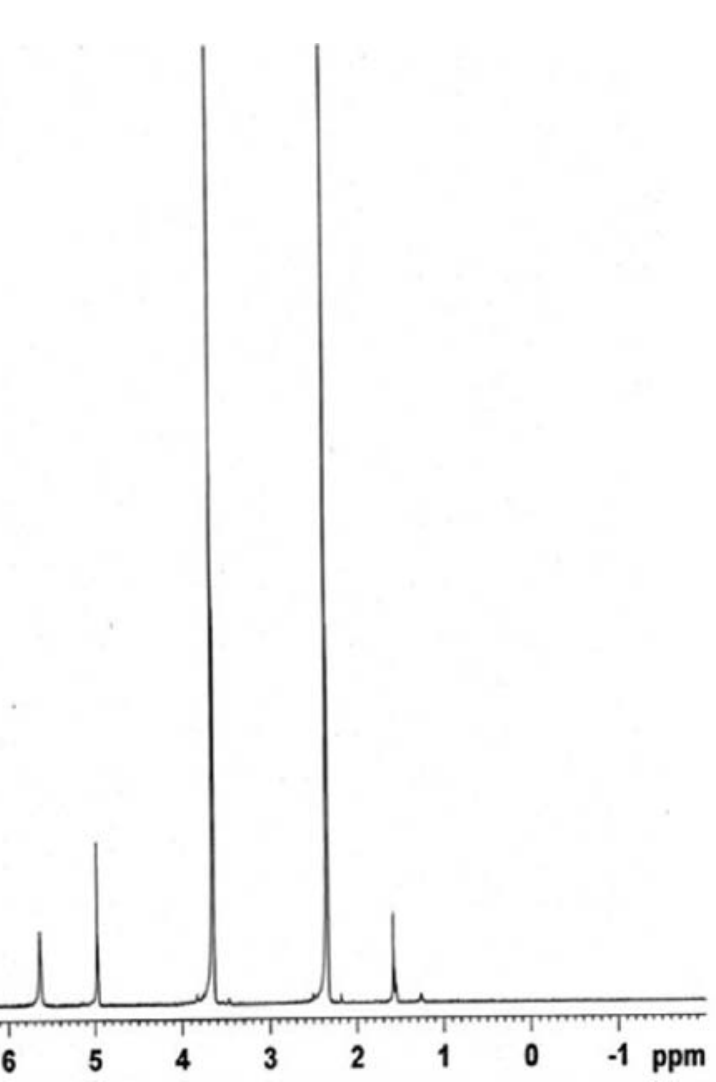

Figure S14. ${ }^{1} \mathrm{H}$ NMR spectrum (400 MHz, $\mathrm{CDCl}_{3}$ ) of 2,6-dimethyl-4-(4-chlorophenyl)-3,5-dicarbomethoxy-1,4-dihydropyridine.<smiles>COC(=O)C1=C(C)N(C)C(C)=C(C(=O)OC)C1c1ccc(Cl)cc1</smiles>

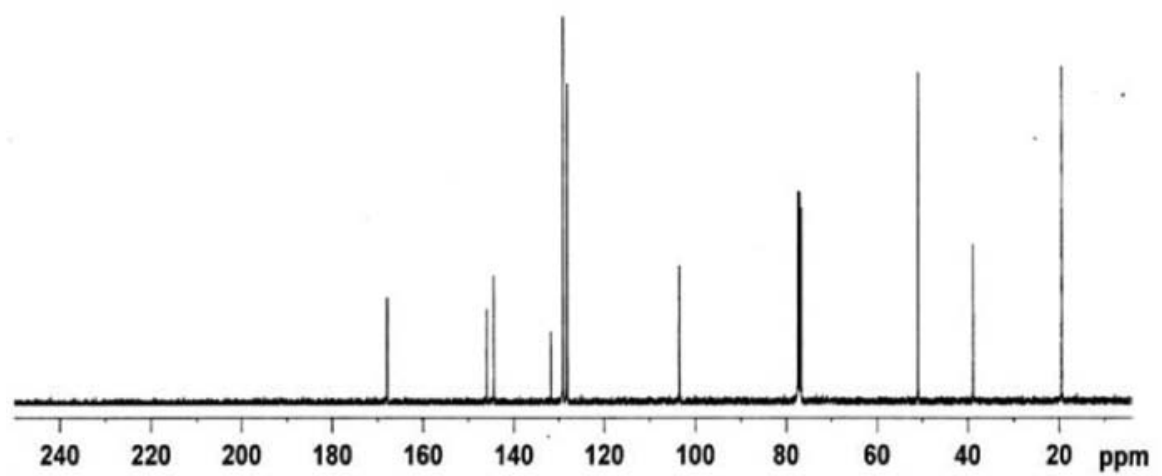

Figure S15. ${ }^{13} \mathrm{C}$ NMR spectrum (100 MHz, $\mathrm{CDCl}_{3}$ ) of 2,6-dimethyl-4-(4-chlorophenyl)-3,5-dicarbomethoxy-1,4-dihydropyridine. 


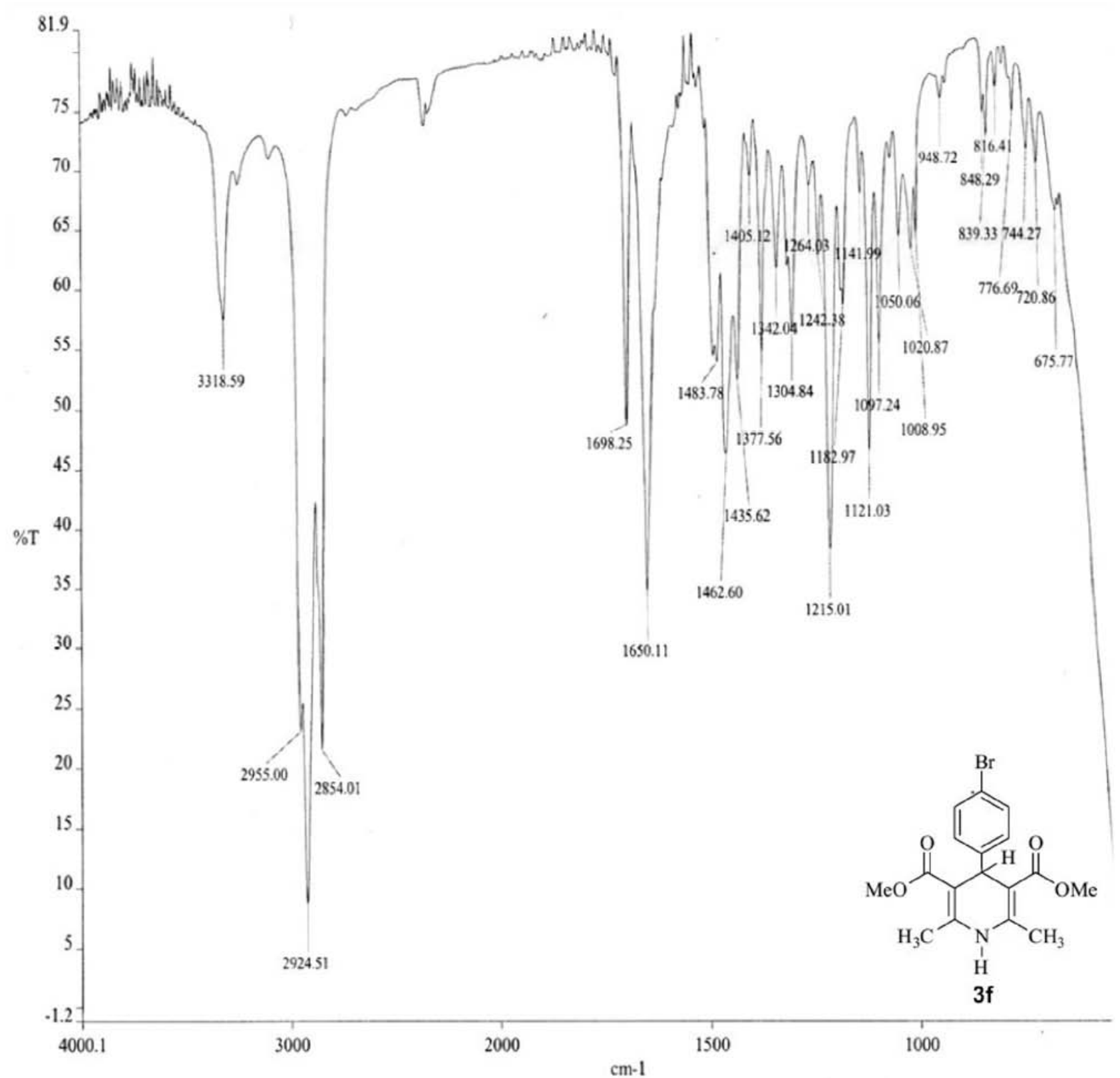

Figure S16. IR spectrum of 2,6-dimethyl-4-(4-bromophenyl)-3,5-dicarbomethoxy-1,4-dihydropyridine. 
<smiles>COC(=O)C1=C(C)N([AlH2])C(C)=C(C(=O)OC)C1(c1ccccc1)c1ccc(Br)cc1</smiles>

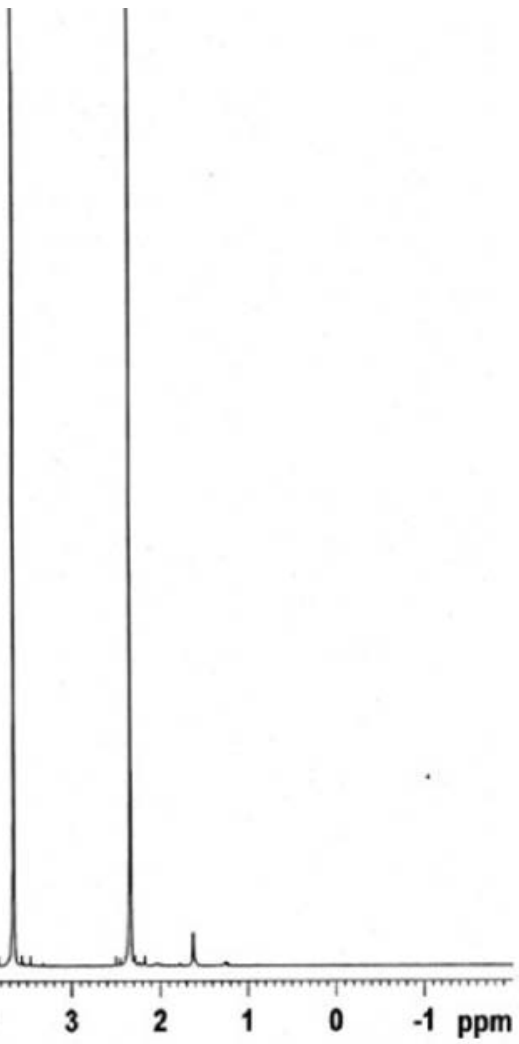

Figure S17. ${ }^{1} \mathrm{H}$ NMR spectrum (400 $\mathrm{MHz}, \mathrm{CDCl}_{3}$ ) of 2,6-dimethyl-4-(4-bromophenyl)-3,5-dicarbomethoxy-1,4-dihydropyridine.<smiles>COC(=O)C1=C(C)N([AlH2])C(C)=C(C(=O)OC)C1c1ccc(Br)cc1</smiles>

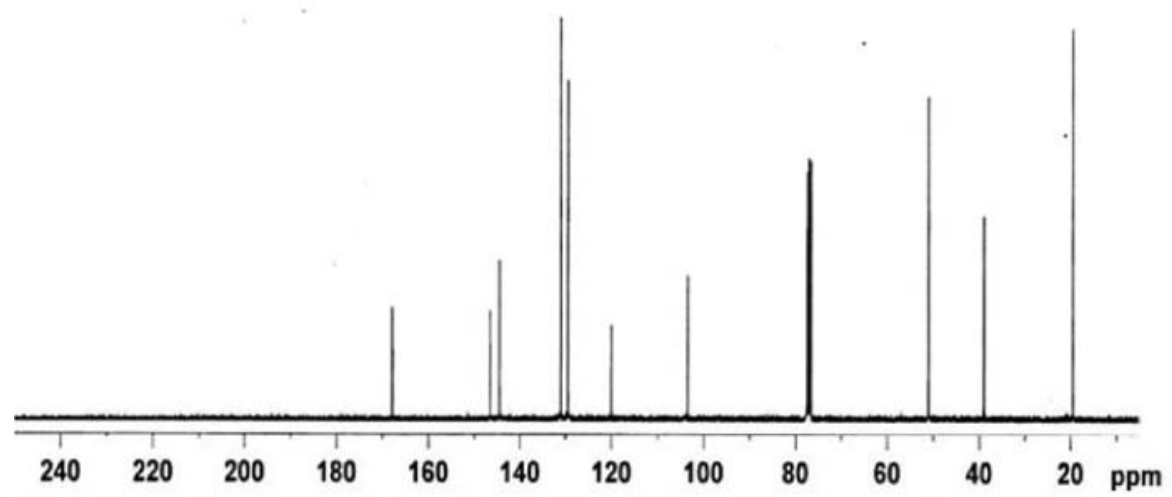

Figure S18. ${ }^{13} \mathrm{C}$ NMR spectrum (100 MHz, $\mathrm{CDCl}_{3}$ ) of 2,6-dimethyl-4-(4-bromophenyl)-3,5-dicarbomethoxy-1,4-dihydropyridine. 


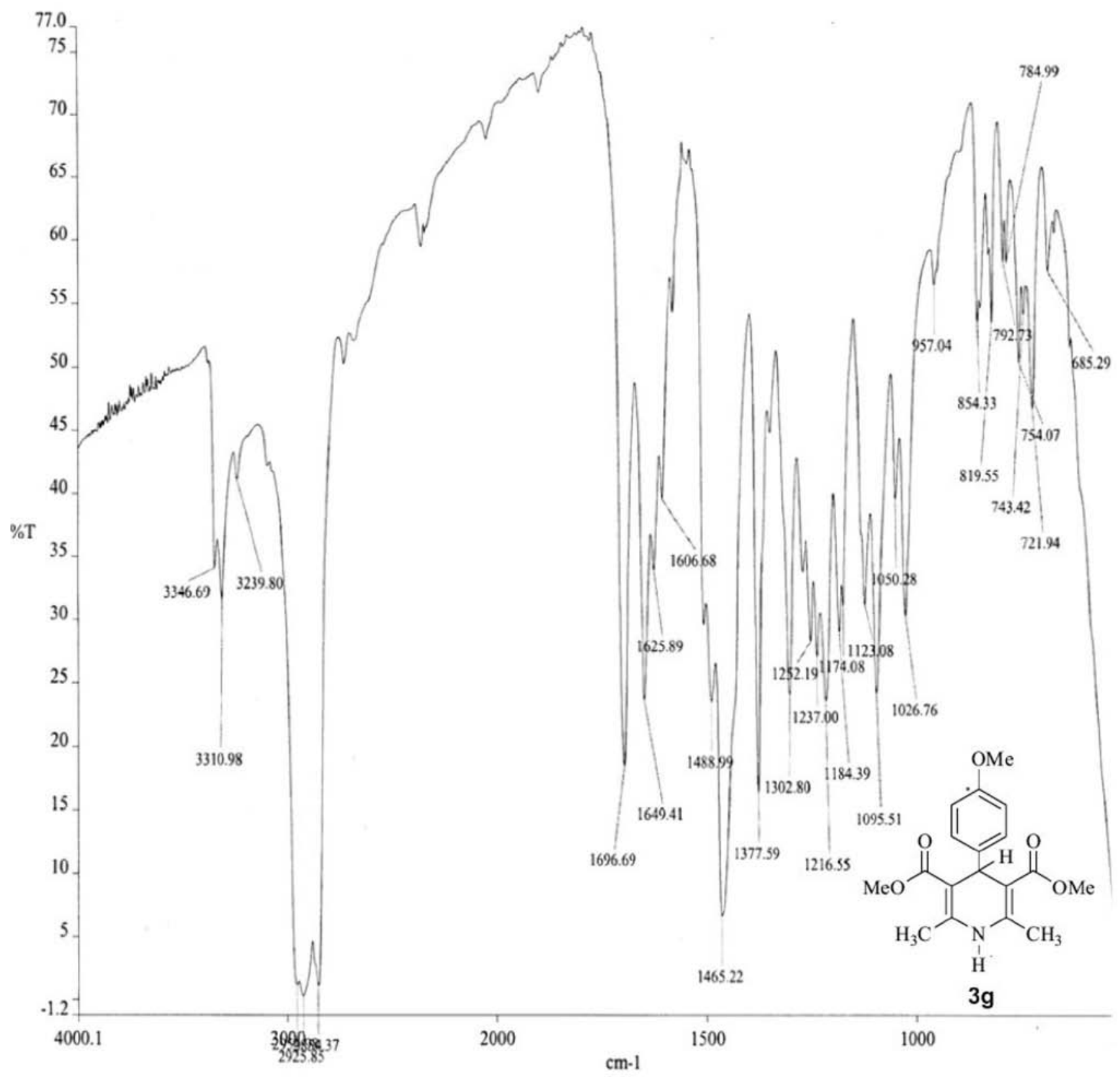

Figure S19. IR spectrum of 2,6-dimethyl-4-(4-methoxyphenyl)-3,5-dicarbomethoxy-1,4-dihydropyridine. 
<smiles>COC(=O)C1=C(C)N([13CH3])C(C)=C(C(=O)OC)C1(c1ccccc1)c1ccc(OC)cc1</smiles>

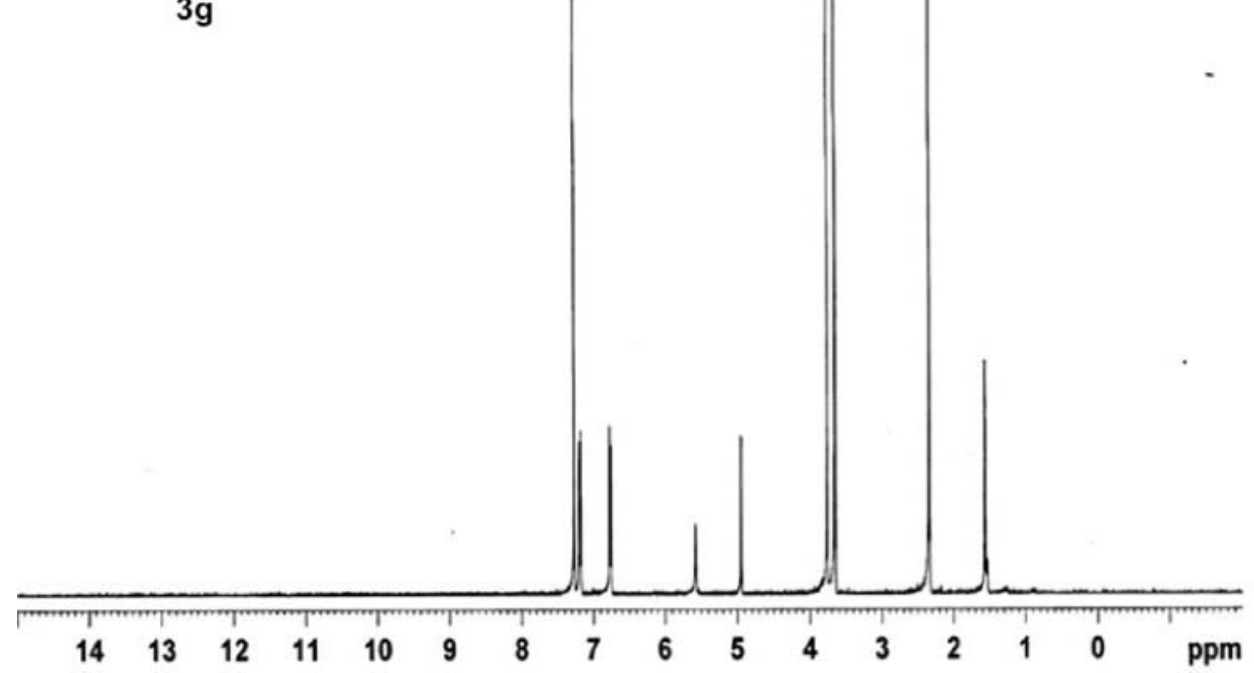

Figure S20. ${ }^{1} \mathrm{H}$ NMR spectrum (400 MHz, $\mathrm{CDCl}_{3}$ ) of 2,6-dimethyl-4-(4-methoxyphenyl)-3,5-dicarbomethoxy-1,4-dihydropyridine.<smiles>CCCCCCN1C(C)=C(C(C)=O)C(c2ccc(OC)cc2)C(C(=O)OC)=C1C</smiles>

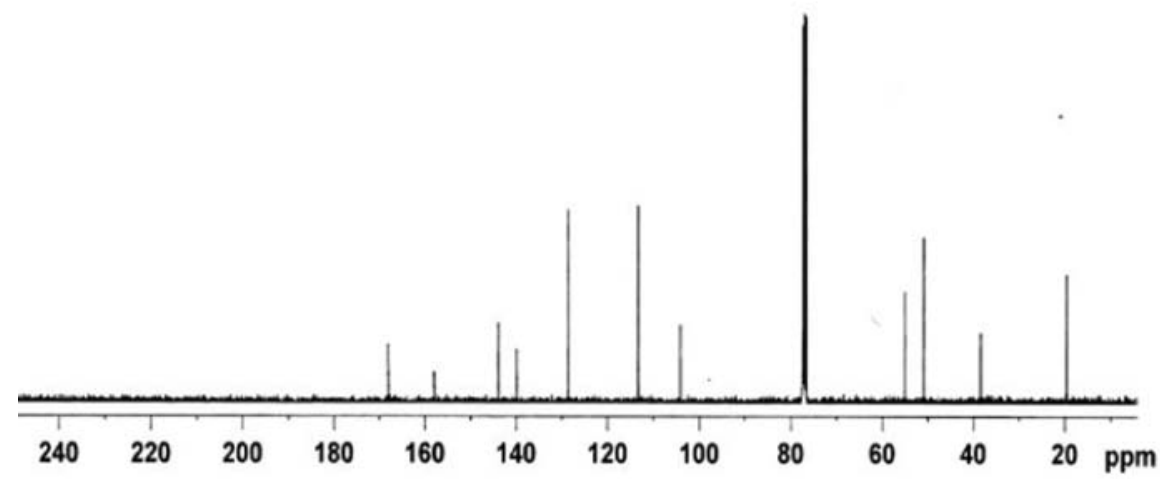

Figure S21. ${ }^{13} \mathrm{C}$ NMR spectrum (100 MHz, $\mathrm{CDCl}_{3}$ ) of 2,6-dimethyl-4-(4-methoxyphenyl)-3,5-dicarbomethoxy-1,4-dihydropyridine. 


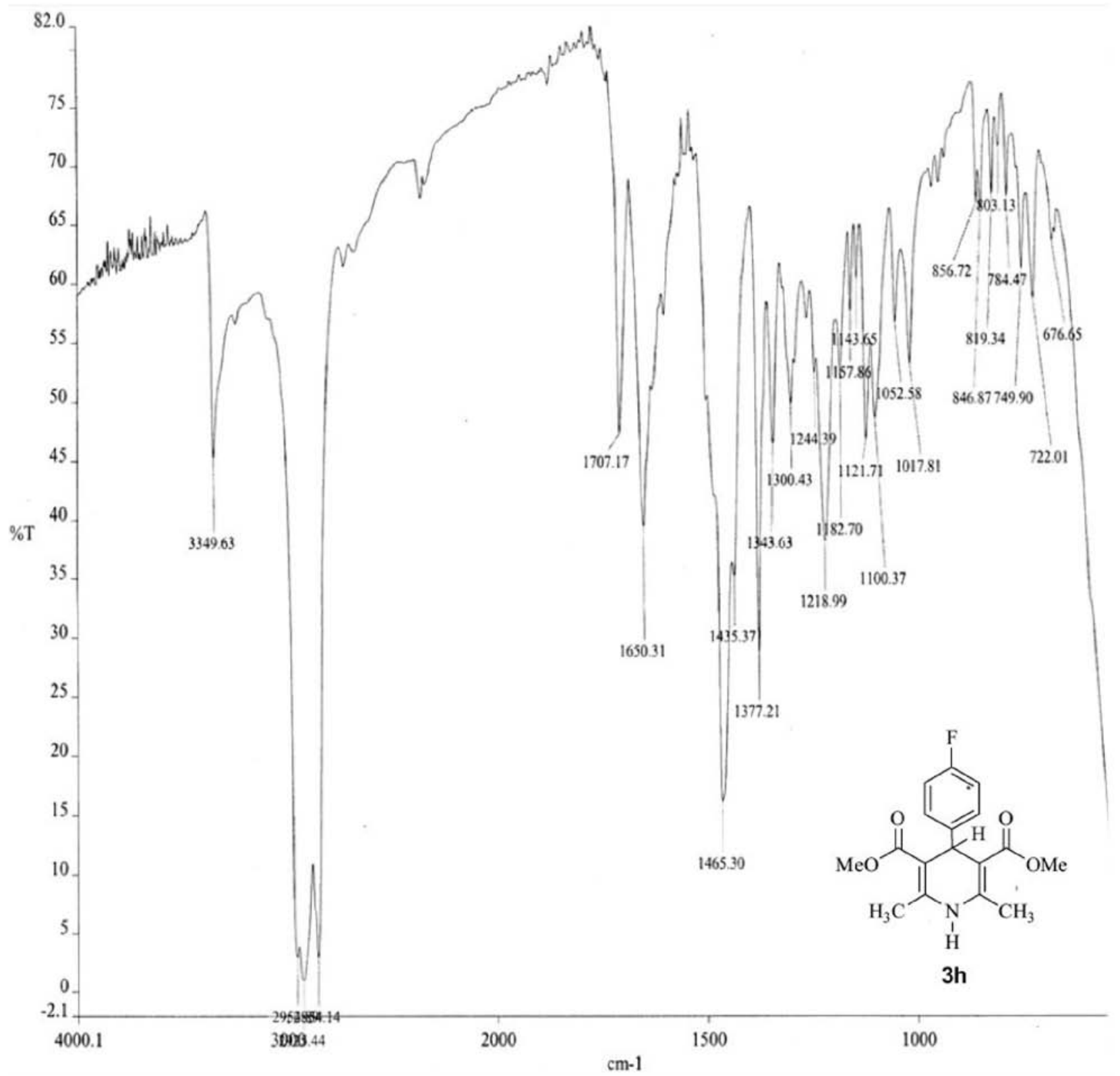

Figure S22. IR spectrum of 2,6-dimethyl-4-(4-flourophenyl)-3,5-dicarbomethoxy-1,4-dihydropyridine. 
<smiles>COC(=O)C1=C(C)N([13CH2]c2ccccc2)C(C(=O)OC)=C(C(=O)OC)C1c1ccc(F)cc1</smiles>

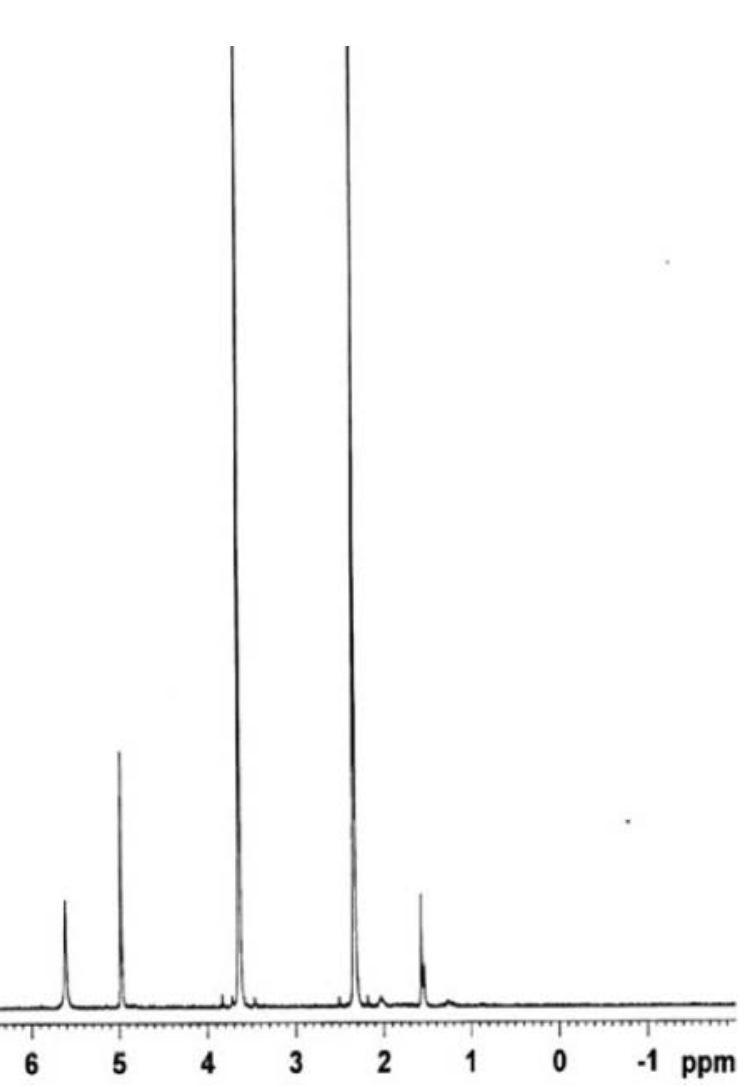

Figure S23. ${ }^{~} \mathrm{H}$ NMR spectrum (400 MHz, $\mathrm{CDCl}_{3}$ ) of 2,6-dimethyl-4-(4-flourophenyl)-3,5-dicarbomethoxy-1,4-dihydropyridine.<smiles>COC(=O)C1=C(C)N([CH+]c2ccccc2)C(C)=C(C(=O)OC)C1c1ccc(F)cc1</smiles>

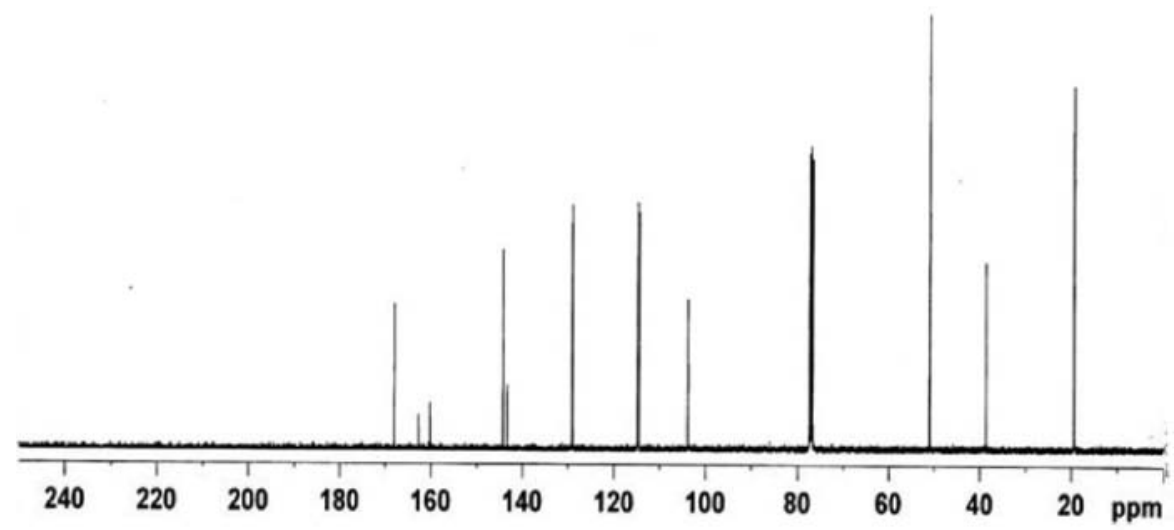

Figure S24. ${ }^{13} \mathrm{C}$ NMR spectrum (100 MHz, $\mathrm{CDCl}_{3}$ ) of 2,6-dimethyl-4-(4-flourophenyl)-3,5-dicarbomethoxy-1,4-dihydropyridine. 


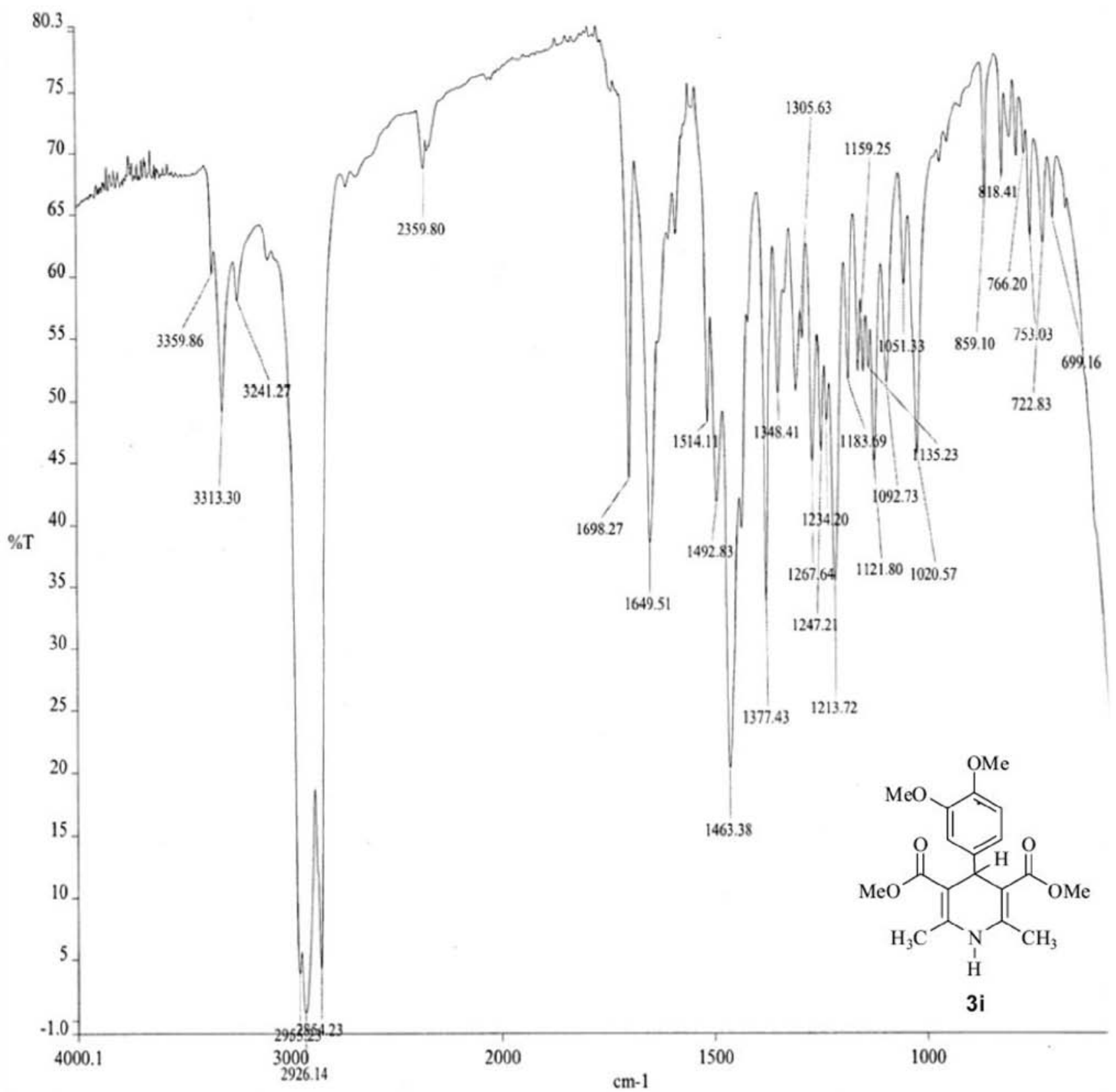

Figure S25. IR spectrum of 2,6-dimethyl-4-(3,4-dimethoxyphenyl)-3,5-dicarbomethoxy-1,4-dihydropyridine. 
<smiles>COC(=O)C1=C(C)NC([AlH2])=C(C(=O)OC)C1c1ccc(OC)c(OC)c1</smiles>

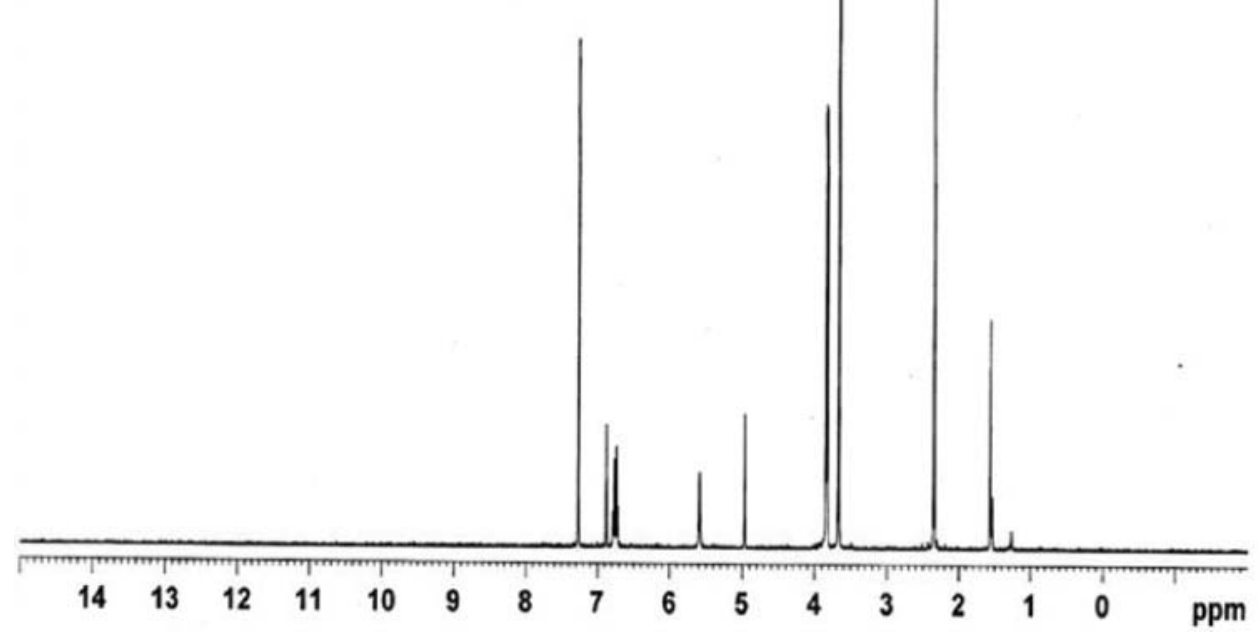

Figure S26. ${ }^{1} \mathrm{H}$ NMR spectrum (400 MHz, $\mathrm{CDCl}_{3}$ ) of 2,6-dimethyl-4-(3,4-dimethoxyphenyl)-3,5-dicarbomethoxy-1,4-dihydropyridine.<smiles>COC(=O)C1=C(C)NC(C)=C(C(=O)OC)C1c1ccc(OC)c(OC)c1</smiles>

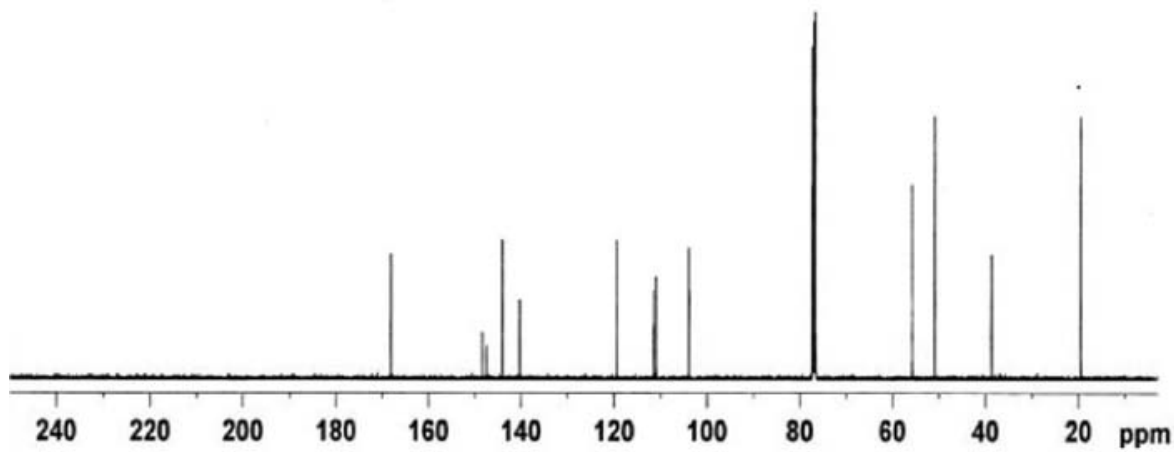

Figure S27. ${ }^{13} \mathrm{C}$ NMR spectrum (100 MHz, $\mathrm{CDCl}_{3}$ ) of 2,6-dimethyl-4-(3,4-dimethoxyphenyl)-3,5-dicarbomethoxy-1,4-dihydropyridine. 


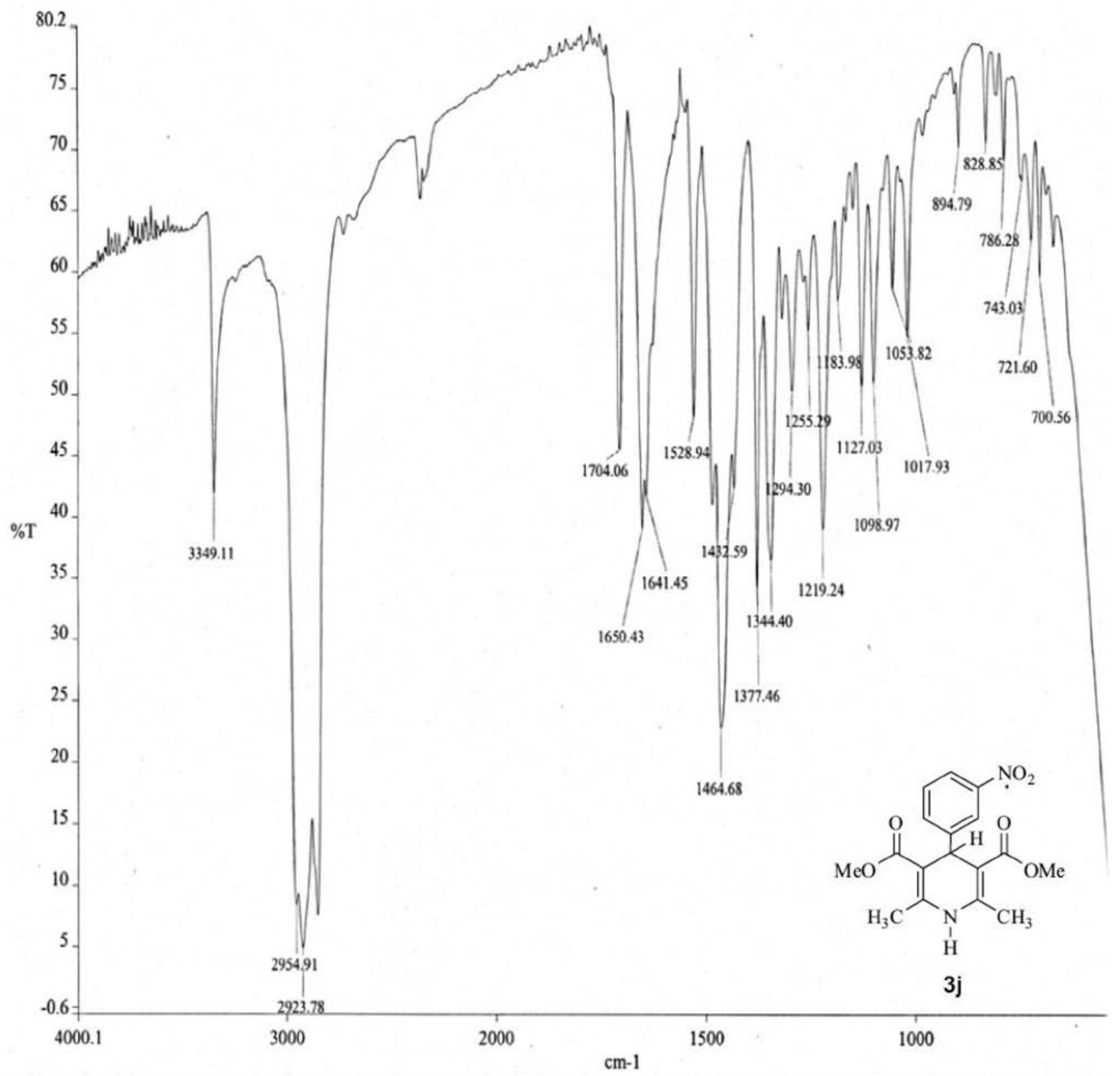

Figure S28. IR spectrum of 2,6-dimethyl-4-(3-nitrophenyl)-3,5-dicarbomethoxy-1,4- dihydropyridine. 
<smiles>COC(=O)C1=C(C)NC(C)=C(C(=O)OC)C1c1cccc([N+](=O)[O-])c1</smiles>

3j

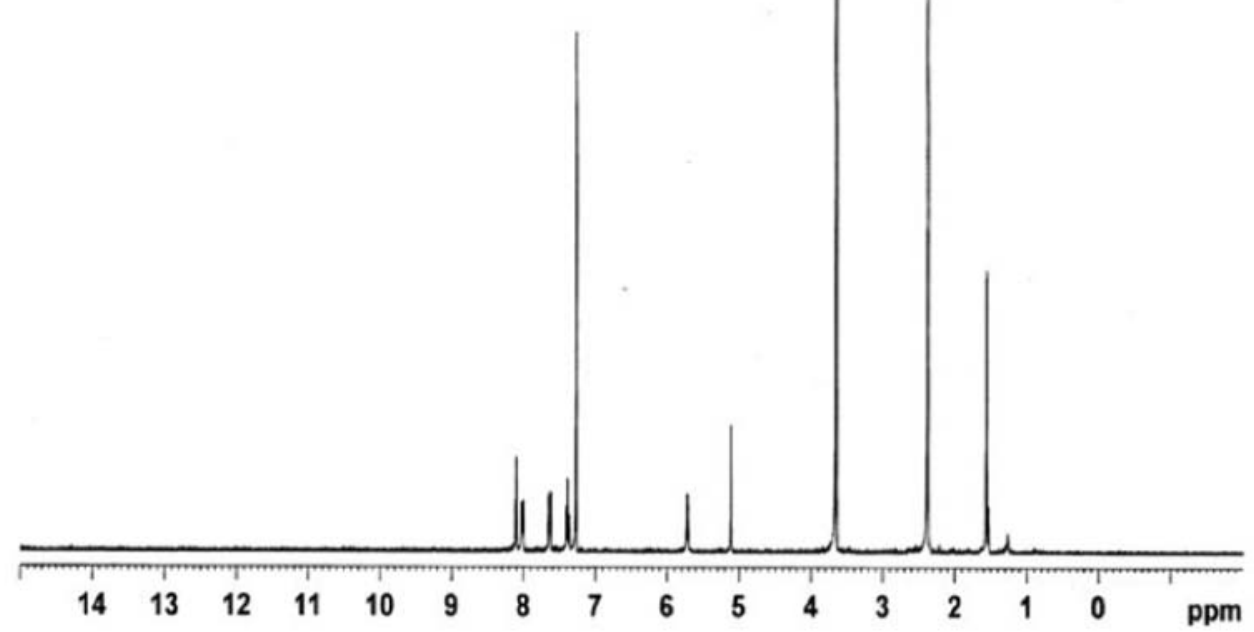

Figure S29. ${ }^{1} \mathrm{H}$ NMR spectrum (400 MHz, $\mathrm{CDCl}_{3}$ ) of 2,6-dimethyl-4-(3-nitrophenyl)-3,5-dicarbomethoxy-1,4- dihydropyridine.<smiles>COC(=O)C1=C(C)NC(C)=C(C(=O)OC)C1(c1ccccc1)c1cccc([N+](=O)[O-])c1</smiles>

3j

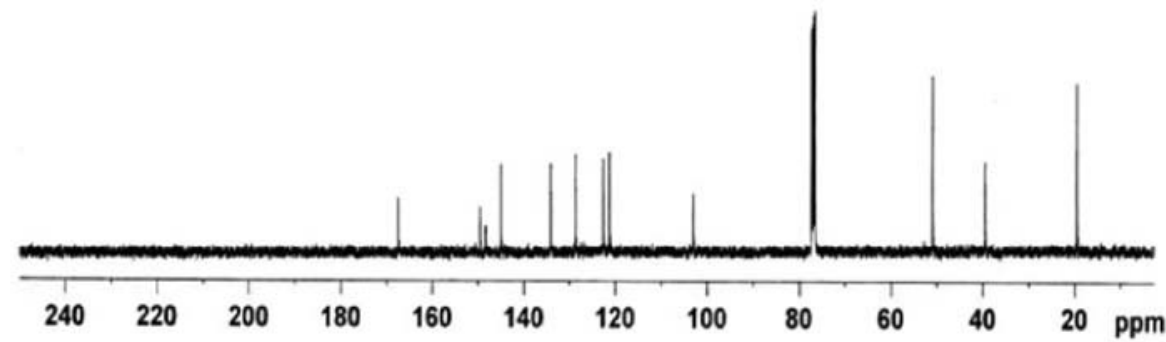

Figure S30. ${ }^{13} \mathrm{C}$ NMR spectrum (100 MHz, $\mathrm{CDCl}_{3}$ ) of 2,6-dimethyl-4-(3-nitrophenyl)-3,5-dicarbomethoxy-1,4- dihydropyridine. 


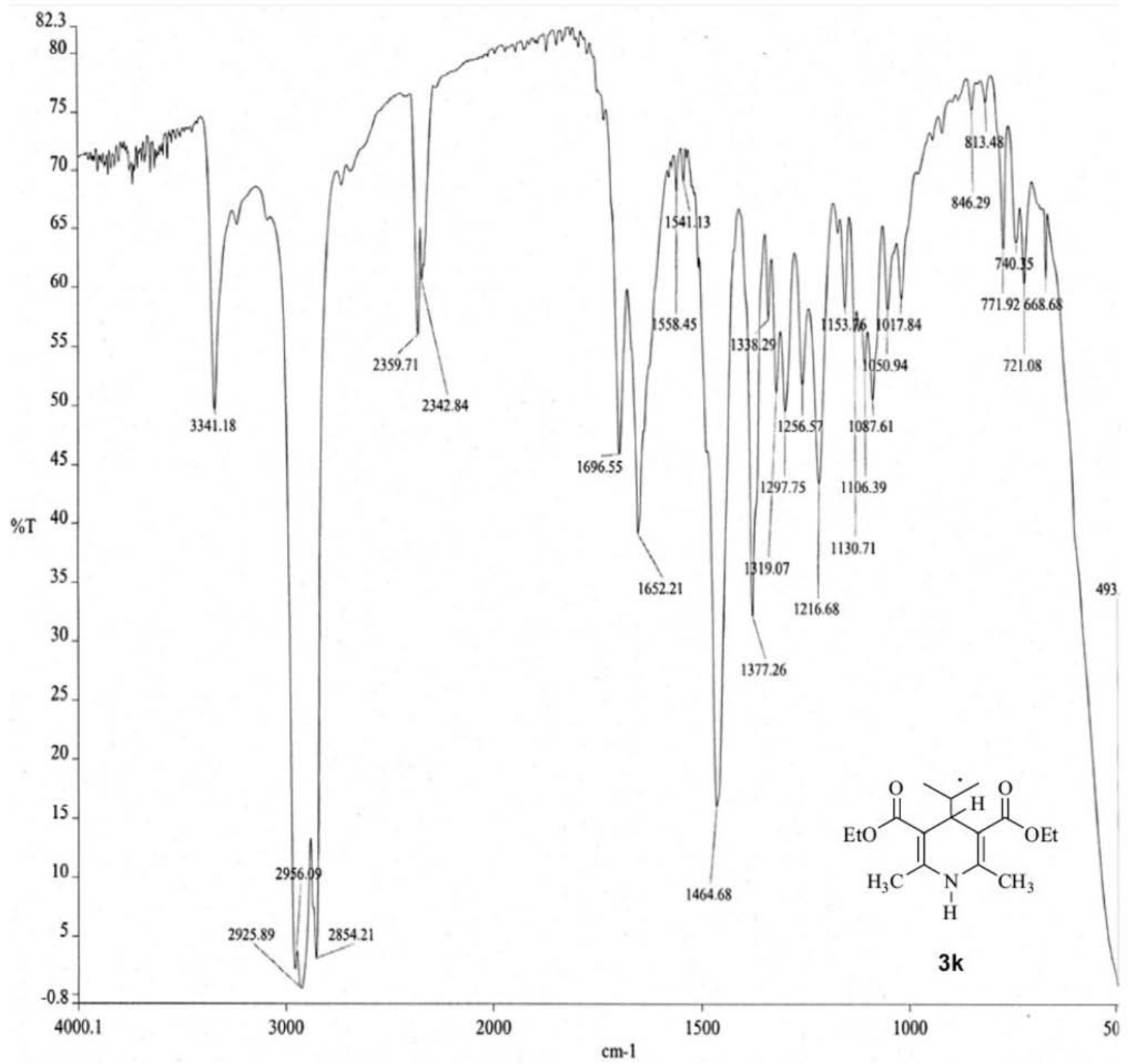

Figure S31. IR spectrum of 2,6-dimethyl-4-(iso-propyl)-3,5-dicarboethoxy-1,4-dihydropyridine. 
<smiles>CCOC(=O)C1=C(C)NC(C)=C(C(=O)OCC)C1(C)C(C)C</smiles>

$3 k$

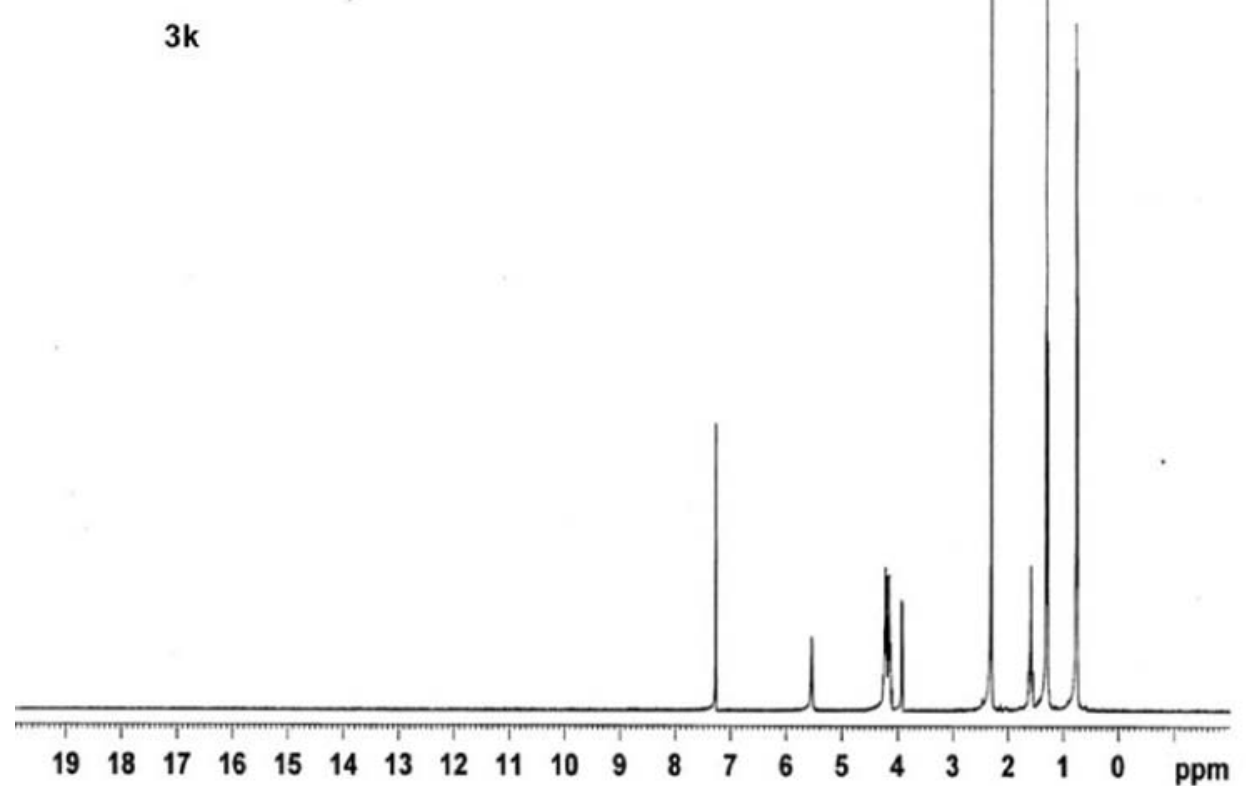

Figure S32. ${ }^{1} \mathrm{H}$ NMR spectrum (400 MHz, $\mathrm{CDCl}_{3}$ ) of 2,6-dimethyl-4-(iso-propyl)-3,5-dicarboethoxy-1,4-dihydropyridine.<smiles>CCOC(=O)C1=C(C)NC(C)=C(C(=O)OCC)C1(C)C(C)C</smiles>

3k

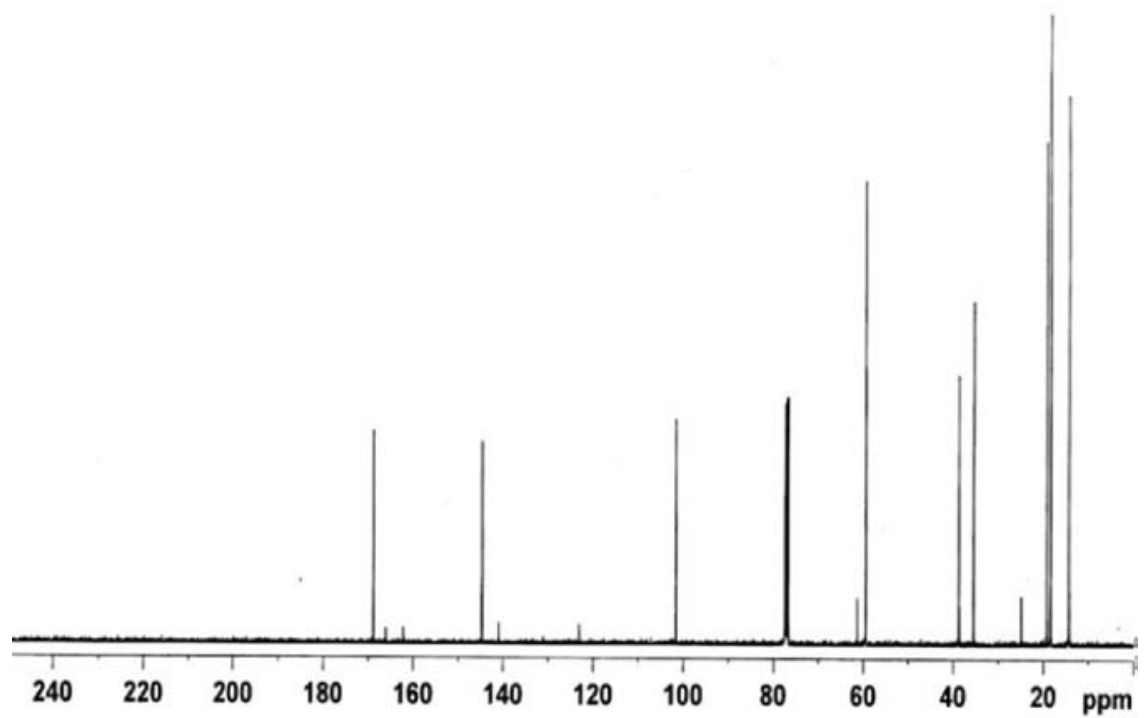

Figure S33. ${ }^{13} \mathrm{C}$ NMR spectrum (100 $\mathrm{MHz}, \mathrm{CDCl}_{3}$ ) of 2,6-dimethyl-4-(iso-propyl)-3,5-dicarboethoxy-1,4-dihydropyridine. 


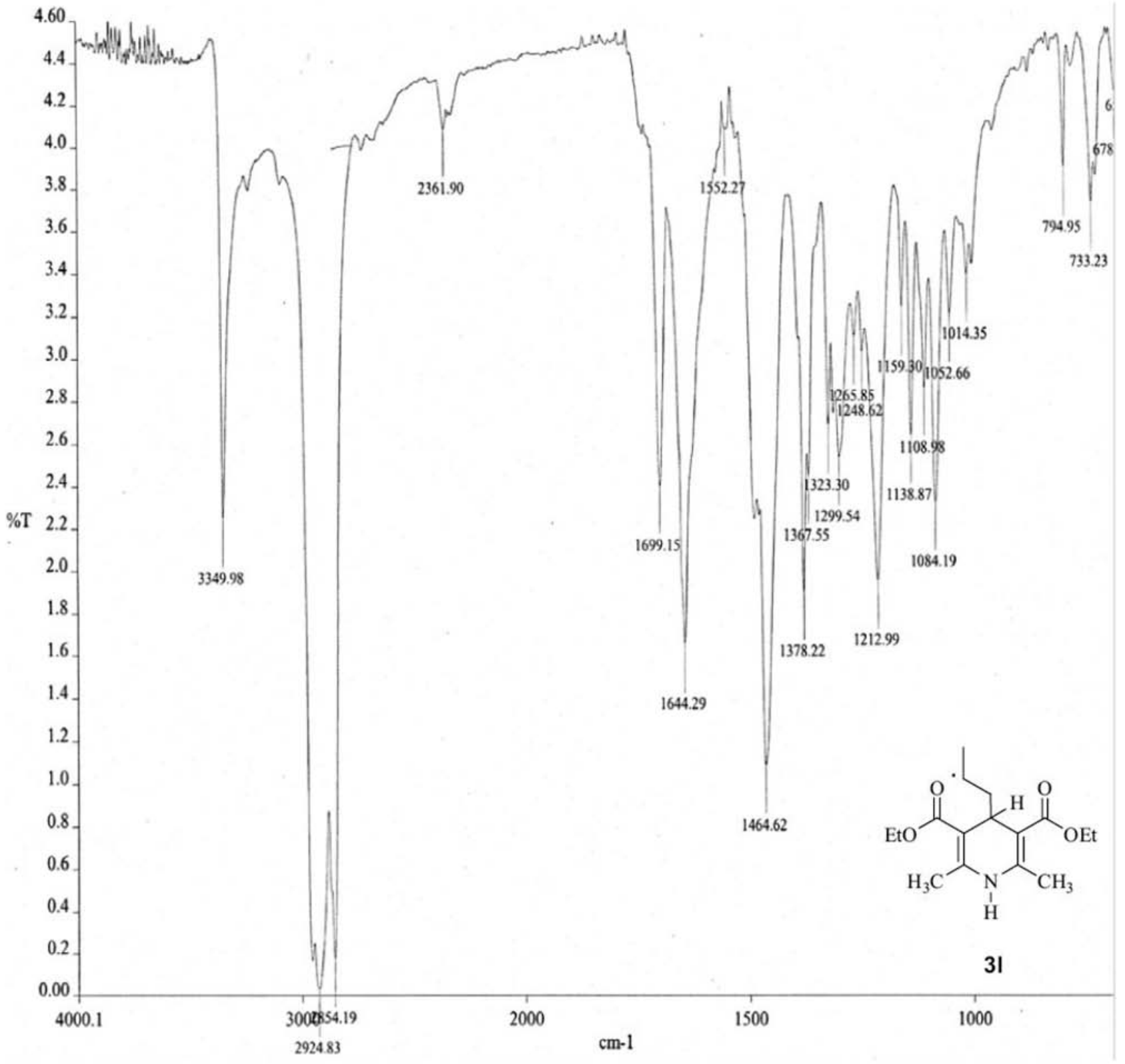

Figure S34. IR spectrum of 2,6-dimethyl-4-(n-propyl)-3,5-dicarboethoxy-1,4-dihydropyridine. 
<smiles>CCCC1(C(=O)OCC)C(C)=C(C)NC(C)=C1C(=O)OCC</smiles>

31

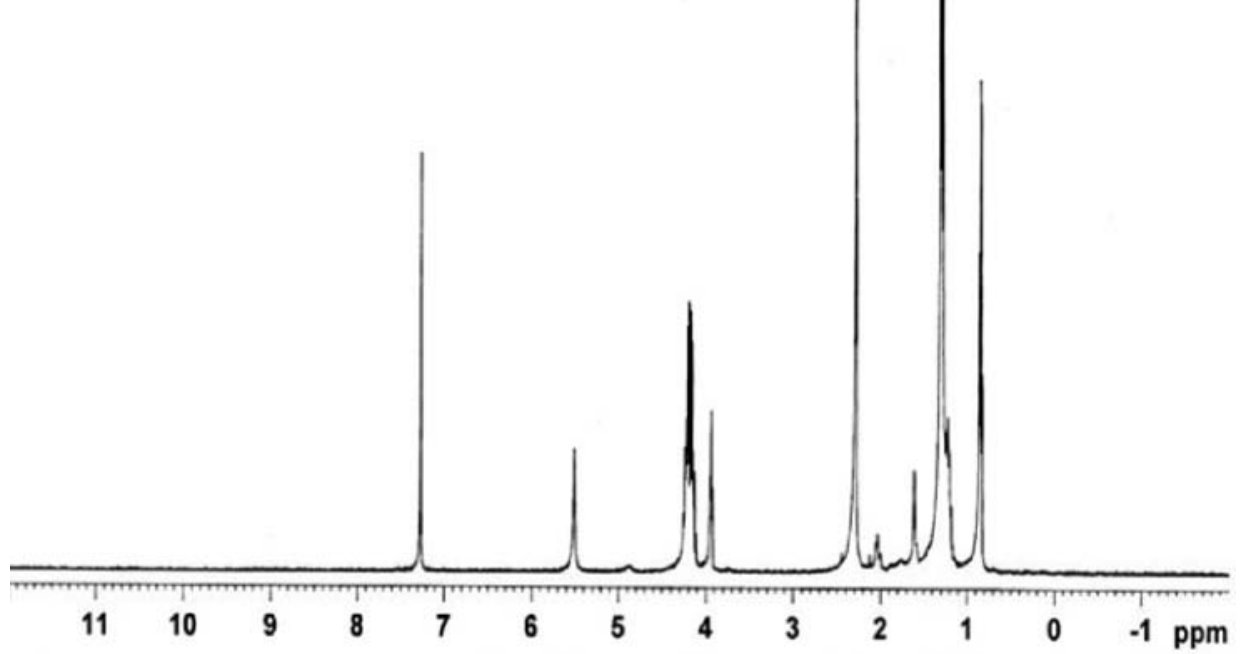

Figure S35. 'H NMR spectrum (400 MHz, $\mathrm{CDCl}_{3}$ ) of 2,6-dimethyl-4-(n-propyl)-3,5-dicarboethoxy-1,4-dihydropyridine.<smiles>CCCC1C(C(=O)OCC)=C(C)NC(C)=C1C(=O)OCC</smiles>

31

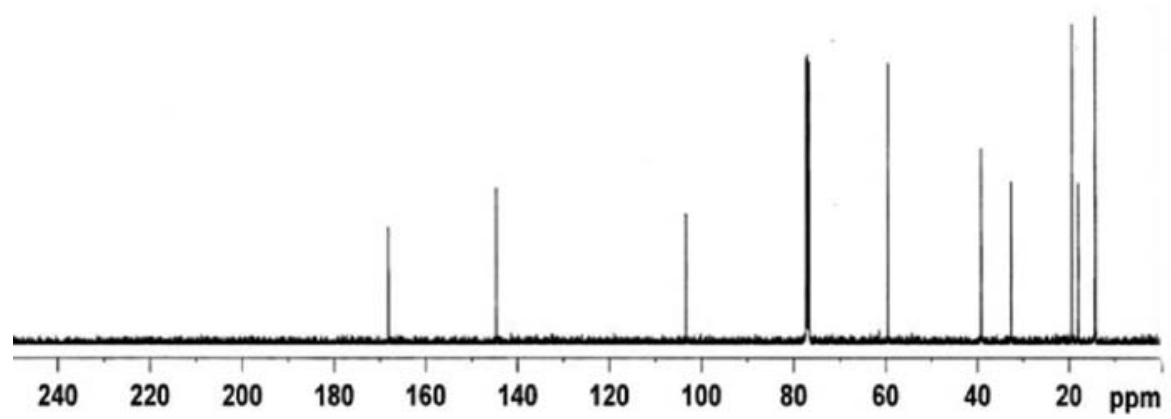

Figure S36. ${ }^{13} \mathrm{C}$ NMR spectrum (100 MHz, $\mathrm{CDCl}_{3}$ ) of 2,6-dimethyl-4-(n-propyl)-3,5-dicarboethoxy-1,4-dihydropyridine. 


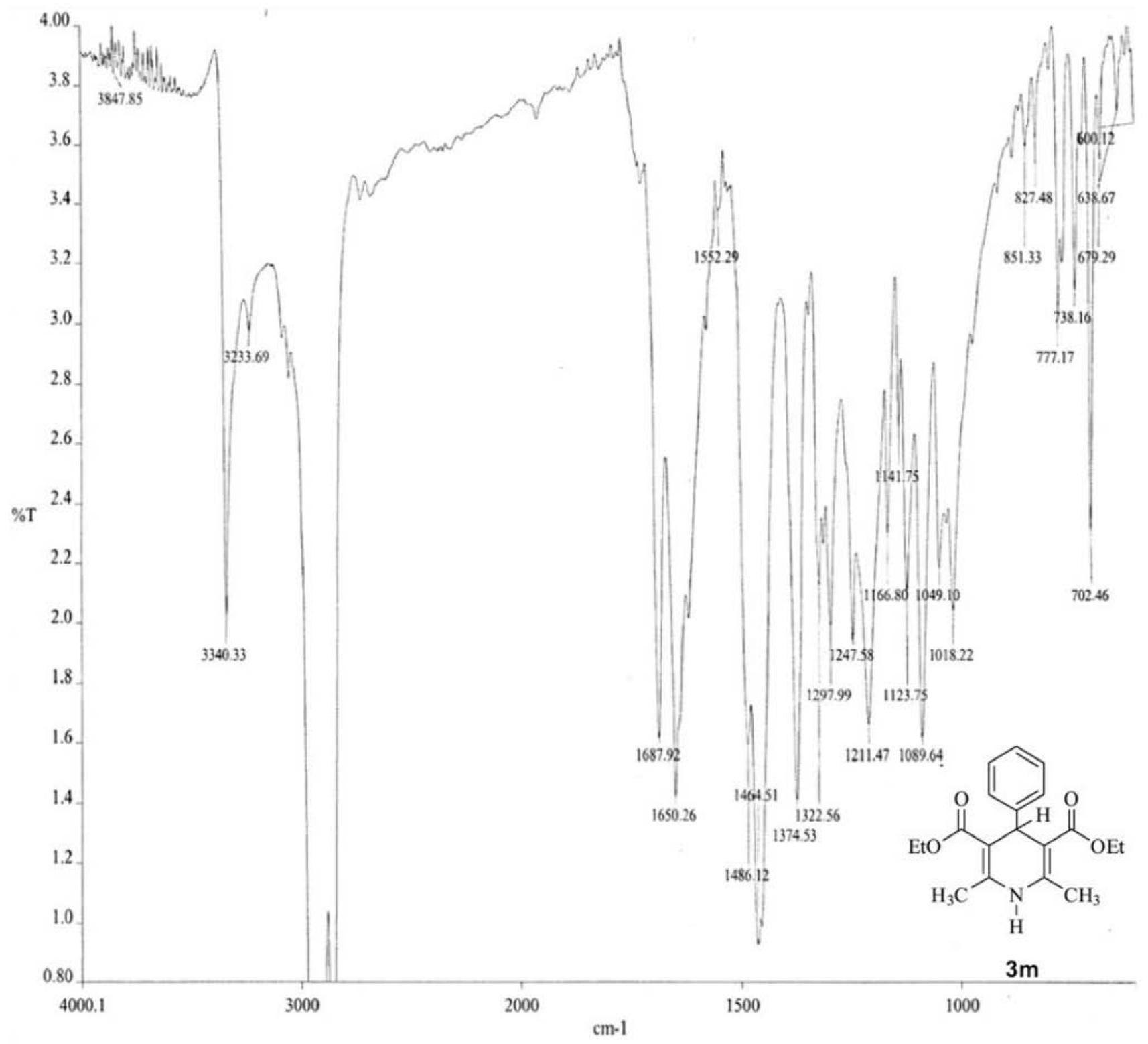

Figure S37. IR spectrum of 2,6-dimethyl-4-(phenyl)-3,5-dicarboethoxy-1,4-dihydropyridine. 
<smiles>CCOC(=O)C1=C(C)NC(C)=C(C(=O)OCC)C1(c1ccccc1)c1ccccc1</smiles>

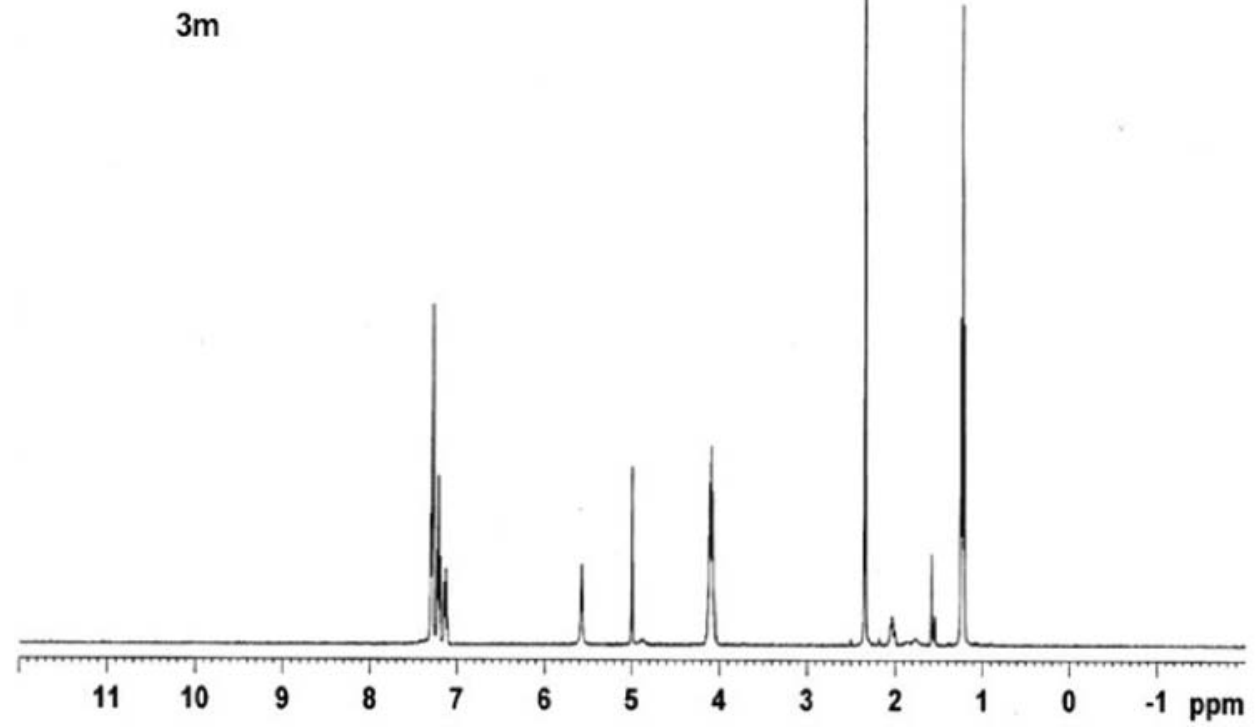

Figure S38. ${ }^{1} \mathrm{H}$ NMR spectrum (400 MHz, $\mathrm{CDCl}_{3}$ ) of 2,6-dimethyl-4-(phenyl)-3,5-dicarboethoxy-1,4-dihydropyridine.<smiles>CCOC(=O)C1=C(C)NC(C)=C(C(=O)OCC)C1(c1ccccc1)c1ccccc1</smiles>

$3 m$

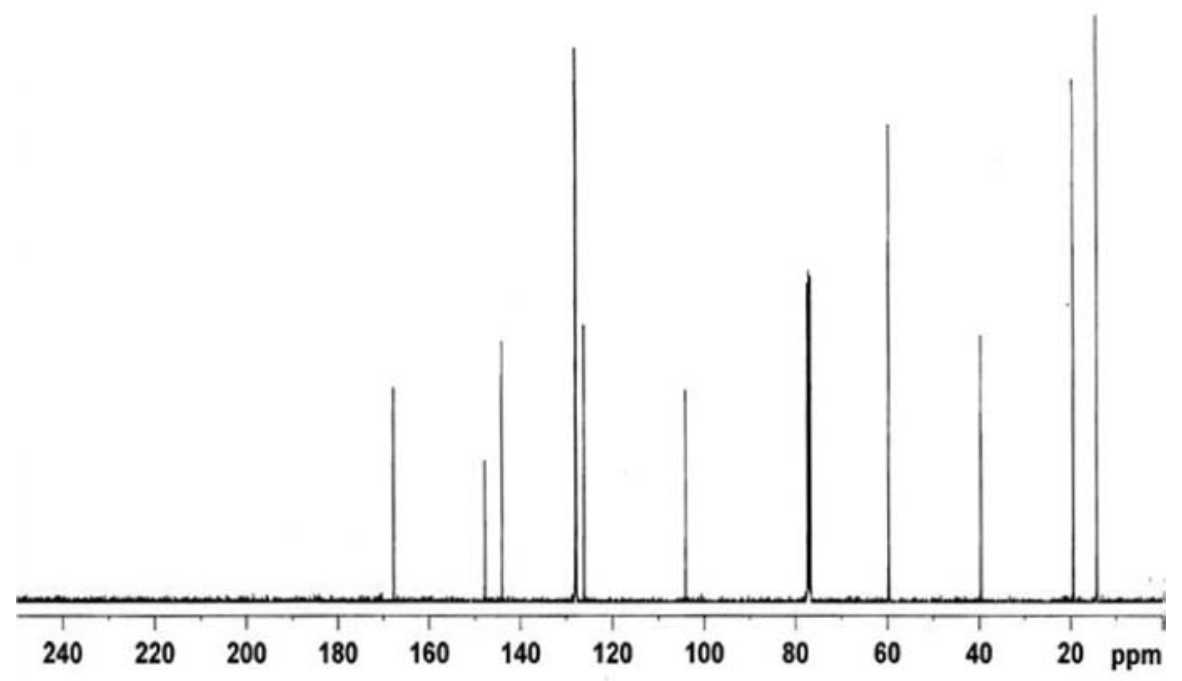

Figure S39. ${ }^{13} \mathrm{C}$ NMR spectrum (100 MHz, $\mathrm{CDCl}_{3}$ ) of 2,6-dimethyl-4-(phenyl)-3,5-dicarboethoxy-1,4-dihydropyridine. 


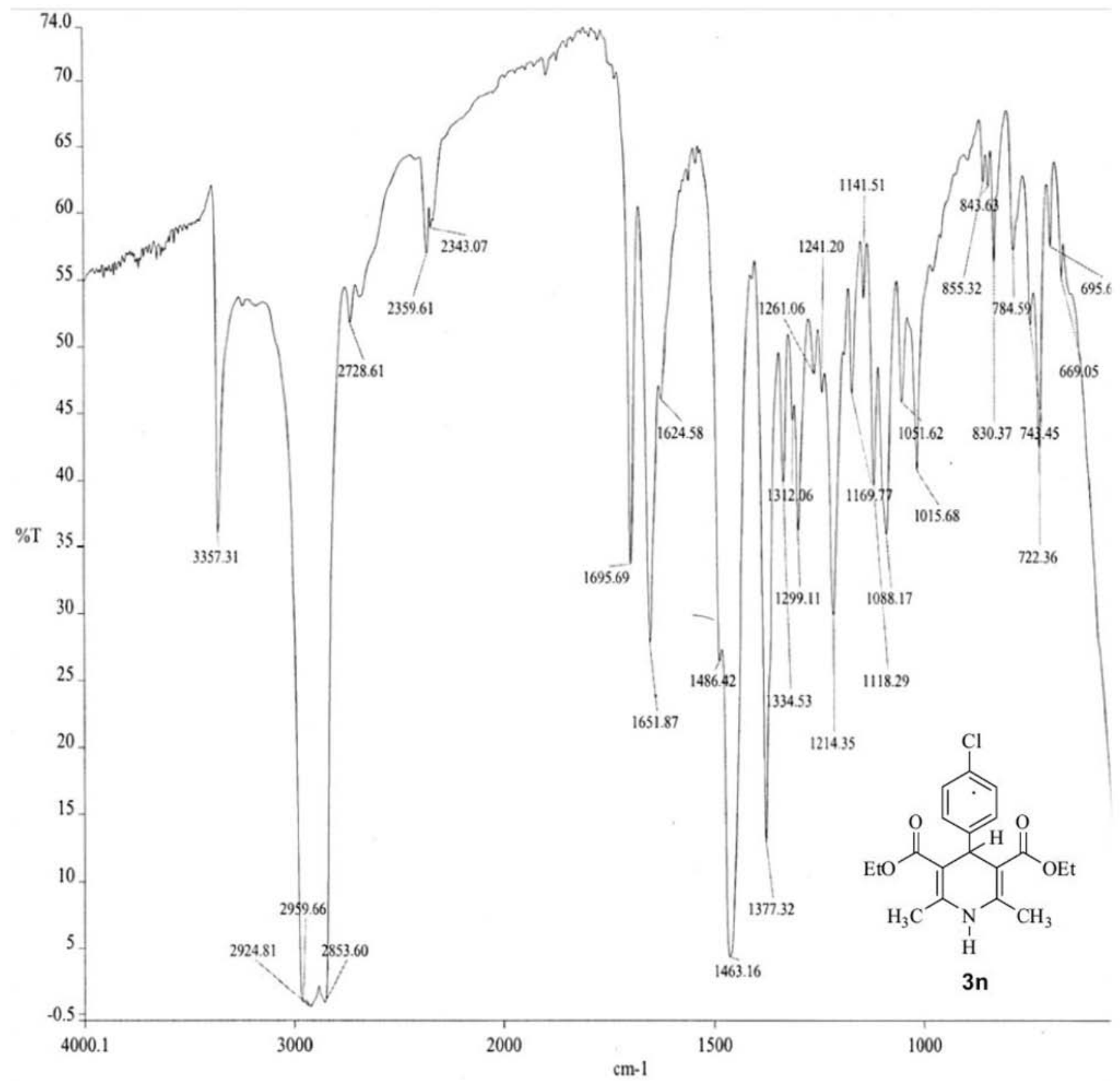

Figure S40. IR spectrum of 2,6-dimethyl-4-(4-chlorophenyl)-3,5-dicarboethoxy-1,4-dihydropyridine. 
<smiles>CCOC(=O)C1=C(C)NC(C)=C(C(=O)OCC)C1(c1ccccc1)c1ccc(Cl)cc1</smiles>

$3 n$

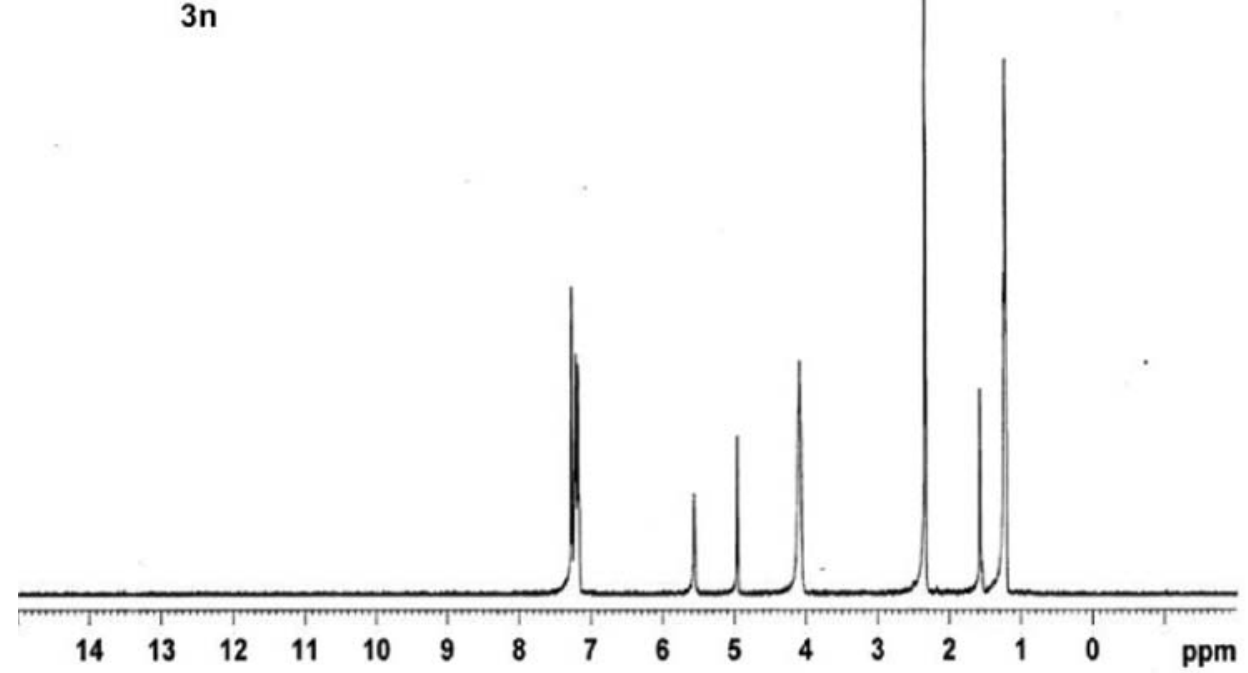

Figure S41. ${ }^{1} \mathrm{H}$ NMR spectrum (400 MHz, $\mathrm{CDCl}_{3}$ ) of 2,6-dimethyl-4-(4-chlorophenyl)-3,5-dicarboethoxy-1,4-dihydropyridine.<smiles>CCOC(=O)C1=C(C)N(Cc2ccccc2)C(C)=C(C(=O)OCC)C1c1ccc(Cl)cc1</smiles>

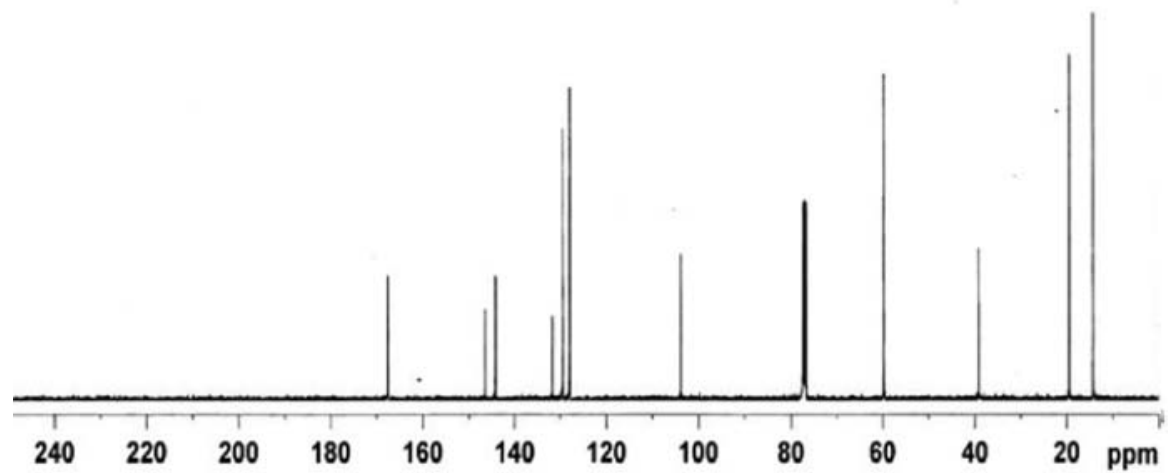

Figure S42. ${ }^{13} \mathrm{C}$ NMR spectrum (100 MHz, $\mathrm{CDCl}_{3}$ ) of 2,6-dimethyl-4-(4-chlorophenyl)-3,5-dicarboethoxy-1,4-dihydropyridine. 


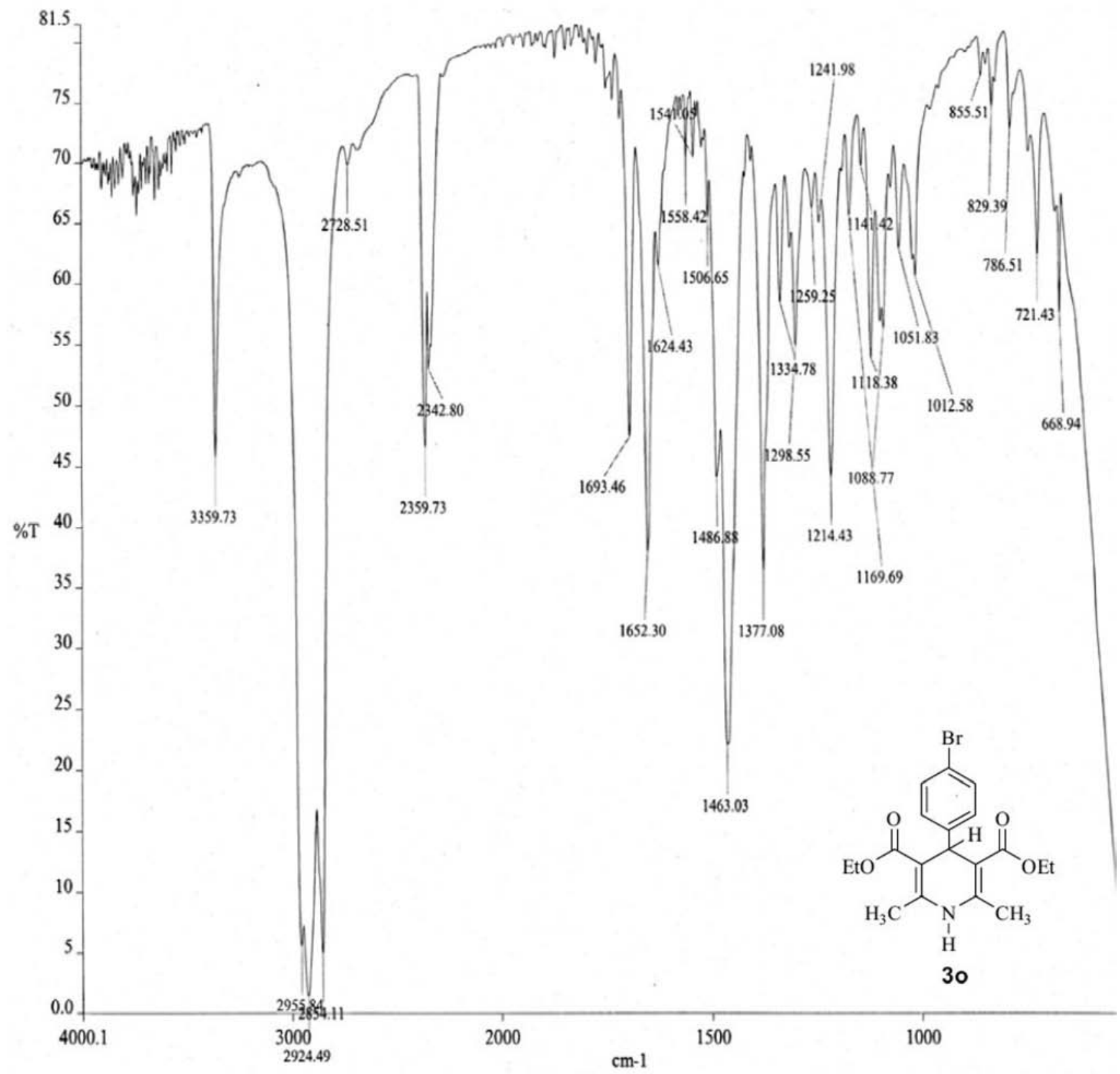

Figure S43. IR spectrum of 2,6-dimethyl-4-(4-bromophenyl)-3,5-dicarboethoxy-1,4-dihydropyridine. 
<smiles>CCOC(=O)C1=C(C)N(C=O)C(C)=C(C(=O)OCC)C1c1ccc(Br)cc1</smiles>
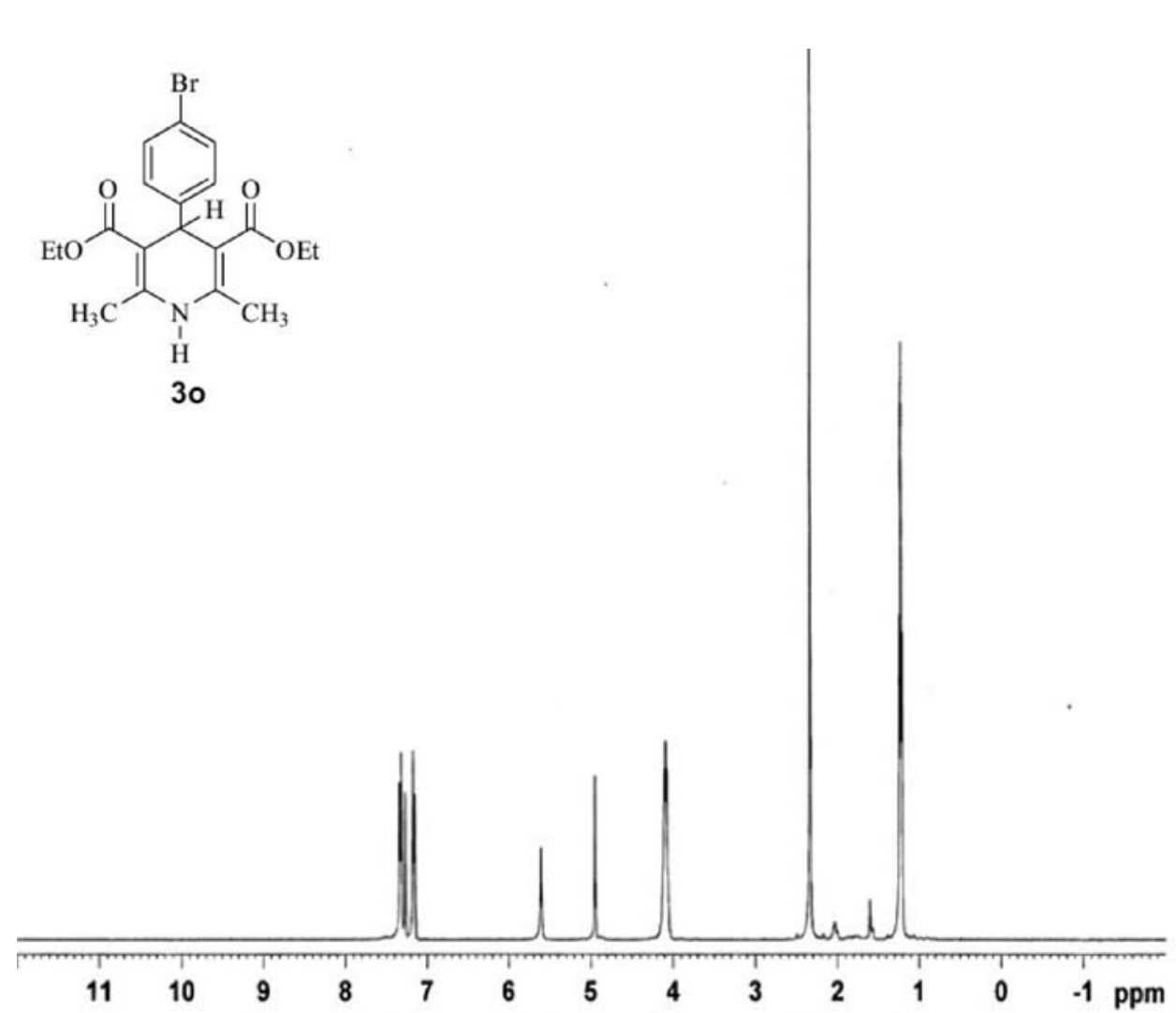

Figure S44. ${ }^{1} \mathrm{H}$ NMR spectrum (400 MHz, $\mathrm{CDCl}_{3}$ ) of 2,6-dimethyl-4-(4-bromophenyl)-3,5-dicarboethoxy-1,4-dihydropyridine.<smiles>C=CN1C(C)=C(C(=O)OCC)C(c2ccccc2)(c2ccc(Br)cc2)C(C(=O)OCC)=C1C</smiles>

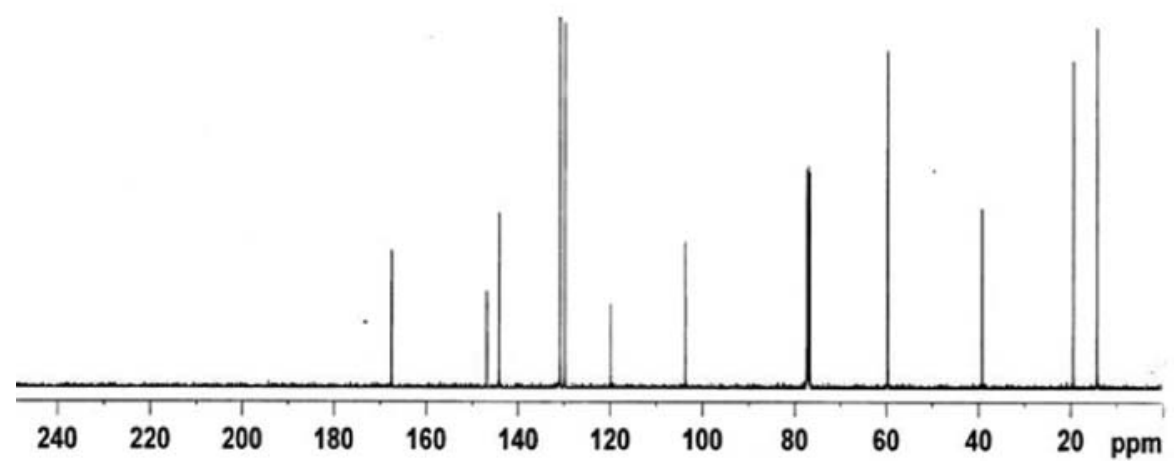

Figure S45. ${ }^{13} \mathrm{C}$ NMR spectrum (100 MHz, $\mathrm{CDCl}_{3}$ ) of 2,6-dimethyl-4-(4-bromophenyl)-3,5-dicarboethoxy-1,4-dihydropyridine. 


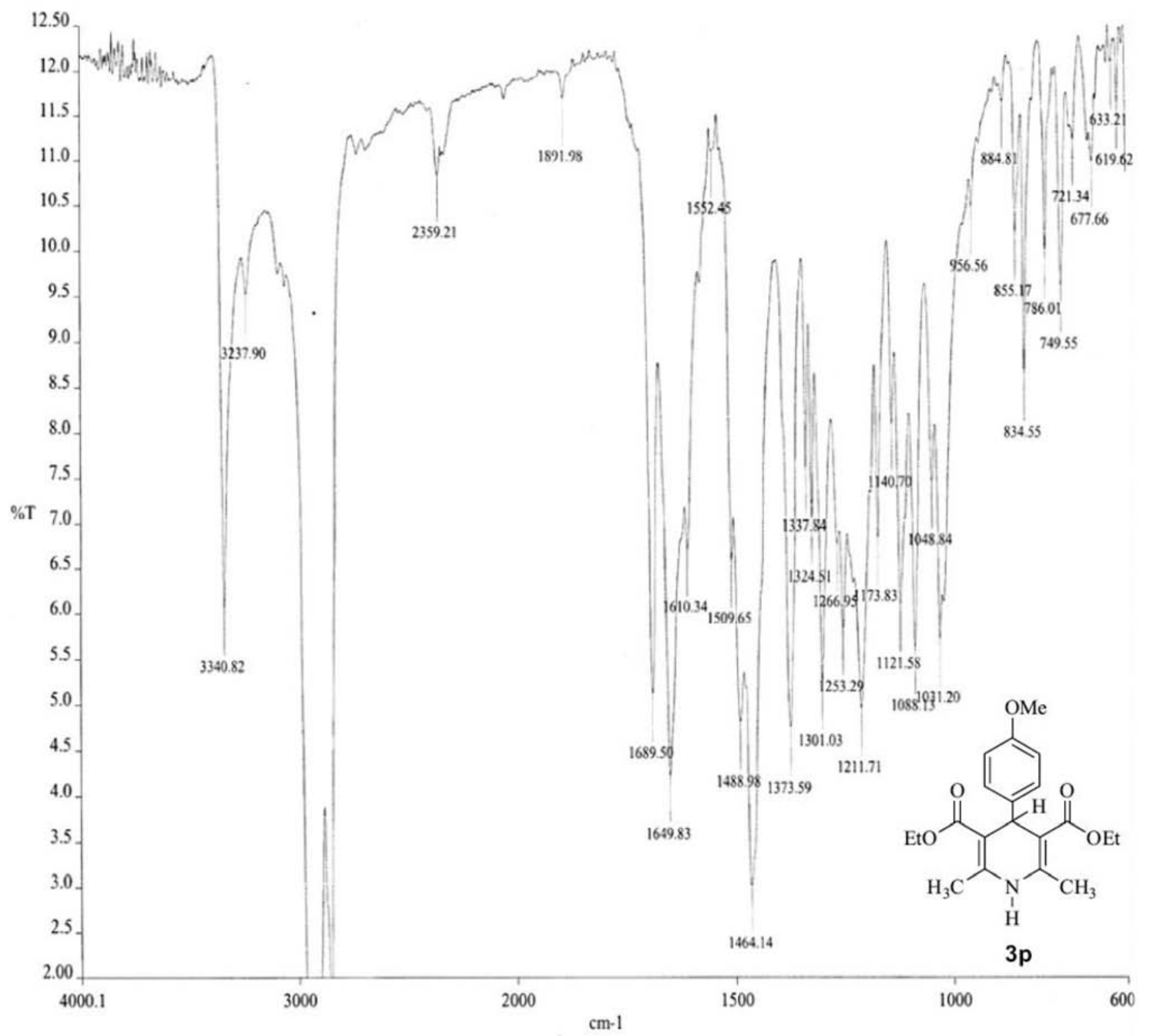

Figure S46. IR spectrum of 2,6-dimethyl-4-(4-methoxyphenyl)-3,5-dicarboethoxy-1,4-dihydropyridine. 
<smiles>CCOC(=O)C1=C(C)N(NC(=O)c2ccccc2)C(C(=O)OCC)(c2ccc(OC)cc2)C(C)=C1C</smiles>

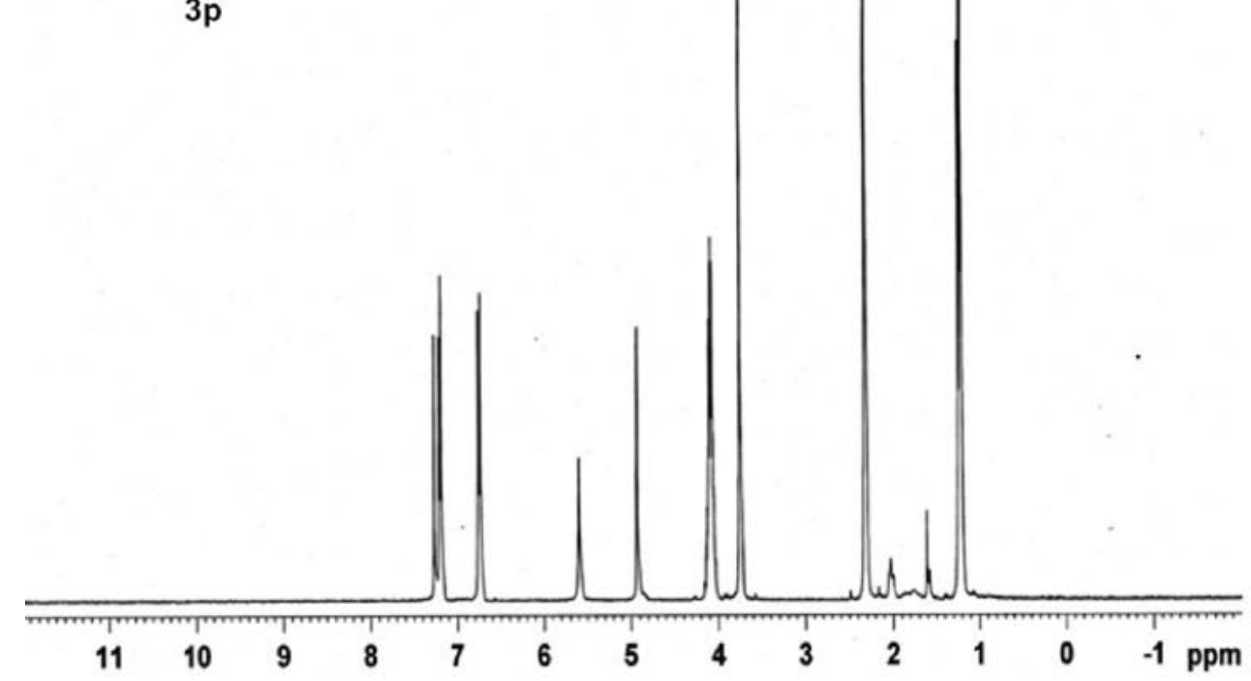

Figure S47. ${ }^{1} \mathrm{H}$ NMR spectrum (400 MHz, $\mathrm{CDCl}_{3}$ ) of 2,6-dimethyl-4-(4-methoxyphenyl)-3,5-dicarboethoxy-1,4-dihydropyridine.<smiles></smiles>

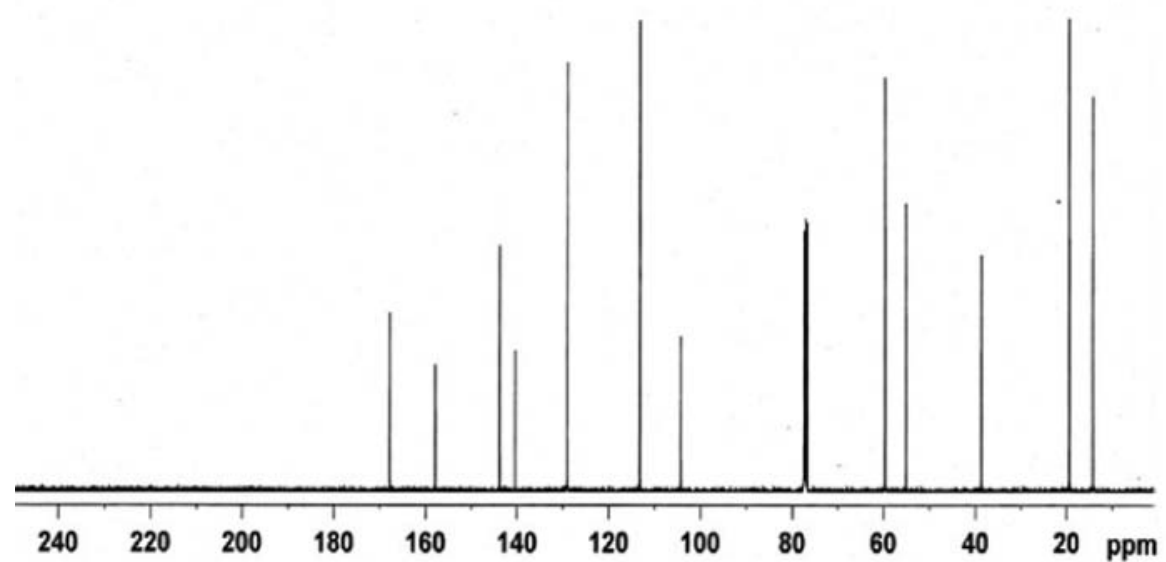

Figure S48. ${ }^{13} \mathrm{C}$ NMR spectrum (100 MHz, $\mathrm{CDCl}_{3}$ ) of 2,6-dimethyl-4-(4-methoxyphenyl)-3,5-dicarboethoxy-1,4-dihydropyridine. 


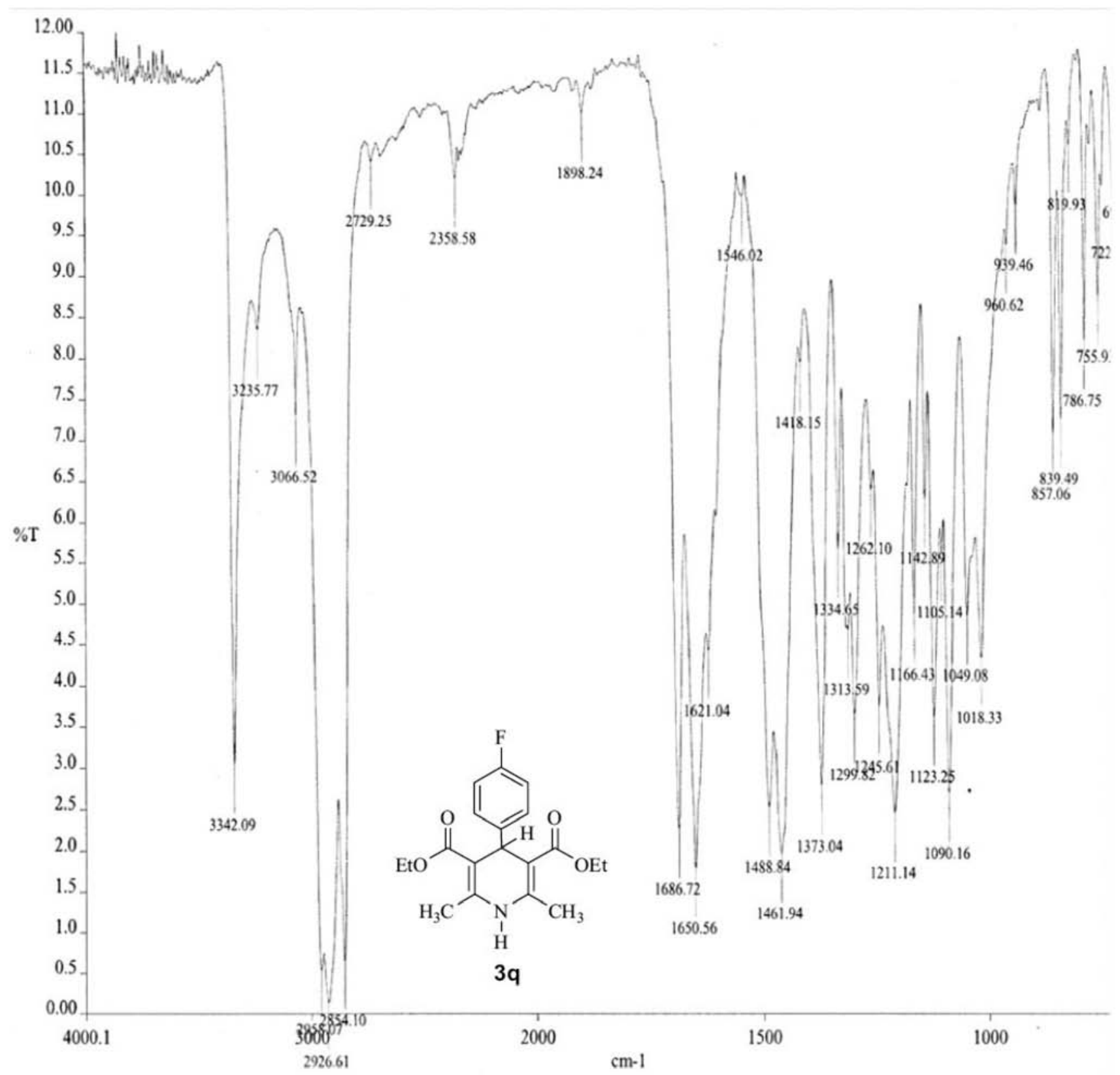

Figure S49. IR spectrum of 2,6-dimethyl-4-(4-fluorophenyl)-3,5-dicarboethoxy-1,4-dihydropyridine. 
<smiles>CCOC(=O)C1=C(C)N([13CH3])C(C)=C(C(=O)OCC)C1(c1ccccc1)c1ccc(F)cc1</smiles>

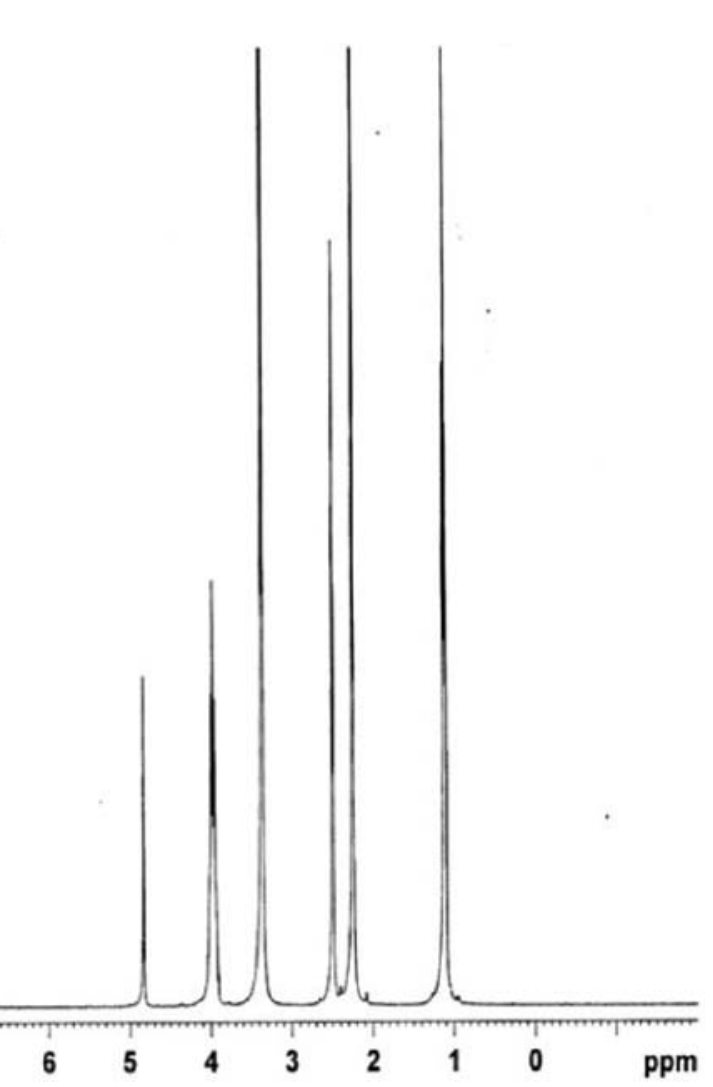

Figure S50. ${ }^{1} \mathrm{H}$ NMR spectrum (400 MHz, DMSO- $d_{6}$ ) of 2,6-dimethyl-4-(4-fluorophenyl)-3,5-dicarboethoxy-1,4-dihydropyridine.<smiles>CCCCCN1C(C)=C(C(=O)OCC)C(c2ccc(F)cc2)C(C(=O)OCC)=C1C</smiles>

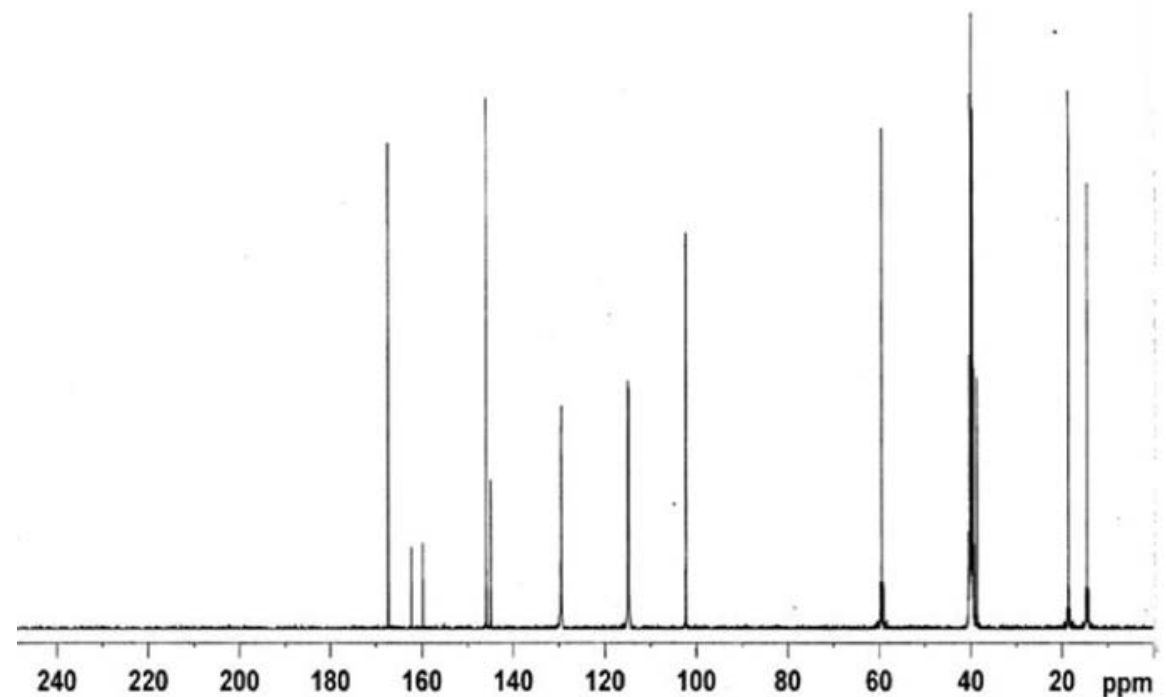

Figure S51. ${ }^{13} \mathrm{C}$ NMR spectrum (100 MHz, DMSO- $d_{6}$ ) of 2,6-dimethyl-4-(4-fluorophenyl)-3,5-dicarboethoxy-1,4-dihydropyridine. 


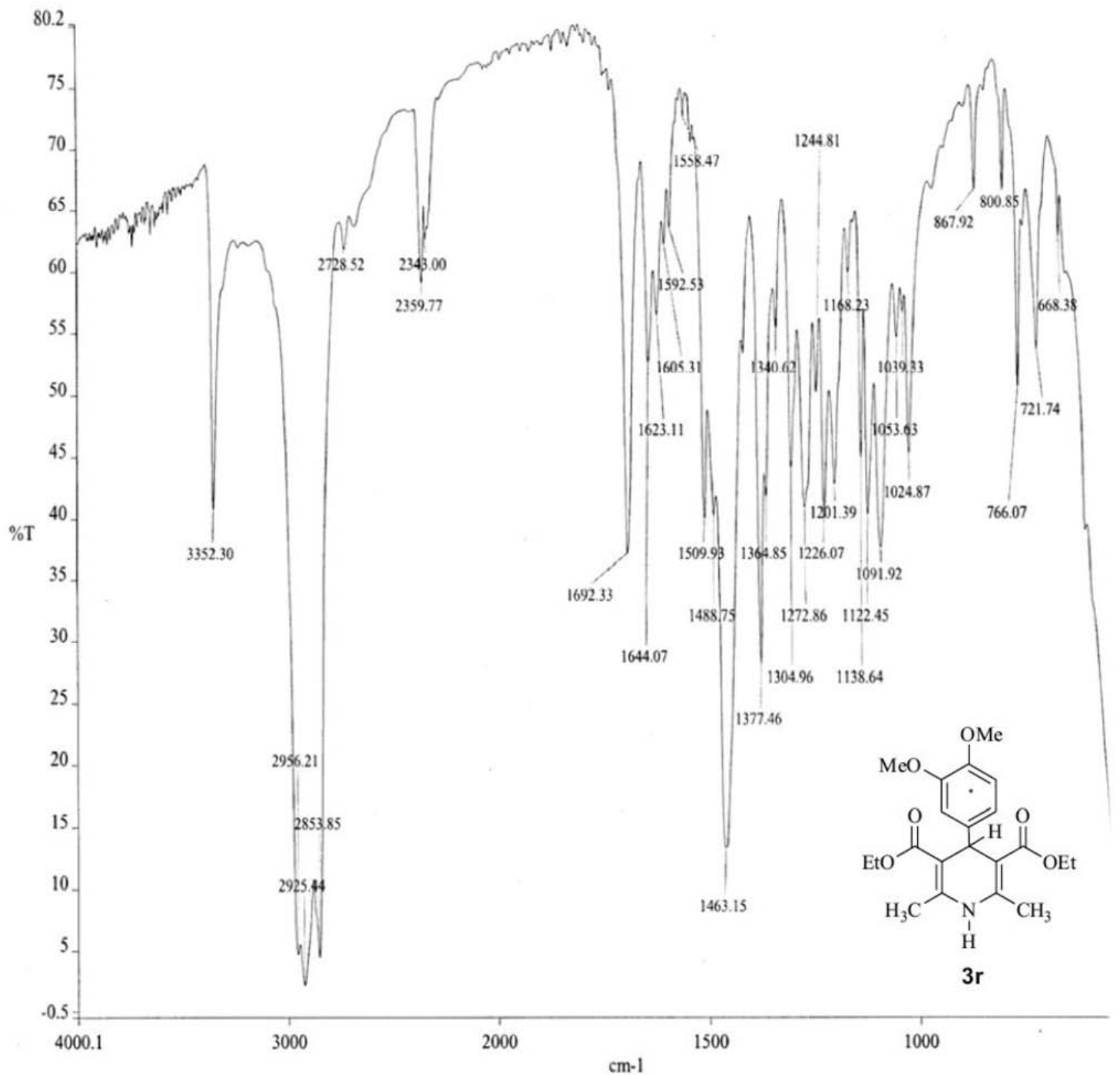

Figure S52. IR spectrum of 2,6-dimethyl-4-(3,4-dimethoxyphenyl)-3,5-dicarboethoxy-1,4-dihydropyridine. 
<smiles>CCOC(=O)C1=C(C)N(Br)C(C)=C(C(=O)OCC)C1c1ccc(OC)c(OC)c1</smiles>

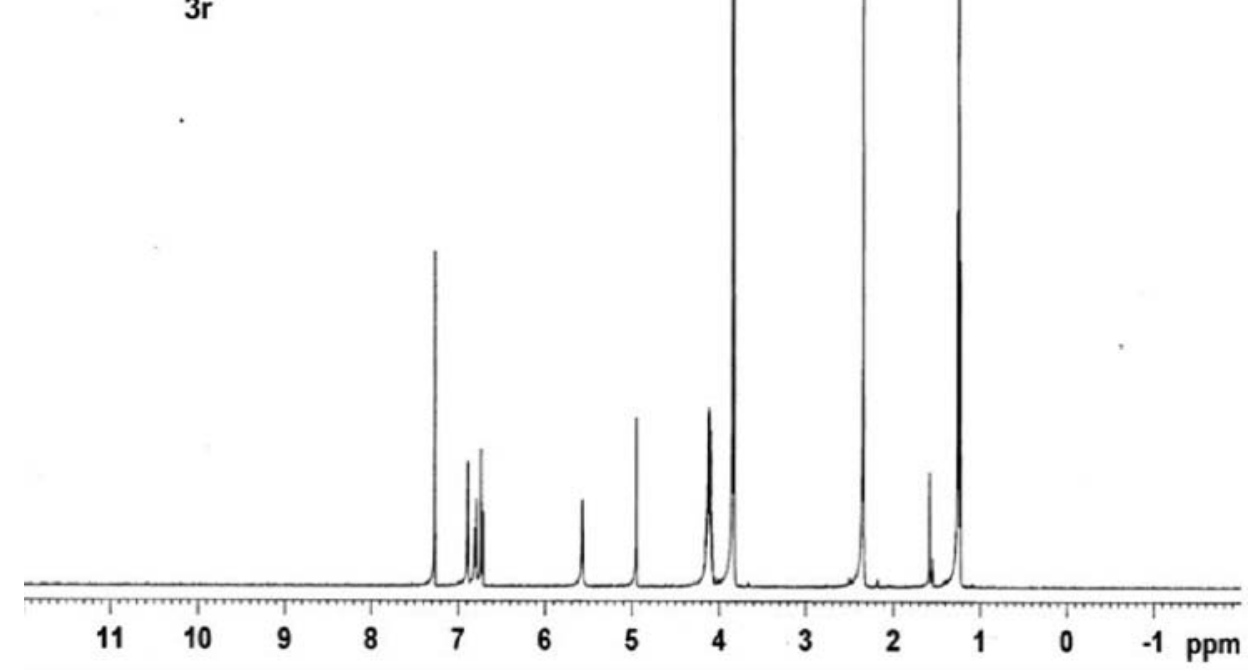

Figure S53. ${ }^{~} \mathrm{H}$ NMR spectrum (400 MHz, $\mathrm{CDCl}_{3}$ ) of 2,6-dimethyl-4-(3,4-dimethoxyphenyl)-3,5-dicarboethoxy-1,4-dihydropyridine.<smiles>CCOC(=O)C1=C(C)N(C)C(C)=C(C(=O)OCC)C1c1ccc(OC)c(OC)c1</smiles>

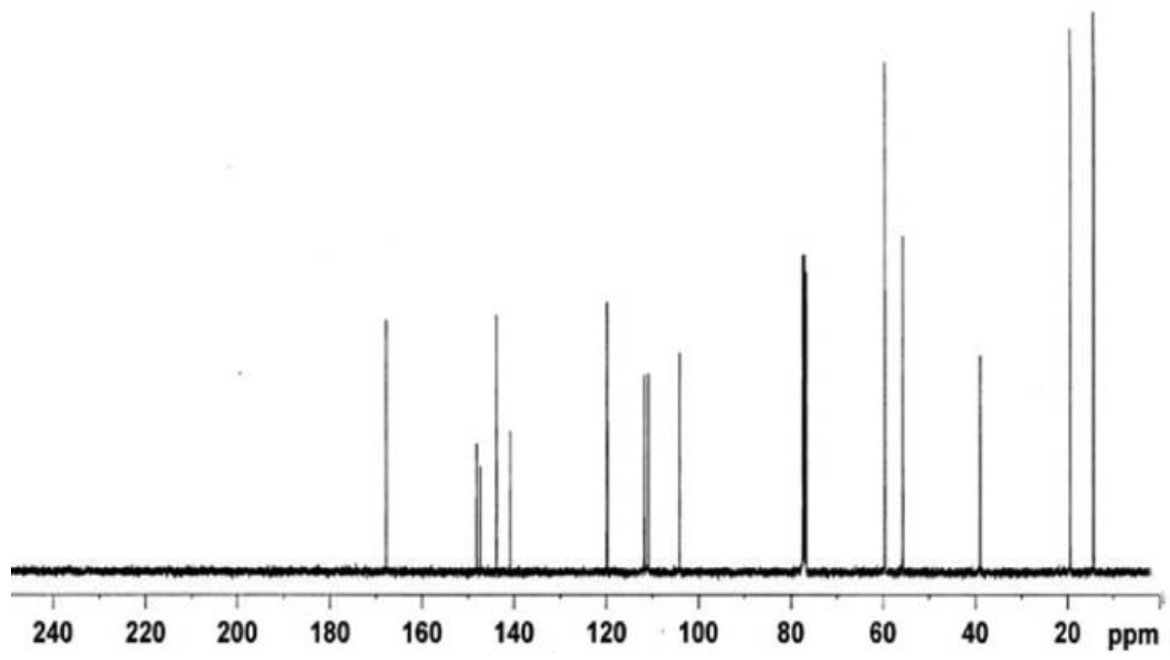

Figure S54. ${ }^{13} \mathrm{C}$ NMR spectrum (100 MHz, $\mathrm{CDCl}_{3}$ ) of 2,6-dimethyl-4-(3,4-dimethoxyphenyl)-3,5-dicarboethoxy-1,4-dihydropyridine. 


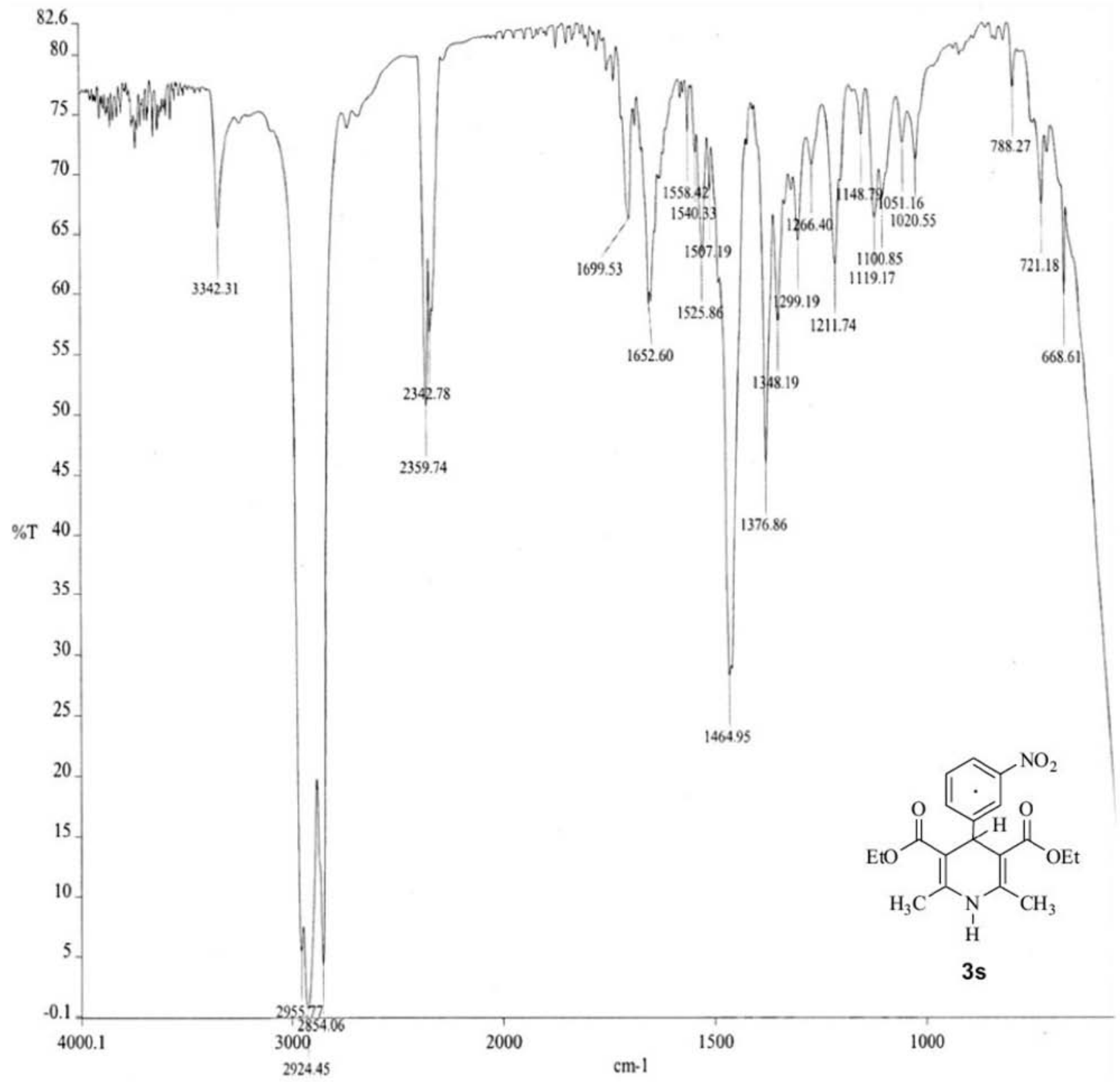

Figure S55. IR spectrum of 2,6-dimethyl-4-(3-nitrophenyl)-3,5-dicarboethoxy-1,4-dihydropyridine. 
<smiles>CCOC(=O)C1=C(C)NC(C)=C(C(=O)OCC)C1c1cccc([N+](=O)[O-])c1</smiles>

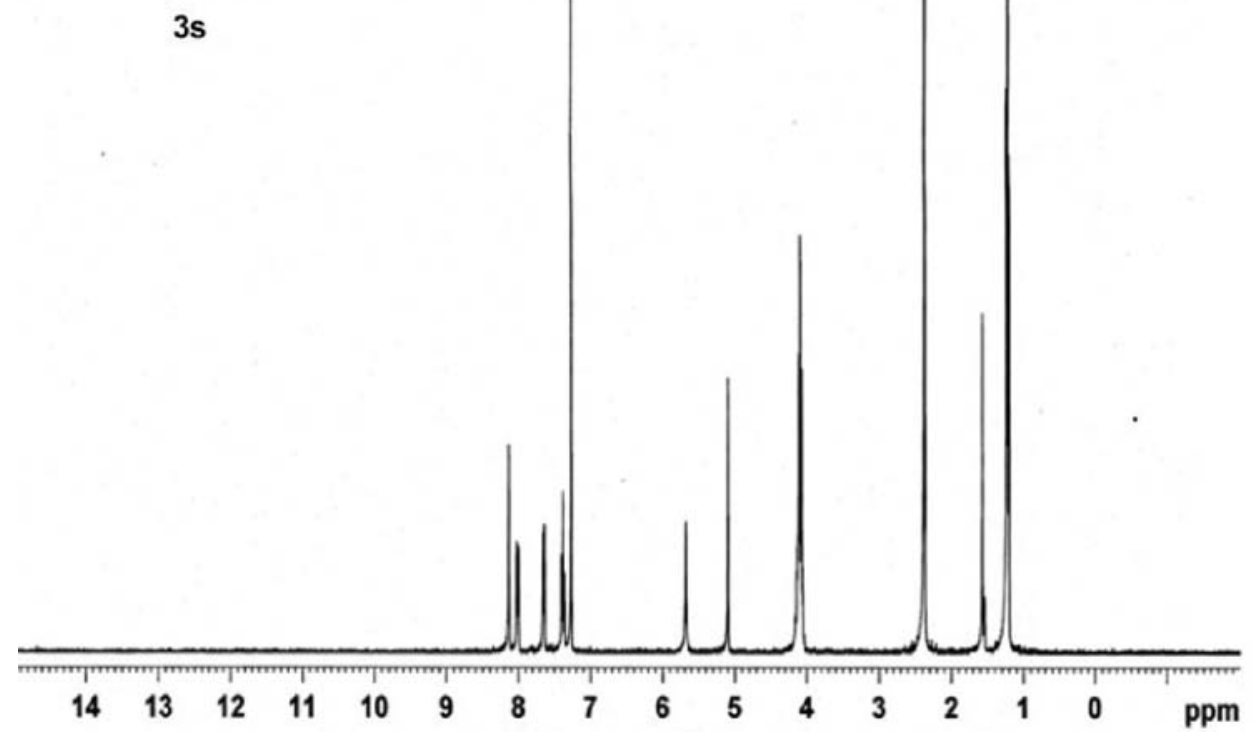

Figure S56. ${ }^{1} \mathrm{H}$ NMR spectrum (400 MHz, $\mathrm{CDCl}_{3}$ ) of 2,6-dimethyl-4-(3-nitrophenyl)-3,5-dicarboethoxy-1,4-dihydropyridine.<smiles>CCOC(=O)C1=C(C)NC(C)=C(C(=O)OCC)C1(C(=O)OC)c1cccc([N+](=O)[O-])c1</smiles>

3s

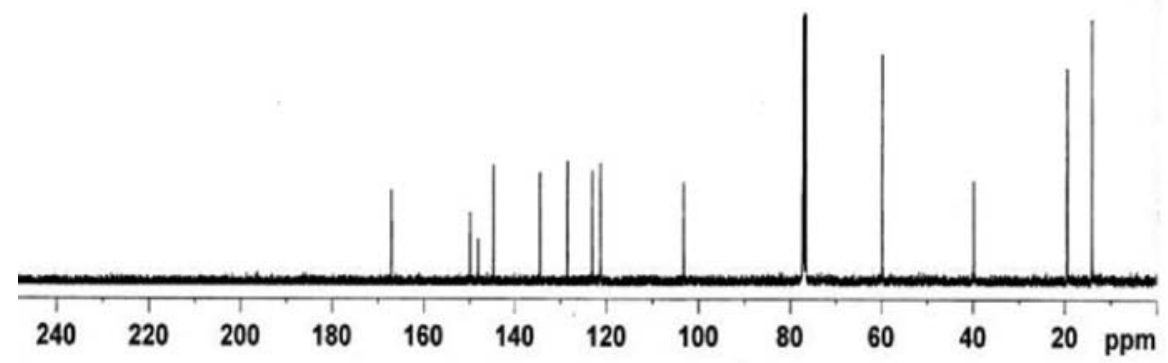

Figure S57. ${ }^{13} \mathrm{C}$ NMR spectrum (100 MHz, $\mathrm{CDCl}_{3}$ ) of 2,6-dimethyl-4-(3-nitrophenyl)-3,5-dicarboethoxy-1,4-dihydropyridine. 
<smiles>CCOC(=O)C1=C(C)NC(C)=C(C(=O)OCC)C1c1cccc(C2C(C(=O)OCC)=C(C)NC(C)=C2C(=O)OCC)c1</smiles>

3'a

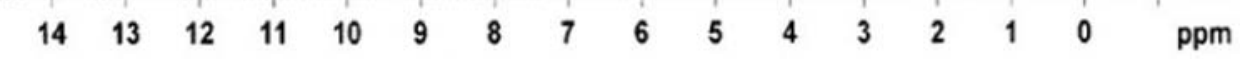

Figure S58. ${ }^{1} \mathrm{H}$ NMR spectrum (400 MHz, $\mathrm{CDCl}_{3}$ ) of 1,3-bis-(2,6-diethyl-3,5-dicarboethoxy-1,4-dihydropyridine) benzene.

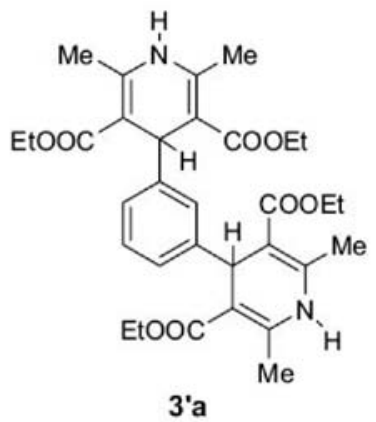

3'a

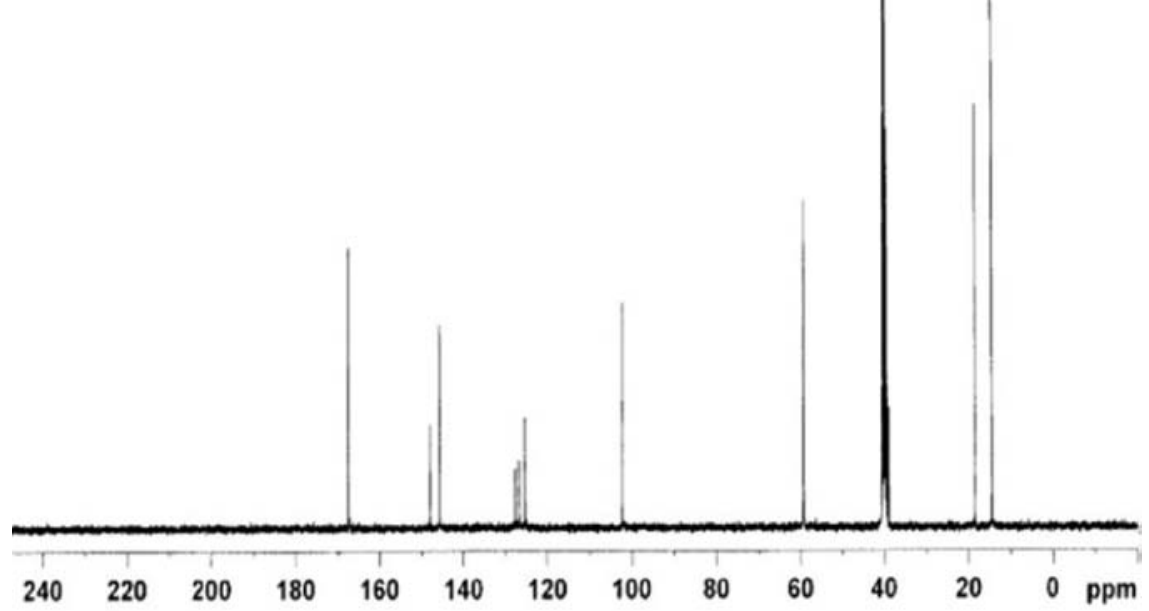

Figure S59. ${ }^{13} \mathrm{C}$ NMR spectrum (100 $\mathrm{MHz}, \mathrm{CDCl}_{3}$ ) of 1,3-bis-(2,6-diethyl-3,5-dicarboethoxy-1,4-dihydropyridine) benzene. 


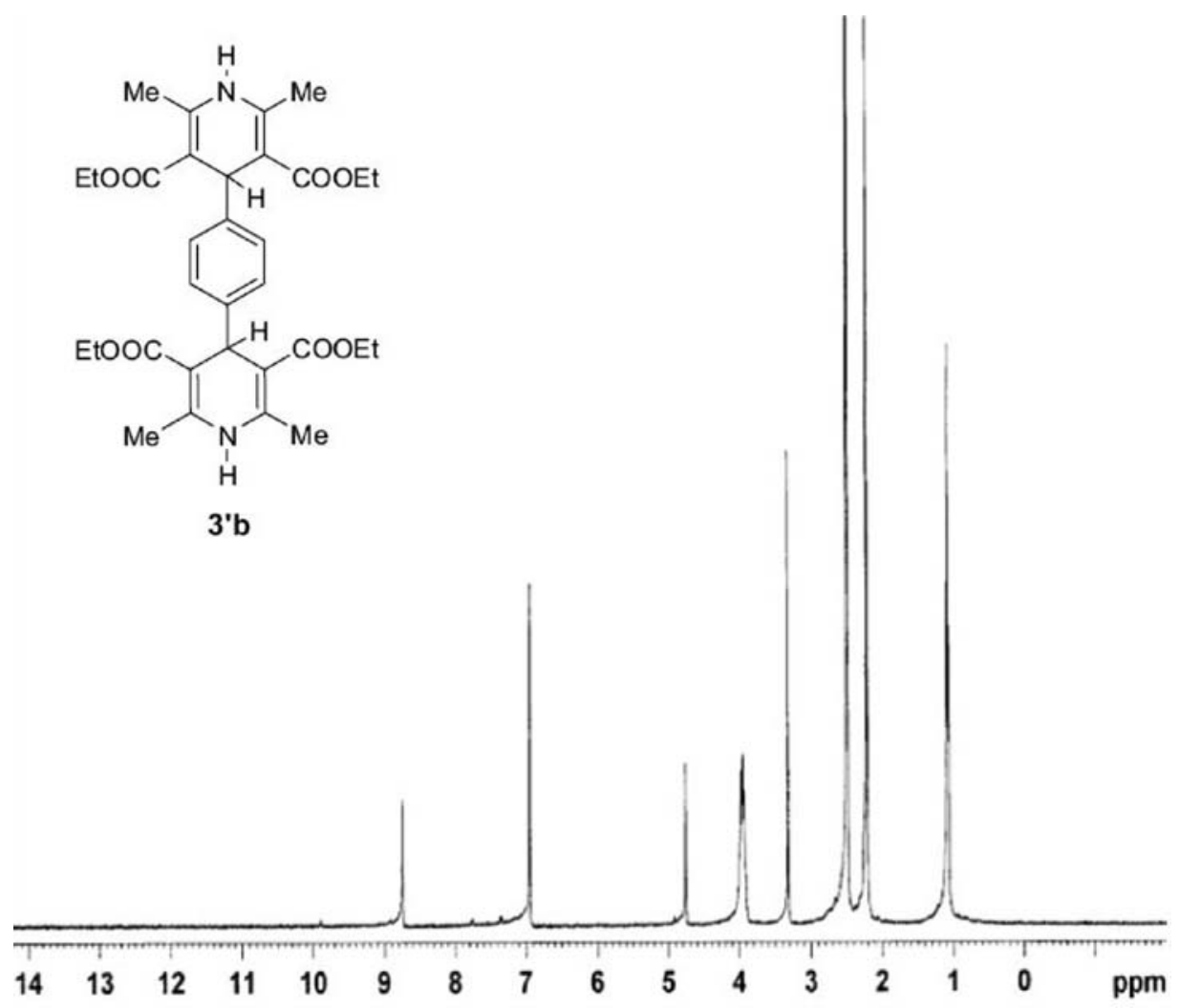

Figure S60. ${ }^{~} \mathrm{H}$ NMR spectrum (400 MHz, $\mathrm{CDCl}_{3}$ ) of 1,4-bis-(2,6-diethyl-3,5-dicarboethoxy-1,4-dihydropyridine) benzene.

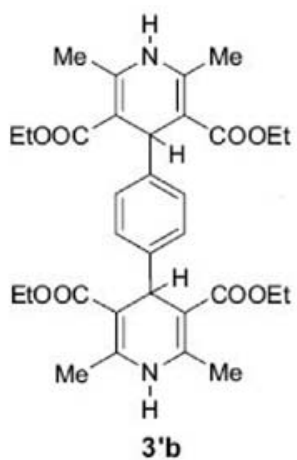

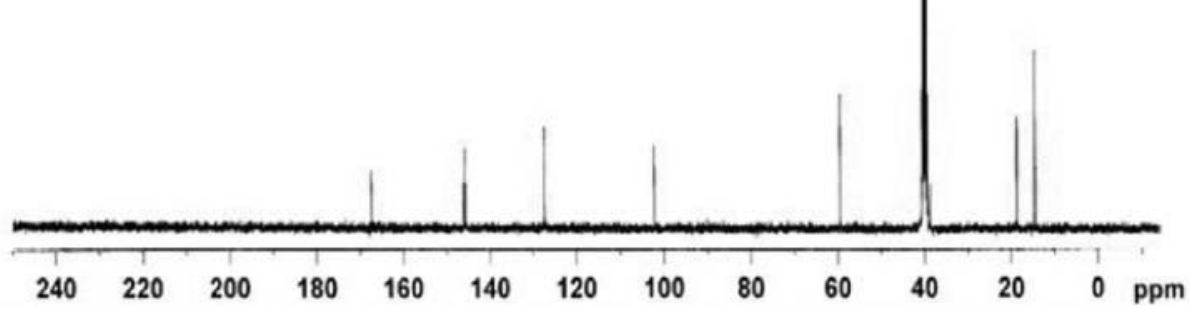

Figure S61. ${ }^{13} \mathrm{C}$ NMR spectrum (100 MHz, $\mathrm{CDCl}_{3}$ ) of 1,4-bis-(2,6-diethyl-3,5-dicarboethoxy-1,4-dihydropyridine) benzene. 
<smiles>CC(=O)C1=C(C(C)=O)C(c2cccc(C3C(C(C)=O)=C(C)NC(C)=C3C(C)=O)c2)C(C(C)=O)=C(C(C)=O)N1</smiles>

3'c

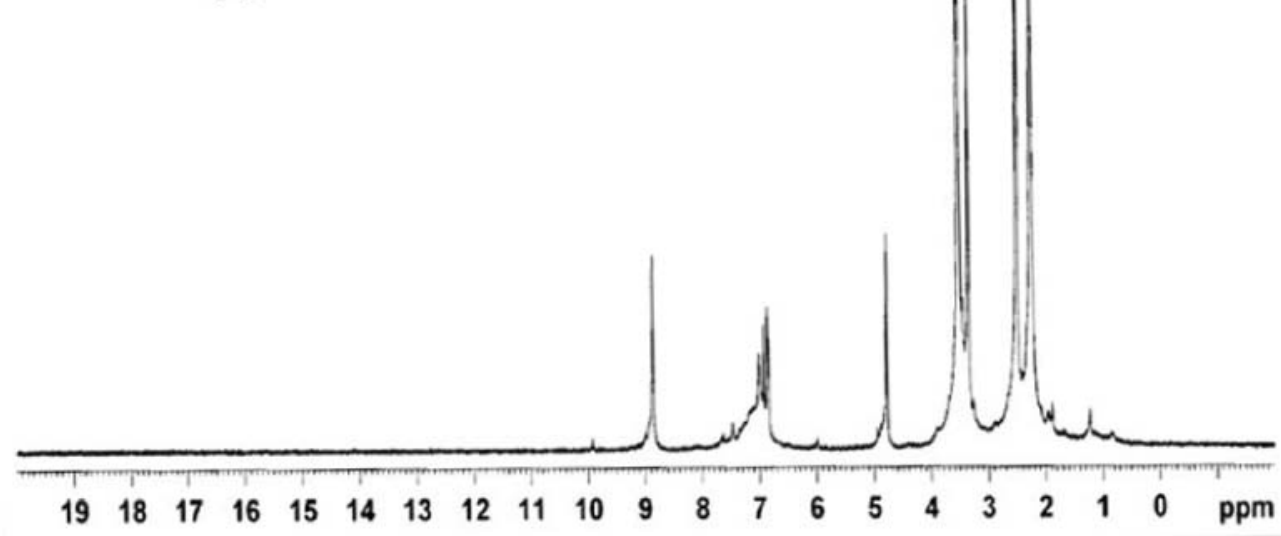

Figure S62. ${ }^{1} \mathrm{H}$ NMR spectrum (400 $\mathrm{MHz}, \mathrm{CDCl}_{3}$ ) of 1,3- bis-(2,6-dimethyl-3,5-dicarboethoxy-1,4-dihydropyridine) benzene.

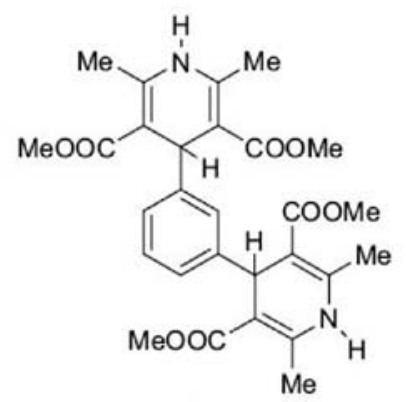

3'c

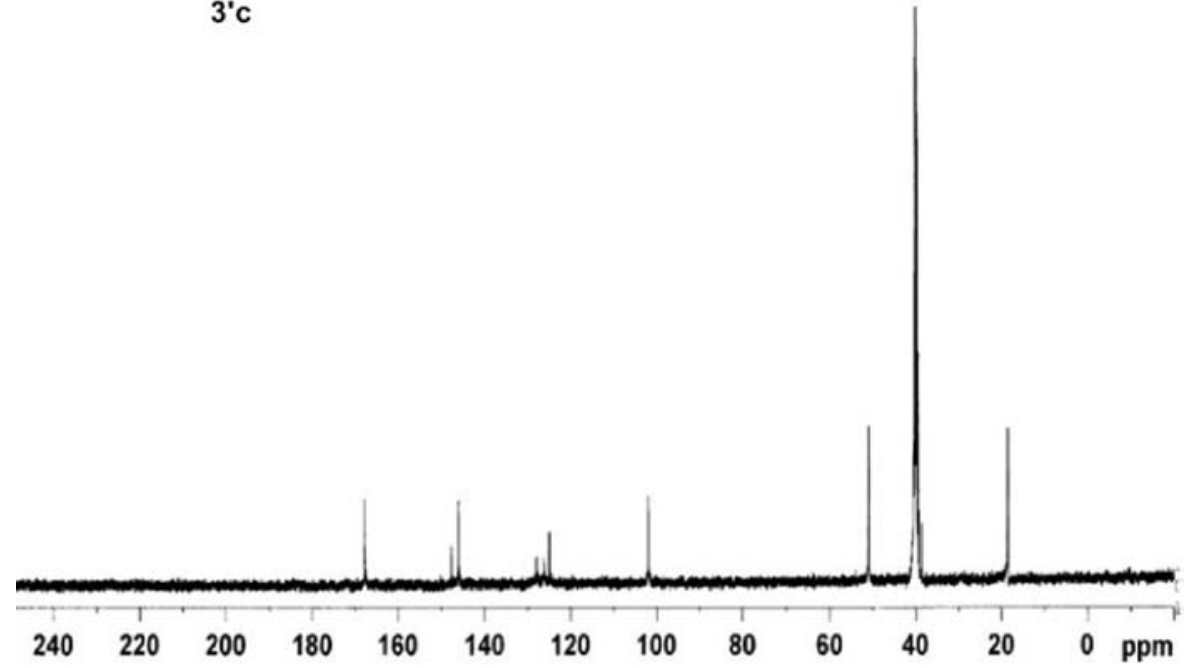

Figure S63. ${ }^{13} \mathrm{C}$ NMR spectrum (100 MHz, $\mathrm{CDCl}_{3}$ ) of 1,3-bis-(2,6-dimethyl-3,5-dicarboethoxy-1,4-dihydropyridine) benzene. 


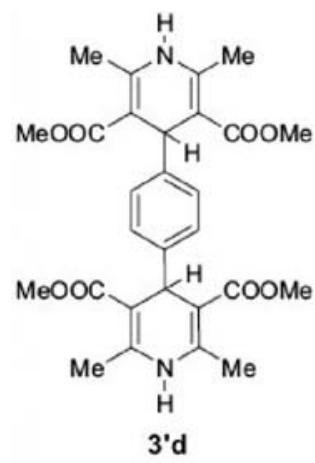

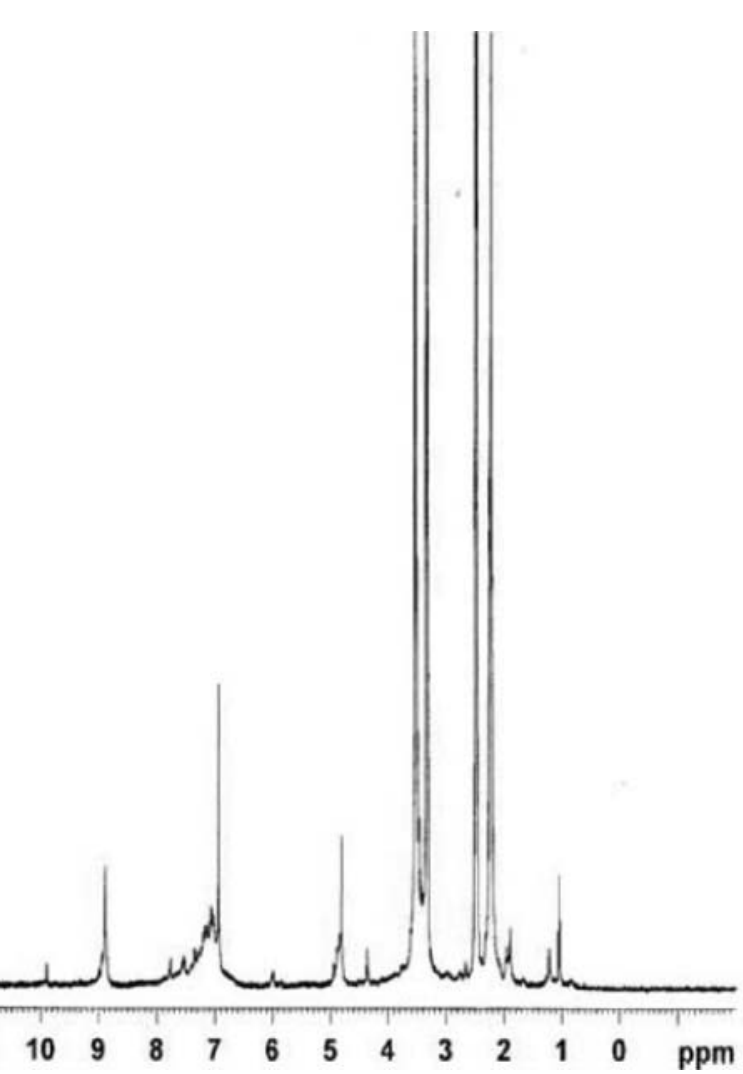

Figure S64. ${ }^{1} \mathrm{H}$ NMR spectrum (400 MHz, $\mathrm{CDCl}_{3}$ ) of 1,4-bis-(2,6-dimethyl-3,5-dicarboethoxy-1,4-dihydropyridine) benzene.<smiles>COC(=O)C1=C(C(=O)OC)C(c2ccc(C3C(C)=C(C(C)=O)NC([N+](=O)[O-])=C3C(=O)OC)cc2)C(C(=O)OC)=C(C)N1</smiles>

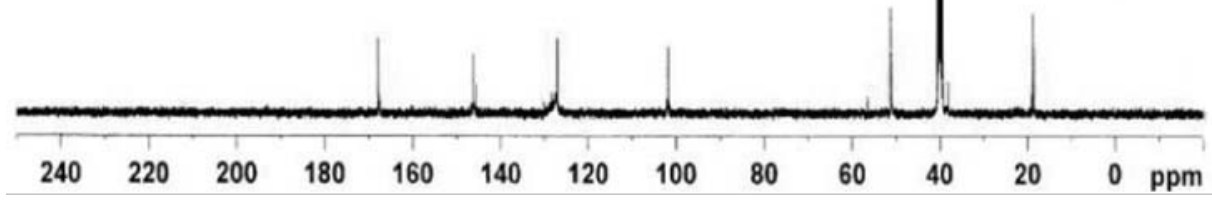

Figure S65. ${ }^{13} \mathrm{C}$ NMR spectrum (100 MHz, $\mathrm{CDCl}_{3}$ ) of 1,4-bis-(2,6-dimethyl-3,5-dicarboethoxy-1,4-dihydropyridine) benzene. 